Aus der Abteilung Psychosomatische Medizin und Psychotherapie (Prof. Dr. med. C. Herrmann-Lingen) im Zentrum Psychosoziale Medizin der Medizinischen Fakultät der Universität Göttingen

\title{
Der Einfluss psychischer Faktoren auf die Prognose nach perkutaner Koronarintervention
}

\author{
INAUGURAL - DISSERTATION \\ zur Erlangung des Doktorgrades \\ der Medizinischen Fakultät \\ der Georg-August-Universität zu Göttingen \\ vorgelegt von \\ Sharif Hussein \\ aus \\ Uelzen
}

Göttingen 2014 
Dekan: Prof. Dr. rer. nat. H. K. Kroemer

I. Berichterstatter: Prof. Dr. med. C. Herrmann-Lingen

II. Berichterstatter: Prof. Dr. med. C. Spitzer

III. Berichterstatter: Prof. Dr. med. dent. R. Mausberg

Tag der mündlichen Prüfung: 01.06.2015 


\section{INHALTSVERZEICHNIS}

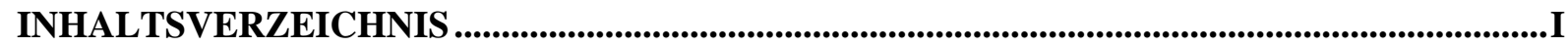

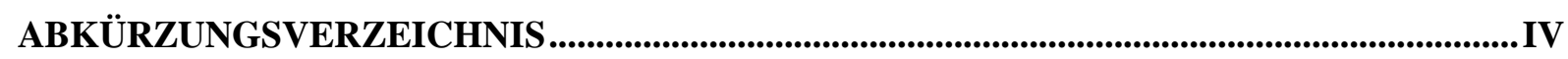

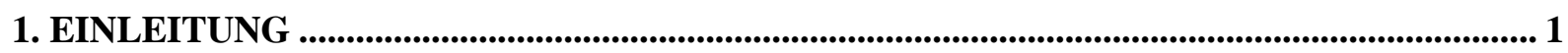

1.1 Perkutane Koronarintervention ............................................................................. 1

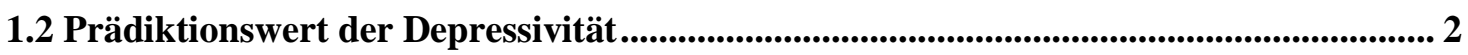

1.3 Historische Entwicklung und Prognose der Typ-D-Persönlichkeit................................. 4

1.4 Prognose der Angst in der aktuellen Literatur ................................................................ 7

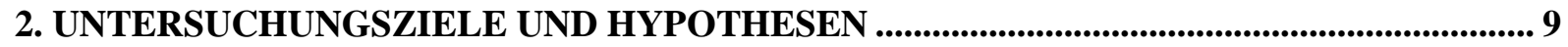

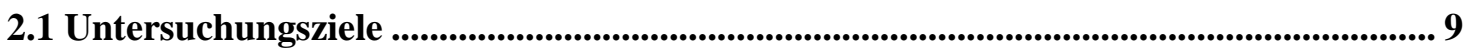

2.2 Hypothesen ......................................................................................................................................................... 9

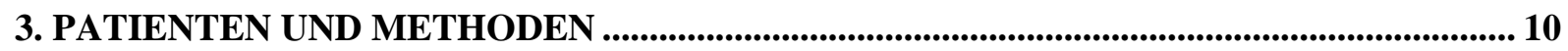

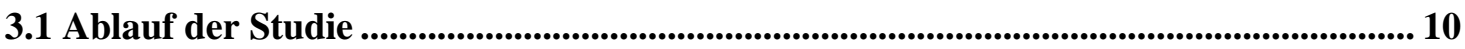

3.2 Ablauf der Nachverfolgung ......................................................................................................... 10

3.3 Ein- und Ausschlusskriterien .................................................................................................................. 11

3.4 Verarbeitung der Daten und Statistik ........................................................................ 11

3.5 Methoden zur Überprüfung der Hypothesen .................................................................... 11

3.5.1 Hospital Anxiety and Depression Scale.......................................................... 11

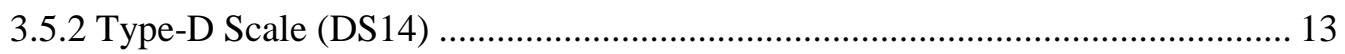

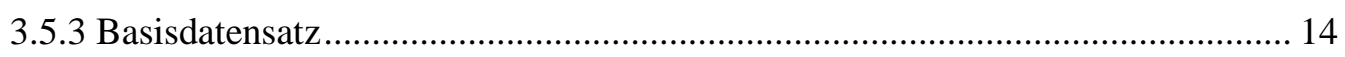

3.5.4 Major Adverse Cardiovascular Events und Revaskularisation........................... 15

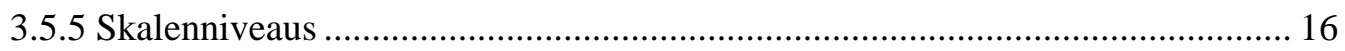

3.5.6 Statistische Methoden für den Vergleich von Gruppen..................................... 16

3.5.7 Verteilung der Angst- und Depressivitätswerte................................................. 16

3.5.8 Überprüfung der 1. und der 2. Hypothese: Einfluss der Depressivität und der

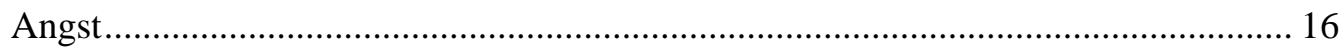

3.5.9 Überprüfung der 3. Hypothese: Einfluss der Typ-D-Persönlichkeit.................... 18

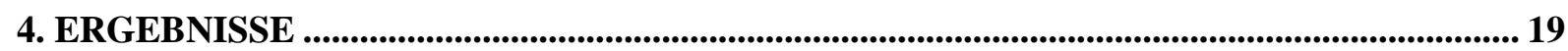

4.1 Beschreibung der Stichprobe ............................................................................................. 19

4.1.1 Soziodemographische Daten der Probanden .................................................... 19

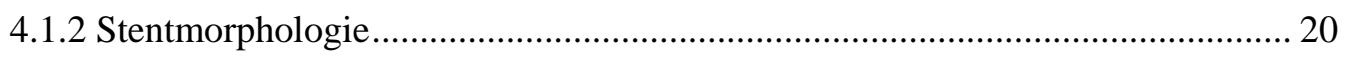

4.1.3 Kardiologische Risikofaktoren und Vorerkrankungen der Studienteilnehmer... 20

4.1.4 Beantwortung der Fragebögen bei Aufnahme .................................................. 20

4.1.5 Ergebnisse der HADS- und DS14-Fragebögen bei Einschluss ......................... 21 
4.1.6 Häufigkeiten der Endpunkte innerhalb des Nachbeobachtungszeitraums 21

4.2 Überprüfen der 1. Hypothese: Prognostischer Einfluss der Depressivität 22

4.2.1 Vergleich der Subgruppen mit und ohne Depressivität .................................... 23

4.2.2 Testung der 1. Hypothese: Depressivität hat keinen Effekt auf das Überleben.. 23

4.2.3 Einflussnahme somatischer Basisdaten auf den Zusammenhang der Gesamtmortalität mit der Depressivität 25

4.2.4 Kein Einfluss der Depressivität auf die Major Adverse Cardiovascular Events 26 4.2.5 Einflussnahme somatischer Basisdaten auf den Zusammenhang der MACEs mit

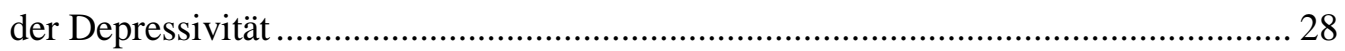

4.2.6 Kein Einfluss der Depressivität auf die Revaskularisationsrate ........................ 29

4.2.7 Einflussnahme somatischer Basisdaten auf den Zusammenhang der Revaskularisationsrate mit der Depressivität

4.2.8 Explorative Zusatzanalyse: Depressivität hat Einfluss auf die Gesamtmortalität im Nachbeobachtungszeitraum von 2 Jahren .... 32

4.2.9 Einflussnahme somatischer Basisdaten auf die 2-jährige Gesamtmortalität bei Patienten mit Depressivität 32

4.2.10 Verwerfen der 1. Hypothese ………............................................................ 33

4.3 Überprüfen der 2. Hypothese: Prognostischer Einfluss der Typ-D-Persönlichkeit.... 33

4.3.1 Gruppenvergleich der Patienten mit und ohne Typ-D-Persönlichkeit. .35

4.3.2 Testung der 2. Hypothese: Kein Einfluss von Typ-D auf die Gesamtmortalität 35 4.3.3 Einflussnahme somatischer Basisdaten auf den Zusammenhang der

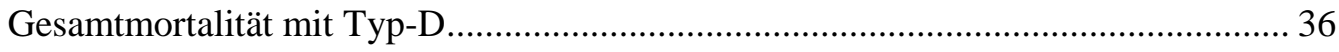

4.3.4 Kein Einfluss von Typ-D auf das kumulative ereignisfreie Überleben . 37

4.3.5 Einflussnahme somatischer Basisdaten auf das kumulative ereignisfreie Überleben bei Typ-D 39

4.3.6 Kein Einfluss von Typ-D auf die Revaskularisationsrate 39

4.3.7 Einflussnahme somatischer Basisdaten auf den Zusammenhang der Revaskularisations-rate mit Typ-D .

4.3.8 Negative Affektivität, soziale Inhibition und ihr Interaktionsterm als Prädiktoren

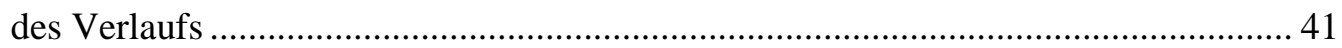

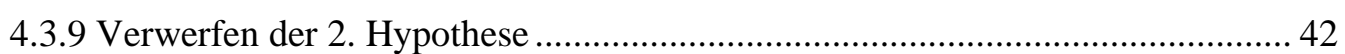

4.4 Überprüfen der 3. Hypothese: Prognostischer Einfluss der Angst.................................. 42

4.4.1 Gruppenvergleich von ängstlichen und nicht-ängstlichen Patienten .................. 43

4.4.2 Testung der 3. Hypothese: Angst ist mit verbessertem Überleben assoziiert..... 44

4.4.3 Einflussnahme somatischer Basisdaten auf den Zusammenhang der

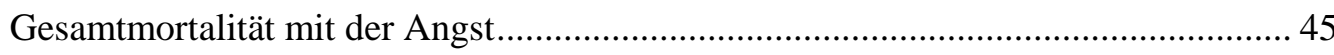

4.4.4 Protektiver Einfluss der Angst auf die Major Adverse Cardiovascular Events .. 46 4.4.5 Einflussnahme somatischer Basisdaten auf den Zusammenhang der MACEs mit der Angst 48

4.4.6 Erhöhte Revaskularisationsraten bei Angst im obersten Quartil . 49

4.4.7 Einflussnahme somatischer Basisdaten auf den Zusammenhang der

Revaskularisations-rate mit der Angst. 


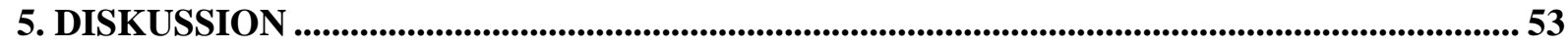

5.1 Studienziel und Resultat .......................................................................................53

5.2 Studiendesign und Begrenzungen der Studie ................................................................ 54

5.3 Repräsentativität der Kohorte ............................................................................... 55

5.3.1 Soziodemographische Angaben........................................................... 55

5.3.2 Nachbeobachtungszeitraum und Endpunkte ......................................... 56

5.3.3 Prävalenz der Typ-D-Persönlichkeit...................................................... 58

5.4 Diskussion der 1. Hypothese: Prognostische Effekte der Depressivität ........................ 59

5.5 Diskussion der 2. Hypothese: Prognostische Effekte der Typ-D-Persönlichkeit......... 61

5.6 Diskussion der 3. Hypothese: Prognostische Effekte der Angst ................................ 64

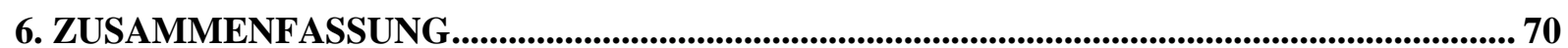

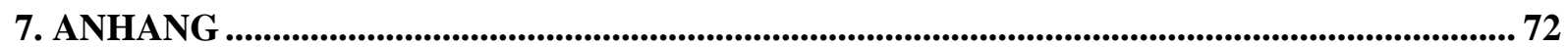

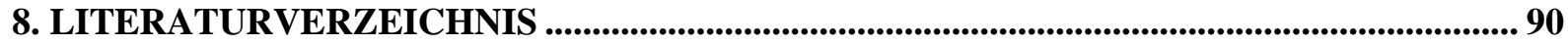

9. VERÖFFENTLICHUNGEN MIT BEZUG ZUR DISSERTATIONSSCHRIFT .................. 103 


\section{ABKÜRZUNGSVERZEICHNIS}

\begin{tabular}{ll}
\hline BMI & Body-Mass-Index \\
\hline BMS & Bare Metal Stent \\
\hline CI & Confidence Interval, Konfidenzintervall \\
\hline DES & Drug-Eluting Stent \\
\hline DS14 & Type-D Scale 14 \\
\hline HADS & Hospital Anxiety and Depression Scale \\
\hline HR & Hazard Ratio \\
\hline ICD & implantierbarer Kardioverter-Defibrillator \\
\hline KHK & koronare Herzkrankheit \\
\hline MACE & Major Adverse Cardiovascular Events \\
\hline MI & Myokardinfarkt \\
\hline NA & negative Affektivität \\
\hline NT-proANP & N-terminal pro-atrial natriuretic peptide \\
\hline PCI & perkutane Koronarintervention \\
\hline SD & Standard Deviation, Standardabweichung \\
\hline SI & soziale Inhibition \\
\hline
\end{tabular}




\section{EINLEITUNG}

\subsection{Perkutane Koronarintervention}

Die perkutane Koronarintervention, kurz PCI, ist ein modernes und hoch effektives, interventionelles Therapieverfahren, das 1977 das erste Mal zum Einsatz kam (Grüntzig et al. 1979). Mit fortwährender Verbesserung von Technik und Material hat es sich bis heute zu einer routinemäßigen Therapie zur Behandlung von koronaren Gefäßengen etabliert. Durch das Einbringen eines Stents auf Höhe der Stenose können zum einen pektanginöse Herzbeschwerden schnell und wirksam beseitigt werden, zum anderen verringert sich die Notwedigkeit einer erneuten Revaskularisationstherapie. Voraus geht die koronare Herzkrankheit (KHK), die durch arteriosklerotische Veränderungen der Tunica intima und der Tunica media verursacht wird. Nach wie vor gilt die KHK weltweit als häufigste Todesursache (MüllerNordhorn und Willich 2008; Franco et al. 2011). Verantwortlich dafür ist die Arteriosklerose der Koronararterien, die zu Gefäßengpässen führt. Diese entstehen durch Einlagerungen von Lipiden, Kalk und Bindegewebe, was zur Reduktion der Sauerstoffbereitstellung des Myokards führt. Daraus resultiert ein Ungleichgewicht von Sauerstoffbedarf und Angebot, welches sich im Fall einer stabilen Angina pectoris in Form von retrosternal lokalisierten Schmerzen zeigen kann. Bei einem akuten Verschluss im Sinne eines Myokardinfarktes kann es zur ischämischen Myokardnekrose kommen. Als kardiovaskuläre Risikofaktoren für die Ausbildung von Gefäßplaque gelten neben erhöhtem Lebensalter und dem männlichen Geschlecht (Müller-Nordhorn und Willich 2008) das Vorhandensein einer Hyperlipoproteinämie (Secretariat 2008), eines Diabetes mellitus (Huxley et al. 2006), einer arteriellen Hypertonie sowie Tabakabusus und eine positive Familienanamnese (Perk et al. 2012; Tunstall-Pedoe et al. 1999; Link und Tanner 2001; Ebrahim und Smith 1997). Außerdem sind psychosoziale Risikofaktoren wie niedriger Sozialstatus, fehlende soziale Unterstützung und beruflicher Stress bekannt (Perk et al. 2012). Eine Übersicht der Risikofaktoren zeigt Tabelle 1. 
Tabelle 1: Risikofaktoren der koronaren Herzkrankheit

- erhöhtes Lebensalter

- männliches Geschlecht

- Hyperlipoproteinämie

- Diabetes mellitus
- arterielle Hypertonie

- Tabakabusus

- positive Familienanamnese

- psychosozialer Stress

Die Angina pectoris-Symptomatik kann mittels Koronarangioplastie verbessert werden, da eine myokardiale Perfusionssteigerung und folglich eine Zunahme der körperlichen Belastbarkeit ermöglicht wird. Bedauerlicherweise führt das elektive Einbringen eines Koronarstents gegenüber optimaler konservativer Behandlung weder zu einem signifikanten Rückgang der Mortalitätsrate noch verringert es das Auftreten eines Myokardinfarktes. Dennoch lassen sich positive Effekte auf die Restenose und auf akute Verschlüsse betroffener Gefäße nach Ballondilatation nachweisen (Schömig et al. 2008). Bei einem Vergleich mit Patienten, die ausschließlich eine optimierte pharmakologische Therapie erhalten, verlängert auch das Implantieren eines Drug-Eluting Stents (DES) nicht signifikant das Überleben (Schömig et al. 2008; Trikalinos et al. 2009). Dies weist darauf hin, dass die invasive Intervention allein keine hinreichende Erklärung für die Prognose bringen kann und schafft Raum für Überlegungen, die zu Hypothesen führen, die besagen, dass vielmehr andere somatische oder psychosoziale Faktoren prognostisch bedeutsam sein könnten und nicht zwangsläufig direkte, hämodynamische Effekte am betroffenen Koronargefäß.

\subsection{Prädiktionswert der Depressivität}

Neben den bekannten Risikofaktoren wurde in der Vergangenheit für KHK-Patienten akuter und chronischer Stress mit einer negativen Prognose in Verbindung gebracht (Rozanski et al. 2005). Nachdem gezeigt wurde, dass die elektive PCI prognostisch wenig bedeutsam ist, psychosoziale Faktoren aber mutmaßlich in der Atherogenese eine wichtige Rolle spielen, wurden psychosoziale Faktoren auch als Prognosemarker untersucht. Es ist dargestellt worden, dass Depressivität bei bestehender koronarer Herzerkrankung eine häufige psychische Begleiterscheinung darstellt und mit einer verschlechterten Prognose einhergeht (Nicholson et al. 2006; Barth et al. 2004; Herrmann-Lingen und Buss 2002). Es gibt unterschiedliche Ursachen, die in Verbindung mit Depressivität ein negatives Outcome (engl.: klinisches Ergebnis) 
nach sich ziehen. Zum einen könnten depressive Patienten ein nachteiliges Gesundheitsverhalten aufweisen, das sich durch Mangel an Bewegung, Tabakabusus, Missachtung von Diätverordnungen und verminderte Adhärenz bei der Medikamenteneinnahme äußert (Ladwig et al. 2013). Zum anderen spielen neurophysiologische Prozesse wie die Aktivierung des sympathischen Nervensystems bei dem Vorhandensein einer Depressivität eine Rolle. Herzfrequenz und Blutdruck werden so in die Höhe getrieben. Auch die Wirkung des veränderten Kortisolspiegels nimmt Einfluss auf das weitere Krankheitsgeschehen (Vreeburg et al. 2009).

Bei einer relativ hohen Inzidenz von ca. 30\% stellten sich bereits zahlreiche Autoren die Frage nach dem prädiktiven Wert dieses psychischen Parameters und kamen dabei, v.a. bei Betrachtung unterschiedlicher Beobachtungszeiträume und bei Analyse verschiedener Patientengruppen, zu folgenden Ergebnissen:

Für ein kurzes Follow-Up-Intervall (1-3 Jahre) zeigte sich bei PCI-Patienten, dass der weitere, nachteilige Krankheitsverlauf positiv mit dem Vorhandensein depressiver Symptome assoziiert ist (Carney et al. 1988; Pedersen et al. 2007a; Denollet et al. 2007; Pedersen et al. 2009). Nicht-fataler Myokardinfarkt und Tod waren in diesen Untersuchungen Endpunkte und der Grad der Depressivität wurde mittels Fragebögen erfasst. Studien, die sich über die gleiche Nachbeobachtungsdauer allgemein mit KHK-Patienten beschäftigten, kamen zu ähnlichen Ergebnissen und stellten ebenfalls den prognostischen Wert der Depressivität für kardiale und nicht-kardiale Ereignisse fest (Rothenbacher et al. 2007; Frasure-Smith und Lesperance 2008; Nakamura et al. 2013). Eine KHK-Metaanalyse von 62 publizierten prospektiven Studien ermittelte eine Verdoppelung des Mortalitätsrisikos innerhalb von 2 Jahren Nachbeobachtung. Dieser Effekt zeigte sich unabhängig von anderen Risikofaktoren (Barth et al. 2004). Für einen Zeitraum von 18 Monaten nach Myokardinfarkt wurde ein erhöhtes Risiko für kardiale Mortalität festgestellt (Frasure-Smith et al. 1995a). Zu ähnlichen Ergebnissen kamen zahlreiche Studien, die depressive Postmyokardinfarktpatienten für eine Nachbeobachtungsdauer von durchschnittlich 13,7 Monaten analysierten (van Melle et al. 2004). Eine Metaanalyse über 25 Jahre mit einer durchschnittlichen Nachbeobachtungszeit von 16 Monaten zeigte ein 1,6-2,7-fach erhöhtes Mortalitätsrisiko innerhalb von 2 Jahren nach Myokardinfarkt (Meijer et al. 2011). Sowohl für Herzinsuffizienzpatienten (Rutledge et al. 2006) als auch für gemischte Patientengruppen (Herrmann et al. 1998) konnte ein unabhängiger Effekt der selbsteingeschätzten Depressivität auf das Überleben nachgewiesen werden. 
Demgegenüber stehen einige Studien, die keinen Effekt der Depressivität auf die Prognose von Herzpatienten in einem Nachbeobachtungszeitraum von 6-18 Monaten feststellen konnten (Mayou et al. 2000; Lane et al. 2001; Schmidt et al. 2011).

Bei der Analyse des Prädiktionswertes der Depressivität in Langzeitanalysen (ab 4 Jahren) ergab sich ein ausgeglicheneres Forschungsbild. In einem 7-jährigen Nachbeobachtungszeitraum zeigte sich ein 1,6-fach erhöhtes Mortalitätsrisiko bei Patienten nach Koronarstentintervention (Damen et al. 2013). In einer Studie mit 1250 KHK-Patienten und einem Follow-Up von bis zu 19,4 Jahren kamen die Autoren zu einem ähnlichen Ergebnis (Barefoot et al. 2000). Die Prognose von Postmyokardinfarktpatienten war in einem Nachbeobachtungszeitraum von 5 Jahren (Lesperance et al. 2002; Grace et al. 2005) und von 10 Jahren (Welin et al. 2000) negativ von der Depressivität beeinflusst worden. Herzinsuffizienzpatienten wurden in einer Metaanalyse in Bezug auf die Prognose untersucht, wobei sich Depressivität als konsistenter Prädiktor für ein nachteiliges Outcome herausstellte (Rutledge et al. 2006).

In anderen Studien war der Zusammenhang zwischen der Depressivität und der Mortalität in Langzeitbeobachtungen nicht signifikant. Dies zeigte sich bei PCI-Patienten (Sullivan et al. 2003), bei Postmyokardinfarktpatienten (Stewart et al. 2003; Parakh et al. 2008) und in Patientenkohorten mit unterschiedlichen kardiovaskulären Erkrankungen (Herrmann et al. 2000; Kornerup et al. 2011).

Die heutige Studienlage spricht trotz widersprüchlicher Ergebnisse eher für einen prognostisch bedeutsamen Einfluss der Depressivität bei KHK. Dieser klinisch bedeutsame Zusammenhang sollte daher in der vorliegenden Arbeit ein maßgeblicher zu untersuchender Faktor werden.

\subsection{Historische Entwicklung und Prognose der Typ-D-Persönlichkeit}

Ein weiterer psychischer Parameter, der in dieser Studie auf die Prognose der Patienten sowie auf das weitere Krankheitsgeschehen untersucht werden sollte, ist die Typ-D-Persönlichkeit. Historischer Vorläufer dieser distressed personality war das Typ-A-Verhaltensmuster, das in den achtziger Jahren Grundlage zahlreicher psychokardiologischer Studien war. Eine Person mit Typ-A-Verhalten sieht sich selbst als Konkurrent zu seinen Mitmenschen und zeigt sich in seinen Handlungsweisen aggressiv und kompetitiv. Das Gefühl, ständig unter Zeitdruck zu stehen, veranlasst ihn, immer mehr in immer weniger Zeit ermöglichen zu wollen (Rosenman et al. 1975). Ein erhöhtes Risiko, beim Vorhandensein des Typ-A-Verhaltensmusters eine koronare Herzkrankheit auszubilden, konnte zum Beispiel in der Western Collaborative 
Group Study an 3154 gesunden männlichen Probanden nachgewiesen werden (Rosenman et al. 1975).

Folgestudien konnten dieses Phänomen jedoch nicht einheitlich replizieren, sodass die Typ-AHypothese zunehmend angezweifelt wurde. Die Forschung in den neunziger Jahren brachte einen neuen Persönlichkeitstypus hervor, der möglicherweise besser mit der koronaren Herzkrankheit korreliert (Denollet 1993). Denollet stellte erstmals im Jahre 1995 diesen psychischen Parameter vor, der das emotionale Erleben des Menschen widerspiegelt und grundsätzlich in zwei verschiedene Dimensionen eingeteilt werden kann (Denollet 1995). Zum einen wird die negative Affektivität (NA) gemessen, die die Neigung des Menschen beinhaltet, sich besorgt, unglücklich und ärgerlich zu fühlen und die als stabiles Persönlichkeitsmerkmal betrachtet wird. Vergleichbar ist die negative Affektivität mit dem Neurotizismus. Auf der anderen Seite steht die soziale Inhibition (SI). In Anwesenheit unbekannter Mitmenschen fühlen sich diese Personen unsicher und gehemmt (Denollet 2005). Negative Gefühle werden bei hoher Ausprägung dieses Merkmals bewusst unterdrückt und führen in kritischen Situationen zu einem erhöhten Stressempfinden (Denollet 1993; Denollet 1998). Dieser Stress entsteht vor allem, wenn eine Person sowohl unter negativer Affektivität als auch unter sozialer Inhibition leidet. Daraus resultiert die Annahme, dass erst das Zusammenspiel dieser beiden Merkmale nachteilige Effekte auf den Verlauf kardiovaskulärer Erkrankungen hat. Dieses wurde von Denollet als Typ-D-Persönlichkeit bezeichnet (Denollet 2000; Denollet et al. 2010). Diese ungünstigen Effekte zeigten sich bei Patienten mit ischämischer Herzerkrankung und eingeschränkter linksventrikulären Ejektionsfraktion (Denollet 1998). Einige Emotionen, die die negative Affektivität und die soziale Inhibition charkterisieren, zeigt Tabelle 2.

Tabelle 2: Das Zusammenspiel aus NA und SI führt zur Typ-D-Persönlichkeit

\begin{tabular}{|l|l|}
\hline $\begin{array}{l}\text { Negative Affektivität (NA) beinhaltet: } \\
\text { - sich sorgen }\end{array}$ & $\begin{array}{l}\text { Soziale Inhibition (SI) beinhaltet: } \\
\text { - Unsicherheit } \\
\text { - oft unglücklich sein } \\
\text { - sich häufig ärgern }\end{array}$ \\
\hline
\end{tabular}

Beim Vergleich der Häufigkeiten des Typ-D-Musters in verschiedenen Studien variierte der Anteil von 38,5\% (Williams et al. 2008) bis 18\% (Bergvik et al. 2010), wobei Werte zwischen $20 \%$ und $30 \%$ häufig waren. 
Vorangegangene Studien zeigten, dass Charakterzüge wie Feindseligkeit und Neigung zum Ärger Hauptfaktoren für chronischen Stress sind. Dieser kann pathologische kardiale Ereignisse bei vorerkrankten Patienten zur Folge haben (Chida und Steptoe 2009). Eine Serie an Studien der Arbeitsgruppe um Denollet aus Antwerpen bzw. Tilburg zeigte Zusammenhänge zwischen der Typ-D-Persönlichkeit und erhöhter Mortalität. Auch ein vermehrtes Aufkommen klinischer Ereignisse im weiteren Verlauf konnte nach Adjustierung von somatischen Erkrankungen und Symptomen von Depressionen nachgewiesen werden (Denollet et al. 1996; Denollet und Brutsaert 1998; Denollet et al. 2000; Pedersen et al. 2004; Denollet et al. 2006a; Pedersen et al. 2007b; Martens et al. 2010). Die genannten Autoren beschrieben die Typ-DPersönlichkeit als einen unabhängigen Prädiktor für klinische Ereignisse wie kardialen Tod, Myokardinfarkt und invasive Maßnahmen im Sinne einer Revaskularisation. Weitere klinische Studien zeigten für Patienten mit Typ-D-Persönlichkeit nachteilige Effekte auf den subjektiven Gesundheitsstatus nach Myokardinfarkt (Denollet et al. 1995; Mols et al. 2010). Studienteilnehmer mit Herzinsuffizienz (Schiffer et al. 2008) und koronarer Bypass-Operation (Al-Ruzzeh et al. 2005) hatten ebenfalls eine verschlechterte Prognose im weiteren Krankheitsverlauf. Des Weiteren wurde nachgewiesen, dass Patienten mit Typ-D-Muster, nach Einsetzen eines implantierbaren Kardioverter-Defibrillators (ICD), vermehrt unter lebensbedrohlichen Arrhythmien litten (van den Broek et al. 2009). Für ICD-Patienten wurde darüber hinaus eine erhöhte Mortalitätsrate, unabhängig von der Schockabgabefrequenz, nachgewiesen (Perdersen et al. 2010). In der Allgemeinbevölkerung zeigte sich vermehrter Disstress bei Menschen mit Typ-D-Persönlichkeit (Beutel et al. 2012). Eine Ursache für diese potenziell gesundheitsschädlichen Effekte der Typ-D-Persönlichkeit wurde in einer erhöhten Kortisolkonzentration gesehen bzw. durch erhöhte Kortisolspiegel am Morgen (cortisol awakening response) erklärt (Whitehead et al. 2007; Molloy et al. 2008). Weitere mögliche Faktoren sind nach Studienlage exzessive sympathische Aktivität (Habra et al. 2003), erhöhte oxidative Stressbelastung (Kupper et al. 2009) und eine dysfunktionale pro- zu antiinflammatorische Zytokinbalance (Conraads et al. 2006; Denollet et al. 2009a).

Auf der anderen Seite stehen Studien, die den beschriebenen positiven Zusammenhang bei kardiovaskulären Patienten mit unterschiedlichen Krankheitsentitäten nicht darstellen konnten. Das Typ-D-Muster konnte in diesen Studien (Pelle et al. 2010; Coyne et al. 2011; Grande et al. 2011; Volz et al. 2011; Damen et al. 2013) nicht als Ursache eines nachteiligen kardiovaskulären Krankheitsverlaufs ermittelt werden, was methodische Zweifel über den tatsächlichen prognostischen Wert dieses psychologischen Merkmals auslöst. Es ist demnach frag- 
lich, ob die Typ-D-Persönlichkeit einen prognostischen Indikator für Mortalität bei kardiovaskulären Patienten darstellt.

Angesichts dieser kontroversen Resultate bei kardiovaskulären Patienten stellt sich die Frage der prognostischen Relevanz des Typ-D-Konstrukts, weswegen weitere klinische Studien in größeren Kohorten notwendig sind (Smith 2011; Coyne und de Voogd 2012). In der vorliegenden Studie sollte aus diesem Grund der unabhängige Einfluss der Typ-D-Persönlichkeit (sowie der beiden zugrunde liegenden Subskalen) bei Patienten nach PCI untersucht werden.

\subsection{Prognose der Angst in der aktuellen Literatur}

Angst ist eine Grundempfindung, die Gefühle von Beklemmung, Unsicherheit und Ausgeliefertsein beinhaltet und in unterschiedlichen Zusammenhängen auftreten kann. Sie entsteht bei Menschen als Imaginär- oder Realangst und hat evolutionsgeschichtlich eine sehr wichtige Funktion. In vermeintlichen Gefahrensituationen leitet die Angst angemessene Verhaltensmuster wie Flucht ein und aktiviert körpereigene Schutzmechanismen. Ängstliche Patienten leiden häufig unter einer unguten Vorahnung und Furcht, wobei unterschiedliche Auslöser bekannt sind, darunter offene Plätze und soziale Situationen (Emilien et al. 2002).

Bisher wurde in der Literatur die Angst als mutmaßlicher prognostischer Faktor für die Progression einer vorbestehenden koronaren Arteriosklerose weitestgehend außer Acht gelassen. Aktuelle Daten geben keine sichere Erkenntnis über die Angst als prognostischen Faktor für Mortalität oder Myokardinfarkt, da sich wissenschaftliche Studien in diesem Punkt widersprechen. Bisher wurde gezeigt, dass Angst, die oft bei Herzpatienten vorliegt und häufig mit Depressivität einhergeht (Denollet et al. 2006b; Pajak et al. 2013), die Rate eines tödlichen ischämischen Infarkts erhöht. Ebenso steigt das Risiko für Myokardinfarkt und kardiovaskulären Tod bei ängstlichen Patienten (Roest et al. 2010a). Des Weiteren wurde nachgewiesen, dass Angst einen nachteiligen Effekt auf die Ausprägung depressiver Symptome und auf den Gesundheitszustand nach PCI haben kann (Pedersen et al. 2006), jedoch zu einer erhöhten Gesundheitsfürsorge (gemessen an der Frequenz des Arztkontakts) nach akutem Myokardinfarkt führt (Strik et al. 2003). Im Gegensatz zur Depression, für die in zahlreichen Studien eine erhöhte Mortalität und Morbidität nachgewiesen wurde, ist für den prognostischen Einfluss der Angst bei KHK-Patienten wenig bekannt (Sullivan et al. 2000; Bui et al. 2010; Roest et al. 2010a; Schmidt et al. 2011).

Einige Studien zeigten sogar, dass das Vorhandensein von Angst bei Patienten mit bestehender KHK und ohne Myokardinfarkt in der Vorgeschichte ein verbessertes Überleben nach 
sich zieht (Meyer et al. 2010). Für Menschen aus der Allgemeinbevölkerung stellte man ebenfalls einen positiven Zusammenhang zwischen Angst und Überleben fest (Mykletun et al. 2007). Folglich wurde in dieser prospektiven Studie der Einfluss der Angst auf die Prognose der Patienten nach PCI untersucht. 


\section{UNTERSUCHUNGSZIELE UND HYPOTHESEN}

Ziel der vorliegenden Studie war es, den Einfluss von psychischen Faktoren auf kardiale und nicht-kardiale vaskuläre Ereignisse (zusammengefasst als Major Adverse Cardiovascular Events bzw. Revaskularisation) sowie die Gesamtsterblichkeit nach PCI zu untersuchen. Die psychischen Parameter als Ausgangswerte für das weitere beobachtete Krankheitsgeschehen nach PCI waren dabei das Vorhandensein von Angst, von Depressivität sowie das Vorliegen einer Typ-D-Persönlichkeit. Bei einem Vergleich der internationalen Studienergebnisse bei KHK-Patienten zeigte sich in Bezug auf die prognostische Vorhersagekraft der vorgestellten psychischen Parameter kein einheitliches Bild.

\subsection{Untersuchungsziele}

1.) Es soll der unabhängige prädiktive Wert der Depressivität 5 Jahre nach PCI mittels Betrachtung schwerer kardiovaskulärer Ereignisse sowie der Gesamtmortalität analysiert werden.

2.) Der unabhängige prognostische Wert der Typ-D-Persönlichkeit für das Auftreten eines schweren kardiovaskulären Ereignisses und für die Mortalitätsrate innerhalb von 5 Jahren nach PCI soll bestimmt werden.

3.) Es soll der unabhängige prädiktive Wert der Angst 5 Jahre nach PCI mittels Betrachtung schwerer kardiovaskulärer Ereignisse sowie der Gesamtmortalität analysiert werden.

\subsection{Hypothesen}

1.) Für Patienten mit Depressivität besteht ein erhöhtes Risiko für das Auftreten eines schweren kardiovaskulären Ereignisses und eine erhöhte Gesamtmortalität innerhalb von 5 Jahren nach PCI.

2.) Das Vorliegen einer Typ-D-Persönlichkeit hat einen Effekt auf das Auftreten eines schweren kardiovaskulären Ereignisses und auf die Gesamtmortaliät innerhalb der 5 Jahre nach PCI.

3.) Der Parameter Angst hat Einfluss auf das Auftreten eines schweren kardiovaskulären Ereignisses und auf die Gesamtmortalität innerhalb von 5 Jahren nach PCI. 


\section{PATIENTEN UND METHODEN}

\subsection{Ablauf der Studie}

Im Zeitraum von November 2004 bis Juni 2006 füllten 470 Patienten im Rahmen der Routine vor einer elektiven Koronarstentintervention im Herzkatheterlabor des Bremer Lehrklinikums Links der Weser die Fragebögen aus. Bezogen auf die patientenorientierten, psychologischen Auskünfte wurde über die ärztliche Schweigepflicht aufgeklärt. Nach erfolgter Stentintervention wurden die ausschließlich volljährigen Patienten mit Thrombozytenaggregationshemmern behandelt. Die Ergebnisse der zwei unterschiedlichen psychometrischen Fragebögen wurden 5 Jahre später retrospektiv für die Verlaufsvorhersage verwendet. Im späteren Verlauf erfolgte für die statistische Auswertung der Daten eine Pseudonymisierung.

Ein positives Votum über dieses Studiendesign liegt sowohl seitens der Bremer als auch seitens der Göttinger Ethikkommission vor.

\subsection{Ablauf der Nachverfolgung}

Um der Fragestellung nachzugehen, ob bei den Patienten innerhalb der 5 Jahre nach Koronarstentintervention ein kardiales oder nicht-kardiales Ereignis aufgetreten ist, wurde in den vor Ort archivierten Patientenakten im Herzkatheterlabor recherchiert. Viele Patienten kamen nach dem erfolgten Eingriff regelmäßig in die kardiologische Praxis des Klinikums Links der Weser, um sich einem festgelegten Nachsorgeprogramm zu unterziehen. Kam es erneut zur Implantation eines Koronarstents, konnte dieses mittels Datum und Herzkatheterprotokoll nachvollzogen werden. Auch weitere kardiale oder nicht-kardiale Ereignisse wurden auf diese Weise dokumentiert und konnten für die Datenerfassung genutzt werden.

War eine Nachverfolgung auf diesem Weg nicht möglich, wurden die Patienten 5 Jahre nach initialer Koronarstentimplantation telefonisch kontaktiert und zum Krankheitsverlauf befragt. Bei den Studienteilnehmern, die telefonisch nicht erreichbar waren, konnte der behandelnde Hausarzt telefonisch Auskunft geben. Weiterhin bekamen nicht kontaktierbare Patienten einen Brief mit der Bitte, über eventuell in der Zwischenzeit aufgetretene kardiale oder nichtkardiale Ereignisse zu berichten. Soziodemographische Angaben zu den Patienten wurden aus einem Basisdatensatz entnommen. Diese anamnestischen Angaben wurden zum Zeitpunkt der Stentimplantation erhoben. 


\subsection{Ein- und Ausschlusskriterien}

Eingeschlossen wurden Patienten, die im Ausgangszeitraum einen De-novo-Koronarstent implantiert bekamen und in diesem Kontext routinemäßig die hier relevanten Fragebögen ausgefüllt hatten. Keine Rolle spielte dabei die Stentmorphologie im Sinne der Größe, des Durchmessers und der Beschichtung. Diese Angaben wurden gesondert dokumentiert.

Ausgeschlossen wurden Patienten, bei denen initial lediglich eine Ballondilatation ohne Stentimplantation durchgeführt wurde oder im Zuge der Herzkatheteruntersuchung gar keine Intervention notwendig war. Auch eine erneute Dilatation eines zuvor eingesetzten Stents war ein Ausschlusskriterium. Patienten mit akutem Koronarsyndrom nahmen ebenfalls nicht an der Studie teil. Des Weiteren führten Weigerung der Patienten, nicht ausreichende Deutschkenntnisse, floride Psychosen und schwere hirnorganische Beeinträchtigungen dazu, dass kein Fragebogen ausgefüllt worden war und ein Einschluss in die Studie nicht möglich war.

\subsection{Verarbeitung der Daten und Statistik}

Die patientenorientierten Daten wurden für die Studie pseudonymisiert in eine Microsoft Excel-Datei eingegeben und gesichert. Die sich anschließende Auswertung und das Erstellen von Graphen erfolgte mit Hilfe des Statistikprogramms SPSS Version 20 und Coral Draw (2003) für Windows. Für die Textverarbeitung kam die Software Microsoft Word (2011) zum Einsatz. Als Rate der fehlenden Werte (missing values) ergaben sich Werte zwischen 0\% (Alter und Geschlecht) und 2\% (Nachbeobachtungsrate).

Die psychometrischen Verfahren, die verwendeten Skalenniveaus und die Auswahl des entsprechenden statistischen Vorgehens zum Überprüfen der Hypothesen soll im Folgenden dargestellt werden.

\subsection{Methoden zur Überprüfung der Hypothesen}

\subsubsection{Hospital Anxiety and Depression Scale}

Um zu überprüfen, ob es einen Zusammenhang zwischen den psychischen Parametern Angst bzw. Depressivität und einem erneuten pathologischen Vorfall in den folgenden 5 Jahren nach Stentimplantation gibt, wurde die Hospital Anxiety and Depression Scale (HADS) unter dieser Fragestellung analysiert. Dieses international gut erprobte, psychometrische Instrument entwickelten Zigmond und Snaith im Jahr 1983 (Zigmond und Snaith 1983), woraufhin es 
sich im Laufe der Zeit als gängiges Instrument zur psychischen Beurteilung von Herzpatienten etablierte (Herrmann 1997; Spinhoven et al. 1997; Bjelland et al. 2002; Rothenbacher et al. 2007). Es erfasst als Selbstbeurteilungsverfahren die Ausprägung einer depressiven und ängstlichen Symptomatik innerhalb der vergangenen Woche (Herrmann et al. 1995). Der Fragebogen besteht aus 14 Items, die sich zu gleichen Teilen auf Angst und Depressivität beziehen, wobei jedes Item 4 Antwortmöglichkeiten bietet. Im Ankreuzverfahren entscheiden sich die Patienten, je nach Grad der Übereinstimmung, bei personenbezogenen Fragen wie „Ich fühle mich angespannt und überreizt“ für Antwortmöglichkeiten von z.B. „meistens“ bis „überhaupt nicht“. Bei jedem Item können bei 4 Antwortmöglichkeiten jeweils Werte von 0 bis 3 erreicht werden. Bei 7 Fragen pro Subskala ergibt sich insgesamt eine Spannbreite des Gesamtscores von 0 bis 21, wobei höhere Werte einen vermehrten Grad von Angst bzw. von Depressivität anzeigen. So kann der Grad psychischer Beeinträchtigungen von vorwiegend somatisch erkrankten Jugendlichen und Erwachsenen bestimmt werden.

Validität, Reliabilität und klinische Relevanz wurden in verschiedenen klinischen Settings erprobt, sodass sich die HADS in zahlreichen europäischen Ländern sowohl als Screeningverfahren als auch als Verlaufsparameter der Angst und der Depressivität durchsetzte (Herrmann et al. 1995). Mit einem Cronbach- $\alpha$-Wert von 0,80 für die Angst- und 0,82 für die Depressionsskala stellte die HADS ihre gute interne Konsistenz unter Beweis (Herrmann 1997).

Die HADS erfasst auch leichtere Ausprägungen und Störungsformen von Angst und Depressivität, die sich auf die Lebensqualität und die Prognose auswirken. Vorrangige Aspekte sind dabei Anhedonie und Interessenverlust. Beim Erheben der Angst geht es vorwiegend um das Screening allgemeiner Angstsymptome (Herrmann et al. 1995). Eine Störungsdiagnose ist wie bei Fragebögen generell aus den HADS-Werten nicht möglich. Wegen zu erwartender schiefer Verteilungen der Fragebogenergebnisse, die sich bei der HADS regelhaft finden, soll hier mit Quartilen gearbeitet werden. Je nach Grad der Angst bzw. der Depressivität werden die Patienten jeweils in vier Subgruppen eingeteilt und in Bezug auf den weiteren klinischen Verlauf analysiert. In der Literatur werden zumeist die Studienkohorten in ängstliche und nichtängstliche bzw. depressive und nicht-depressive Subgruppen unterteilt. Daher sollen auch hier die jeweils obersten Quartile, die für die stärksten Merkmalsausprägungen stehen im Vordergrund der Betrachtung stehen und mit den unteren drei Quartilen verglichen bzw. auf Effekte analysiert werden. Beispiele für Items aus der umfangreich validierten deutschen Version des HADS-Fragebogens zeigt Tabelle 3. 
Tabelle 3: Auszüge aus dem HADS-Fragebogen zur Bestimmung der Angst und der Depressivität. Der Grad der Übereinstimmung ist anzukreuzen.

- „ „Ich fühle mich angespannt und überreizt"“

- „Ich kann mich heute noch so freuen wie früher“ (negativ gepolt)

- „Mich überkommt eine ängstliche Vorahnung, dass etwas Schreckliches passieren könnte“

- „Ich kann lachen und die lustigen Dinge sehen“ (negativ gepolt)

- „Mir gehen beunruhigende Gedanken durch den Kopf“

\subsubsection{Type-D Scale (DS14)}

Zusätzlich zum HADS-Fragebogen bekamen die Patienten zum Zeitpunkt der PCI mit der Type-D Scale (DS14) ein weiteres psychologisches Messinstrument ausgehändigt. Dieser Fragebogen enthält die zwei Subskalen soziale Inhibition (SI) und negative Affektivität (NA) und misst das Vorhandensein einer Typ-D-Persönlichkeit. Entwickelt wurde er von dem Medizinpsychologen Denollet (Denollet 2005) und soll, anders als der HADS-Fragebogen, stabile Persönlichkeitseigenschaften beschreiben. In der Vergangenheit erwies sich die DS14 als reliable Messmethode mit einer hohen internen Konsistenz (Cronbach- $\alpha$-Wert>0,86) und adäquater Konstruktvalidität (Grande et al. 2004; Emons et al. 2007).

Jede der beiden Subskalen enthält 7 Items, die getrennt voneinander zum einen die soziale Inhibition und zum anderen die negative Affektivität messen. Jedes Item setzt sich aus einer fünfstufigen Likert-Skala zusammen, wobei sich der Patient zwischen Werten von 0 (,,trifft überhaupt nicht zu“) bis 4 (,trifft voll und ganz zu“) entscheiden muss. Auf diese Weise kann der Studienteilnehmer pro Subskala Werte zwischen 0 und 28 erreichen.

Mit der Subskala negative Affektivität werden Persönlichkeitsmerkmale wie Neigung zu Ärger, Gereiztheit, Besorgtheit und Pessimismus gemessen. Die Subskala soziale Inhibition beurteilt Tendenzen des Patienten, sich v.a. in Gegenwart fremder Menschen verschlossen, gehemmt und unsicher zu fühlen. Ein Auszug aus dem DS14-Fragebogen zur Bestimmung der Typ-D-Persönlichkeit zeigt Tabelle 4. 
Tabelle 4: Auszüge aus dem DS14-Fragebogen zur Bestimmung der Typ-D-Persönlichkeit. Der Grad der Übereinstimmung ist anzukreuzen.

- „Es fällt mir leicht, Kontakt zu anderen Menschen zu knüpfen“ (negativ gepolt)

- „Ich rege mich oft über unwichtige Dinge auf“

- „Ich fühle mich oft im Umgang mit Anderen gehemmt“

- „Ich fühle mich oft unglücklich“

- „Ich weiß nicht, worüber ich mit Anderen reden soll“

Kriterium für das Vorhandensein einer Typ-D-Persönlichkeit ist das Erreichen des festgelegten Cut-Off-Wertes von $\geq 10$ in beiden Subskalen (Denollet 2005). Dieser Cut-Off ist mit dem Median-Split gleichzusetzen, der in Validierungsstudien ermittelt wurde.

\subsubsection{Basisdatensatz}

Ein Basisdatensatz mit patientenbezogenen, soziodemographischen und anamnestischen Angaben wurde zu Beginn der Studie aus der Routinedokumentation übernommen. Dieser bildet die Grundlage um zu überprüfen, ob somatische bzw. demografische Variablen einen Effekt auf die Prognose der Patienten hatten. Der Basisdatensatz enthält sowohl somatische Angaben wie Alter, Gewicht und Vorerkrankungen als auch detaillierte Angaben zum implantierten Stent (vgl. Tabelle 5).

Tabelle 5: Der Basisdatensatz wurde bei Studienbeginn erstellt.

- Geschlecht

- Alter

- Größe

- Gewicht

- Body-Mass-Index

- Z.n. Bypass-Operation

- Z.n. Myokardinfarkt

- Diabetes mellitus bei Einschluss
- positive Familienanamnese

- Tabakabusus bei Einschluss

- Hypercholesterinämie bei Einschluss

- Hypertonie bei Einschluss

- Lokalisation des Stents (proximal vs. nicht proximal)

- Stentlänge, Stentdurchmesser

- $\quad$ Stent-Typ (DES oder BMS) 


\subsubsection{Major Adverse Cardiovascular Events und Revaskularisation}

Von entscheidender Bedeutung für diese Follow-Up-Studie war das Auftreten von gravierenden klinischen Ereignissen, zusammengefasst als Major Adverse Cardiovascular Events (MACE) und medizinisch indizierten Revaskularisationsmaßnahmen innerhalb der 5 Jahre nach PCI. Trat eines (oder mehrere) der in Tabelle 6 aufgelisteten Ereignisse im Beobachtungszeitraum auf, wurde dieses mit Datum dokumentiert. Unter Major Adverse Cardiovascular Events werden die Endpunkte akuter, nicht-fataler Myokardinfarkt, Apoplex und Tod verstanden. Da die genaue Todesursache nicht bei allen Patienten festzustellen war, werden die Endpunkte kardialer und nicht-kardialer Tod gemeinsam unter dem Aspekt der Gesamtmortalität betrachtet. Der Endpunkt Revaskularisation fasst die Ereignisse Re-PCI mit Stentimplantation, Re-PCI ohne Stentimplantation und Bypass-Operation zusammen (vgl. Tabelle $6)$.

Tabelle 6: Zusammengefasste Endpunkte innerhalb des Beobachtungszeitraums

\begin{tabular}{|l|l|}
\hline Revaskularisation & Major Adverse Cardiovascular Events \\
\hline - $\begin{array}{l}\text { erneute PCI mit Stentimplantati- } \\
\text { on }\end{array}$ & - Tod (kardial und nicht-kardial) \\
- PCI & - Apoplex \\
- Koronararterien-Bypass- \\
\begin{tabular}{l} 
Operation \\
\hline
\end{tabular}
\end{tabular}

Informationen $\mathrm{zu}$ den Endpunkten konnten zu großen Teilen aus der Patientenkartei des Herzkatheterlabors im Klinikum Links der Weser entnommen werden. Patienten, die einen Koronarstent implantiert bekamen, waren anschließend Teil eines regelmäßigen Nachsorgeprogramms und wurden vom behandelnden Kardiologen routinemäßig zum Krankheitsverlauf befragt und gegebenenfalls weiter untersucht bzw. behandelt. Aus diesen anamnestischen Angaben, die detailliert mit Datum versehen und chronologisch dokumentiert sind, konnten zahlreiche Endpunkte ermittelt werden.

Von besonderem Interesse waren dabei stattgefundene Revaskularisationsmaßnahmen und Major Adverse Cardiovascular Events innerhalb der 5 Jahre Beobachtungszeitraum nach Stentimplantation (vgl. Tabelle 6). Führte auch dieser Weg nicht zum gewünschten Erfolg, standen die jeweils behandelnden Hausärzte für studienrelevante Informationen zur Verfügung. 


\subsubsection{Skalenniveaus}

Für das Erfassen der für diese Studie relevanten Merkmale wurden unterschiedliche Skalenniveaus verwendet. Dabei richtete sich das Niveau nach der Art der Variablen, wodurch sich die Messqualitäten Nominalskala (Geschlecht, Diabetes mellitus vorhanden, Revaskularisation innerhalb der 5 Jahre Nachbeobachtung), Ordinalskala (Ergebnis der Fragebögen), Intervallskala (Zeit bis klinisches Ereignis) und Verhältnisskala (Alter, Größe, Gewicht) ergaben.

\subsubsection{Statistische Methoden für den Vergleich von Gruppen}

Demographische und klinische Daten sind als Mittelwerte \pm Standardabweichung (engl.: standard deviation, kurz SD), als Häufigkeiten und als Prozentangaben dargestellt. Mittelwerte von kontinuierlichen Variablen auf Intervallskalenniveau wurden mit Hilfe des Student-tTests verglichen. Dieses parametrische Testverfahren ermöglicht den Vergleich von Stichproben z.B. in den Variablen Alter, Body-Mass-Index und Stentlänge. Um kategoriale Variablen (z.B. Geschlecht, Tabakabusus) auf Nominalskalenniveau zu analysieren, kam der $\chi^{2}$-Test nach Pearson zum Einsatz. Für die Ereigniszeitberechnungen eignete sich die Kaplan-MeierAnalyse (inklusive log-rank Test) und für multivariable Analysen die Cox-Regression.

\subsubsection{Verteilung der Angst- und Depressivitätswerte}

Es sollte eine Grundlage geschaffen werden, um die untersuchte Studienkohorte im Sinne einer Ausprägung psychischer Parameter zu klassifizieren. Daher wurde mittels KolmogorowSmirnow-Test überprüft, ob die HADS-Werte einer Normalverteilung unterliegen. Lag keine Normalverteilung vor, erfolgte eine Einteilung der Patientenkohorte in Quartile. Für die Vergleichbarkeit der auf diese Weise entstandenen Subgruppen innerhalb der Kohorte konnten die Patienten je nach Fragebogen-Score in Fraktionen transformiert und auf signifikante Differenzen, bezogen auf die in 3.5.4 beschriebenen Endpunkte, untersucht werden. So wurden Merkmalausprägungen in Klassen unterteilt und miteinander verglichen.

\subsection{8 Überprüfung der 1. und der 2. Hypothese: Einfluss der Depressivität und der Angst}

Um die Hypothesen zu testen, eignete sich für den Häufigkeitenvergleich über alle Quartile sowie für den Vergleich der Patientengruppen mit und ohne auffälligem Fragebogenergebnis (4. gegenüber zusammengefasstes 1.-3. Quartil) der Chi-Quadrat-Test. Um zu überprüfen, ob es einen signifikanten Zusammenhang zwischen dem Vorhandensein der Angst bzw. der Depressivität und dem erneuten Auftreten eines klinischen kardiovaskulären Ereignisses (vgl. 
Tabelle 6) innerhalb der 5 Jahre nach PCI gibt, kam die Kaplan-Meier-Analyse zum Einsatz. Diese Rechenmethode entwickelten Edward Kaplan und Paul Meier im Jahr 1958 und eignet sich als nichtparametrisches Mittel zur Berechnung von Ereigniszeitanalysen. Es wird die Wahrscheinlichkeit eingeschätzt, dass ein festgelegtes Ereignis innerhalb eines Zeitfensters nicht eintritt. Diese Rechenmethode vergleicht die kumulative Inzidenz von klinischen Ereignissen - in dieser Studie für die Endpunkte Tod, Major Adverse Cardiovascular Events und erneute Revaskularisationsmaßnahmen. Um die Subgruppen in Bezug auf ihren weiteren Krankheitsverlauf zu vergleichen, kam der log-rank-Test zum Einsatz. Entsprechend seiner Entdecker wird dieser statistische Vergleich auch Mantel-Cox-Test genannt.

Um in einem nächsten Schritt festzustellen, ob sich die errechnete Wahrscheinlichkeit und damit der statistische Zusammenhang unabhängig von weiteren adjustierten Kofaktoren darstellt, wurde die Cox-Regressionsanalyse gewählt. Dieses von dem britischen Statistiker David Roxbee Cox entwickelte Mittel eignet sich für die Modellierung von Überlebenszeiten in Ereigniszeitanalysen. Die Rechenmethode basiert auf dem Konzept des Hazardwertes (engl.: hazard: Risiko, Zufall), mit dessen Hilfe eine Aussage über die Wahrscheinlichkeit des Auftretens eines Ereignisses (Tod, Major Adverse Cardiovascular Event und Revaskularisation) getroffen werden kann. Innerhalb dieser sogenannten ,momentanen Neigung zum Zustandswechsel' wird die Hazardfunktion unter Beeinflussung ausgewählter Variablen betrachtet. Um einem Overfitting (engl.: Überanpassung) zu entgehen, müssen die in das Rechenmodell einbezogenen Variablen sorgfältig ausgesucht werden. In dieser Studie wurden für die Regressionsmodelle folgende Confounder aus dem Basisdatensatz gewählt: Geschlecht, Alter bei Einschluss, Body-Mass-Index bei Einschluss, Hypertonie bei Einschluss, Hypercholesterinämie bei Einschluss, Vorhandensein eines Diabetes mellitus bei Einschluss, Tabakabusus bei Einschluss, positive Familienanamnese bei Einschluss, Zustand nach Myokardinfarkt bei Einschluss, Zustand nach Bypass-Operation bei Einschluss, Stent-Typ (DES), Stentlänge, Stentdurchmesser, proximale Lokalisation des Stents und mehr als ein Gefäß über 70\% stenosiert. Der jeweils zu untersuchende psychologische Parameter soll dabei auf seine prognostische Unabhängigkeit überprüft werden. Bei jeder Rechnung wurde auf die Signifikanz (Signifikanzniveau $\alpha=5 \%$ ) des Gesamtmodells geachtet. Die Ergebnisse sind als Hazard Ratio (HR) und deren 95\%-Konfidenzintervall (95\%CI) dargestellt.

In dieser Studie wurden die dokumentierten Endpunkte unter den Aspekten Gesamtmortalität, MACE und Revaskularisation betrachtet und die Patienten je nach Einteilung in die unter 3.5.7 beschriebenen Fraktionen aufgeteilt. So konnte, in Anbetracht der klassifizierten Ergeb- 
nisse der Patientenfragebögen, eine Beziehung zwischen der psychischen Konstitution und dem Wiedereintreten eines erneuten pathologischen Geschehens festgestellt werden.

\subsection{9 Überprüfung der 3. Hypothese: Einfluss der Typ-D-Persönlichkeit}

Ob die Prognose und das erneute Auftreten eines schweren kardialen Ereignisses der Patienten von dem Vorhandensein des Typ-D-Musters bei Einschluss abhängt, soll, wie in 3.5.8 angesprochen, mittels Kaplan-Meier-Analyse und Cox-Regressionsanalyse ermittelt werden. Um zu überprüfen, ob andere, nicht-psychische Parameter ebenfalls eine signifikante Rolle spielen, sollen die beschriebenen Confounder in das Rechenmodell einfließen. Dabei ist nicht nur die dichotome Einteilung in eine Typ-D-Persönlichkeit gegenüber keine Typ-DPersönlichkeit interessant, sondern auch die Analyse der Absolutwerte für negative Affektivität und für soziale Inhibition. Diese beiden Werte lassen sich für die Cox-Regressionsanalyse zu einem Interaktionsterm zusammenfassen, der ebenfalls in das Rechenmodell einfließt. 


\section{ERGEBNISSE}

\subsection{Beschreibung der Stichprobe}

\subsubsection{Soziodemographische Daten der Probanden}

In diese Studie wurden 470 Patienten eingeschlossen, davon 109 (23,2\%) Frauen und 361 (76,8\%) Männer. Zum Zeitpunkt der Implantation des De-novo-Koronarstents waren die Patienten im Durchschnitt 63,7 (SD 9,9) Jahre alt. Die durchschnittliche Größe der Patienten betrug 172,7 (SD 8,4) cm bei einem durchschnittlichen Gewicht von 83,6 (SD 14,1) kg. Daraus ergab sich ein gemittelter Body-Mass-Index (BMI) von 28 (SD 4), wobei die meisten Patienten präadipös waren. Die ausführlichen Angaben zum Gesamtkollektiv zeigt Tabelle 7.

Tabelle 7: Beschreibende Angaben zum Gesamtkollektiv

\begin{tabular}{|l|c|c|c|}
\hline & Gesamt & Männer & Frauen \\
\hline Anzahl & 470 & $361(76,8 \%)$ & $109(23,2 \%)$ \\
\hline Alter (Jahre) (SD) & $63,7(9,9)$ & $63,4(9,9)$ & $64,8(9,7)$ \\
\hline Größe (cm) (SD) & $172,7(8,4)$ & $175,5(6,7)$ & $163,4(6,3)$ \\
\hline Gewicht (kg) (SD) & $83,6(14,1)$ & $86,0(13,3)$ & $75,4(13,7)$ \\
\hline Body-Mass-Index (SD) & $28(4)$ & $27,9(3,7)$ & $28,2(4,8)$ \\
\hline Hypertonie & $380(80,8 \%)$ & $282(78,1 \%)$ & $98(89,9 \%)$ \\
\hline Hypercholesterinämie & $318(67,6 \%)$ & $247(68,4 \%)$ & $71(65,1 \%)$ \\
\hline Diabetes mellitus & $111(23,6 \%)$ & $81(22,4 \%)$ & $30(27,5 \%)$ \\
\hline Tabakabusus & $103(21,9 \%)$ & $81(22,4 \%)$ & $22(20,2 \%)$ \\
\hline Positive Familienanamnese & $181(38,5 \%)$ & $134(37,1 \%)$ & $47(43,1 \%)$ \\
\hline Z.n. Myokardinfarkt & $125(26,6 \%)$ & $100(27,7 \%)$ & $25(22,9 \%)$ \\
\hline Z.n. Bypass-Operation & $57(12,1 \%)$ & $48(13,3 \%)$ & $9(8,2 \%)$ \\
\hline Stenttyp (DES) & $73(15,5 \%)$ & $59(16,3 \%)$ & $14(12,8 \%)$ \\
\hline
\end{tabular}




\begin{tabular}{|l|c|c|c|}
\hline Stentlänge (mm) (SD) & $19,9(9,9)$ & $20,4(10,3)$ & $18,2(8,0)$ \\
\hline Stentdurchmesser (mm) (SD) & $2,9(0,4)$ & $3,0(0,4)$ & $2,9(0,4)$ \\
\hline Proximale Stentlokalisation & $186(39,6 \%)$ & $141(39,1 \%)$ & $45(41,3 \%)$ \\
\hline Mehrgefäßerkrankung & $242(51,5 \%)$ & $193(53,5 \%)$ & $49(44,9 \%)$ \\
\hline
\end{tabular}

\subsubsection{Stentmorphologie}

Bei allen Studienteilnehmern wurde bei Einschluss ein Koronarstent implantiert, wobei die durchschnittliche Stentlänge 19,9 (SD 9,9) mm und der durchschnittliche Stentdurchmesser 2,9 (SD 0,4) mm betrug. Ein Platzieren der Gefäßstütze in den proximalen Bereich der Koronararterie erfolgte bei 186 (39,6\%) Patienten. Bei 73 (15,5\%) Studienteilnehmern wurde ein Drug-Eluting Stent (DES) implantiert, alle anderen bekamen einen Bare Metal Stent (BMS). Eine Zusammenstellung der Daten zu den implantierten Stents zeigt Tabelle 7.

\subsubsection{Kardiologische Risikofaktoren und Vorerkrankungen der Studienteilnehmer}

Der Basisdatensatz, der zu Beginn der Studie angelegt wurde, enthält die klassischen kardialen Risikofaktoren sowie einige Vorerkrankungen der Studienteilnehmer. Ein Diabetes mellitus lag bei $111(23,6 \%)$, eine Hypercholesterinämie bei 318 Patienten (67,6\%) vor. Des Weiteren wiesen 380 Studienteilnehmer (80,8\%) eine arterielle Hypertonie auf. Zum Zeitpunkt der Befragung rauchten 103 Patienten $(21,9 \%)$ und 181 (38,5\%) gaben eine positive Familienanamnese an.

Eine Bypass-Operation vor Studieneinschluss wurde an 57 Patienten $(12,1 \%)$ durchgeführt, bereits 125 (26,6\%) erlitten in ihrer Vorgeschichte einen Myokardinfarkt. Zum Zeitpunkt des Einschlusses lag bei 242 Patienten (51,5\%) eine Koronargefäßstenose über 70\% in mehreren Gefäßen vor. Eine Übersicht über die Vorerkrankungen der Studienteilnehmer zeigt Tabelle 7.

\subsubsection{Beantwortung der Fragebögen bei Aufnahme}

Zu Beginn der Studie hatten alle 470 eingeschlossenen Patienten einen HADS- und 465 von ihnen $(98,9 \%)$ auch einen DS14-Fragebogen ausgefüllt. 


\subsubsection{Ergebnisse der HADS- und DS14-Fragebögen bei Einschluss}

Nach statistischer Auswertung der HADS-Fragebögen ergab sich für die Angst ein Mittelwert von 6,8 (SD 3,7) und für die Depressivität ein Mittelwert von 5,1 (SD 3,4). Im Durchschnitt wurde gemäß DS14 bei der negativen Affektivität ein Wert von 10,6 (SD 5,7) ermittelt und für die soziale Inhibition ein Wert von 9,2 (SD 5,7). Eine Typ-D-Persönlichkeit zeigte sich bei 144 Studienteilnehmern (31\%). Eine Übersicht der Ergebnisse der Fragebögen bei Einschluss zeigt Tabelle 8 .

Tabelle 8: Mittelwerte der psychischen Parameter bei Einschluss

\begin{tabular}{|l|c|c|c|}
\hline Psychischer Parameter & Mittelwert & Standardabweichung & N \\
\hline Angst & 6,8 & 3,7 & 470 \\
Depressivität & 5,1 & 3,4 & 470 \\
Negative Affektivität & 10,6 & 5,7 & 465 \\
Soziale Inhibition & 9,2 & 5,7 & 465 \\
\hline
\end{tabular}

\subsubsection{Häufigkeiten der Endpunkte innerhalb des Nachbeobachtungszeitraums}

Bei 462 (98,3\%) Patienten konnte eine vollständige Nachbeobachtung durchgeführt werden. Innerhalb des 5-jährigen Nachbeobachtungszeitraums kam es zu insgesamt 174 Endpunkten, die kategorisiert und mit Datum versehen aufgenommen wurden. $\mathrm{Zu}$ einem erneuten Implantieren eines Koronarstents innerhalb dieser Subgruppe kam es bei 72 Patienten $(41,4 \%)$, eine PCI ohne Stent war bei 9 Patienten (5,2\%) indiziert. 20 Patienten erlitten einen Myokardinfarkt nach PCI, was 11,5\% der Endpunkte ausmachte. Zu einer aortokoronaren BypassOperation kam es bei 16 Patienten (9,2\%), zu einem apoplektischen Insult 13 (7,5\%) Mal und 44 Patienten verstarben innerhalb des Nachbeobachtungszeitraums, was 25,3\% aller Endpunkte ausmachte. In dieser Studie wurden die Endpunkte in Kategorien zusammengefasst und wie in der Literatur üblich, unter den Aspekten Gesamtmortalität ( $\mathrm{N}=44)$, Major Adverse Cardiovascular Events ( $\mathrm{N}=73)$ und Revaskularisation $(\mathrm{N}=87)$ analysiert (vgl. Tabelle 6). Kam es bei einem Patienten innerhalb des Nachbeobachtungszeitraums zu mehreren klinischen Ereignissen unterschiedlicher Kategorie (z.B. MACE und Revaskularisation) gingen beide Endpunkte getrennt voneinander in die entsprechenden Berechnungen ein. Bei klinischen Ereignissen der gleichen Kategorie (z.B. mehr als eine PCI im Nachbeobachtungszeitraum) 
wurde der erste Endpunkt für die Analyse verwendet. Eine Übersicht über die Verteilung der Endpunkte zeigt Abbildung 1.

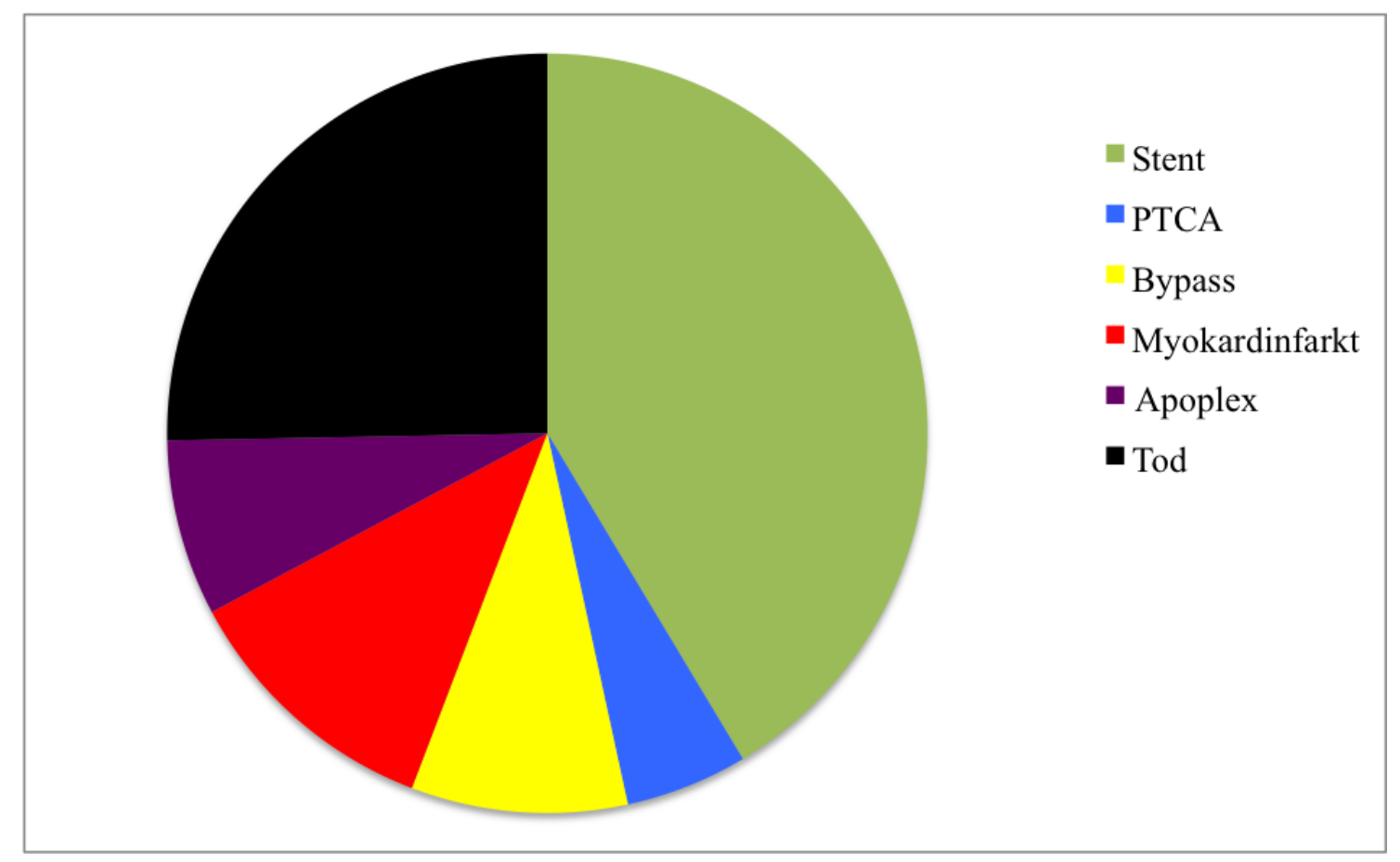

Abb. 1: Verteilung der aufgenommen Endpunkte innerhalb des 5-jährigen Nachbeobachtungszeitraums nach PCI

\section{2 Überprüfen der 1. Hypothese: Prognostischer Einfluss der Depressivität}

Der Kolmogorow-Smirnow-Test (vgl. 3.5.7) zeigte, dass die HADS-Depressions-Werte nicht normal verteilt sind (Kolmogorow-Smirnow-Test: $\mathrm{p}<0,001$ ). Die nicht unübliche schiefe Verteilung der HADS-D-Fragebogenergebnisse innerhalb der Patientenkohorte machte eine Transformation der Studienteilnehmer in Quartile sinnvoll. Diese sollen in Bezug auf die festgelegten Endpunkte auf signifikante Unterschiede untersucht werden, wobei die Zugehörigkeit zum vierten Quartil (HADS-D $\geq 8$ ) in Abgrenzung zu den zusammengefassten unteren drei Quartilen mutmaßlich eine klinische Auffälligkeit bedeutet und daher bei Analysen der Prognose von Interesse war. Diese Unterteilung entspricht gleichzeitig laut Literatur dem wohl trennschärfsten Grenzwert (Bjelland et al. 2002). Nach einem Gruppenvergleich soll die Depressivität auf die Prognose hinsichtlich der bereits benannten Endpunkte Gesamtmortalität, Major Adverse Cardiovascular Events und Revaskularisation untersucht werden. Die Verteilung der HADS-D-Werte zeigt Abbildung 2. 


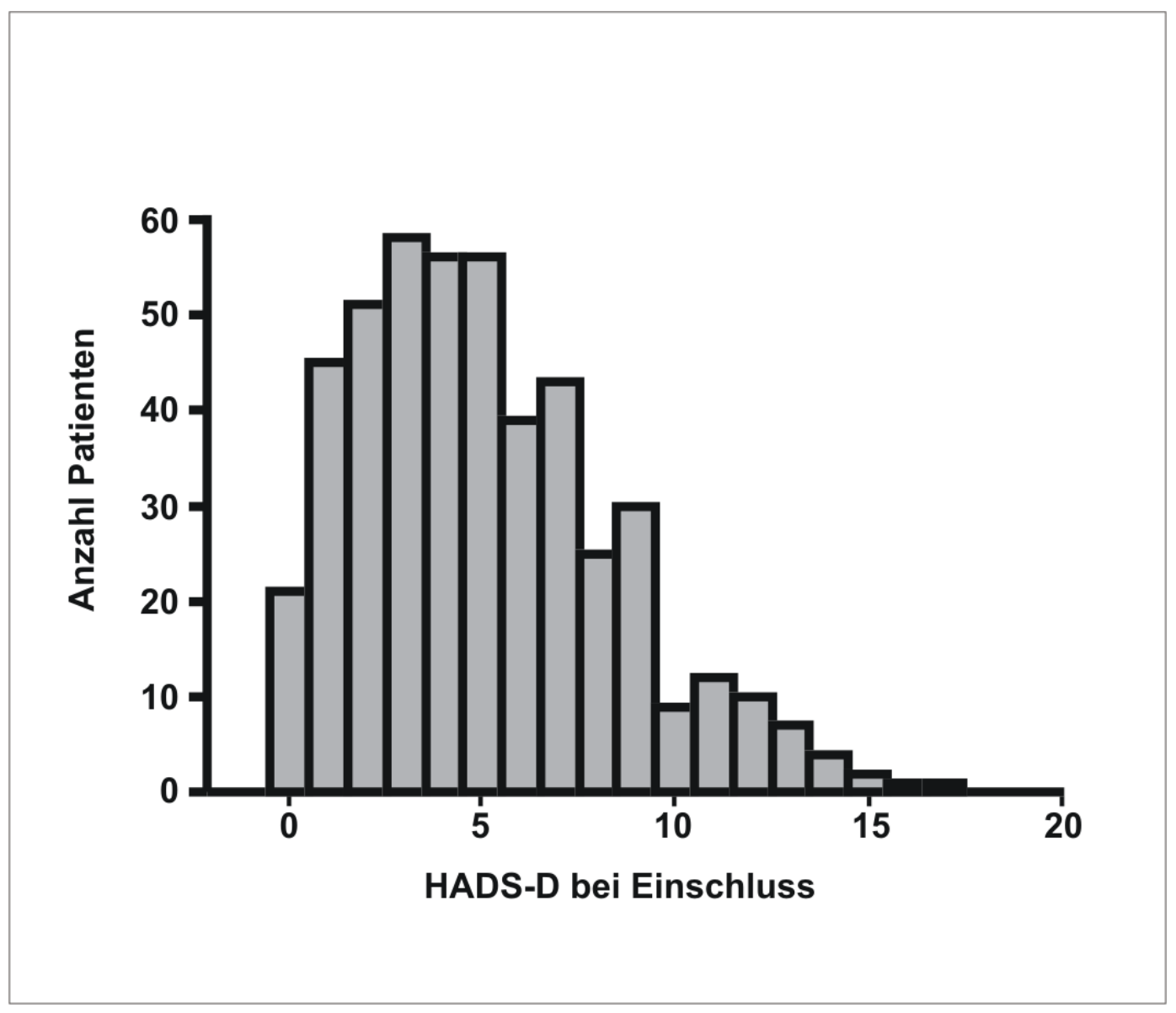

Abb. 2: Hospital Anxiety and Depression Scale-Werte für Depressivität bei Einschluss

\subsubsection{Vergleich der Subgruppen mit und ohne Depressivität}

Bei Einschluss in die Studie füllten alle 470 Patienten den HADS-D-Fragebogen aus. Ein Gruppenvergleich der Patienten mit und ohne Depressivität in Bezug auf den Basisdatensatz zeigte beim Parameter „Alter bei Einschluss“ einen signifikanten Unterschied. Die Studienteilnehmer mit einem HADS-D-positiven Ergebnis waren im Durchschnitt 61,2 (SD 10,4) Jahre alt. Für die Patienten mit HADS-D-negativem Ergebnis ergab sich ein gemittelter Alterswert von 64,4 (SD 9,6), p=0,003. Alle weiteren Daten, einschließlich der Endpunkte Gesamtmortalität $(p=0,561)$ MACE $(p=1,0)$ und Revaskularisation $(p=0,886)$ ergaben keine signifikanten Unterschiede. Die ausführlichen Ergebnisse dieses Gruppenvergleichs finden sich in der Anhangstabelle A.6.

\subsubsection{Testung der 1. Hypothese: Depressivität hat keinen Effekt auf das Überleben}

Nach der Einteilung in die HADS-D-Quartile wurde überprüft, ob es bei Betrachtung der Gesamtmortalität einen signifikanten Unterschied zwischen den Gruppen gibt. Vergleiche des vierten Quartils (11 Todesfälle $=11,2 \%)$ mit dem ersten ( 8 Todesfälle $=6,9 \%, p=0,267)$, dem 
zweiten (14 Todesfälle $=12,3 \%, p=0,812)$ und dem dritten $(11$ Todesfälle=8,2\%, p=0,439) zeigten keine signifikanten Unterschiede. Der Quartilenvergleich mittels Chi-Quadrat-Test über alle vier Gruppen ergab einen p-Wert von 0,469 und konnte daher den angenommenen signifikanten Unterschied bezüglich der Mortalitätsrate innerhalb der 5 Jahre nach PCI ebenfalls nicht bestätigen (vgl. Abb. 3).

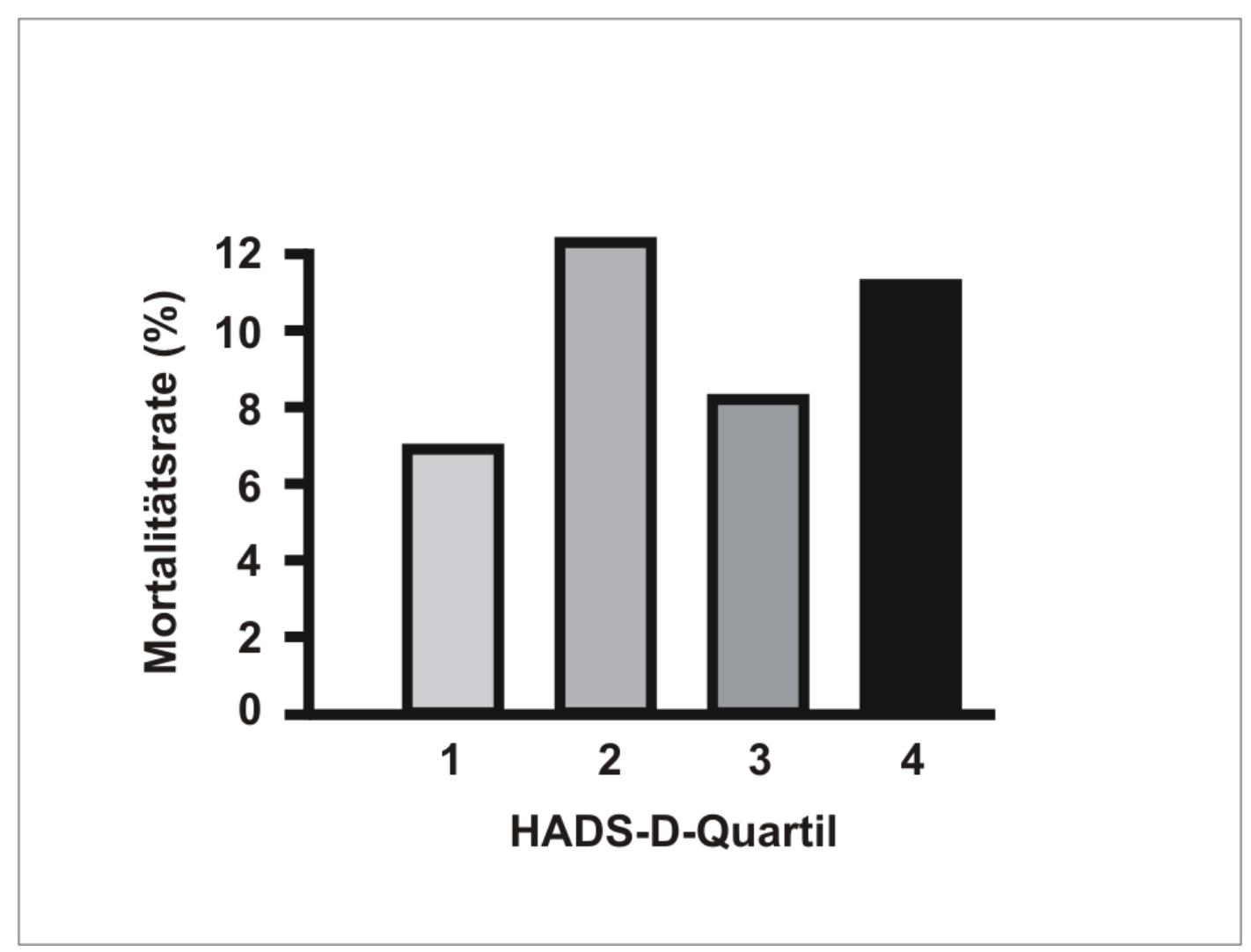

Abb. 3: Kein signifikanter Unterschied $(\mathrm{p}=\mathbf{0 , 4 6 9})$ zwischen den HADS-D-Quartilen bei Betrachtung der Mortalitätsrate innerhalb der 5 Jahre Nachbeobachtung.

Ein Vergleich der unteren drei Quartile untereinander zeigte einen p-Wert von 0,331. Zusammengefasst verstarben von diesen Patienten, die bei Einschluss ein negatives Ergebnis (HADS-D<8) bezüglich der Depressivität hatten, 33 Studienteilnehmer $(8,9 \%)$. Innerhalb der HADS-D-positiven Subgruppe (HADS-D $\geq 8$ ) waren es mit 11 Patienten (10,9\%) etwas mehr. Auch dieser Unterschied ist aber mit einem p-Wert von 0,561 nicht signifikant. Die unadjustierte Kaplan-Meier-Darstellung (vgl. Abb. 4) bestätigt diese Analyse ( $\mathrm{p}=0,451)$, zeigt aber, insbesondere ca. 2 Jahre nach Stentimplantation einen gewissen Trend in die erwartete Richtung. 


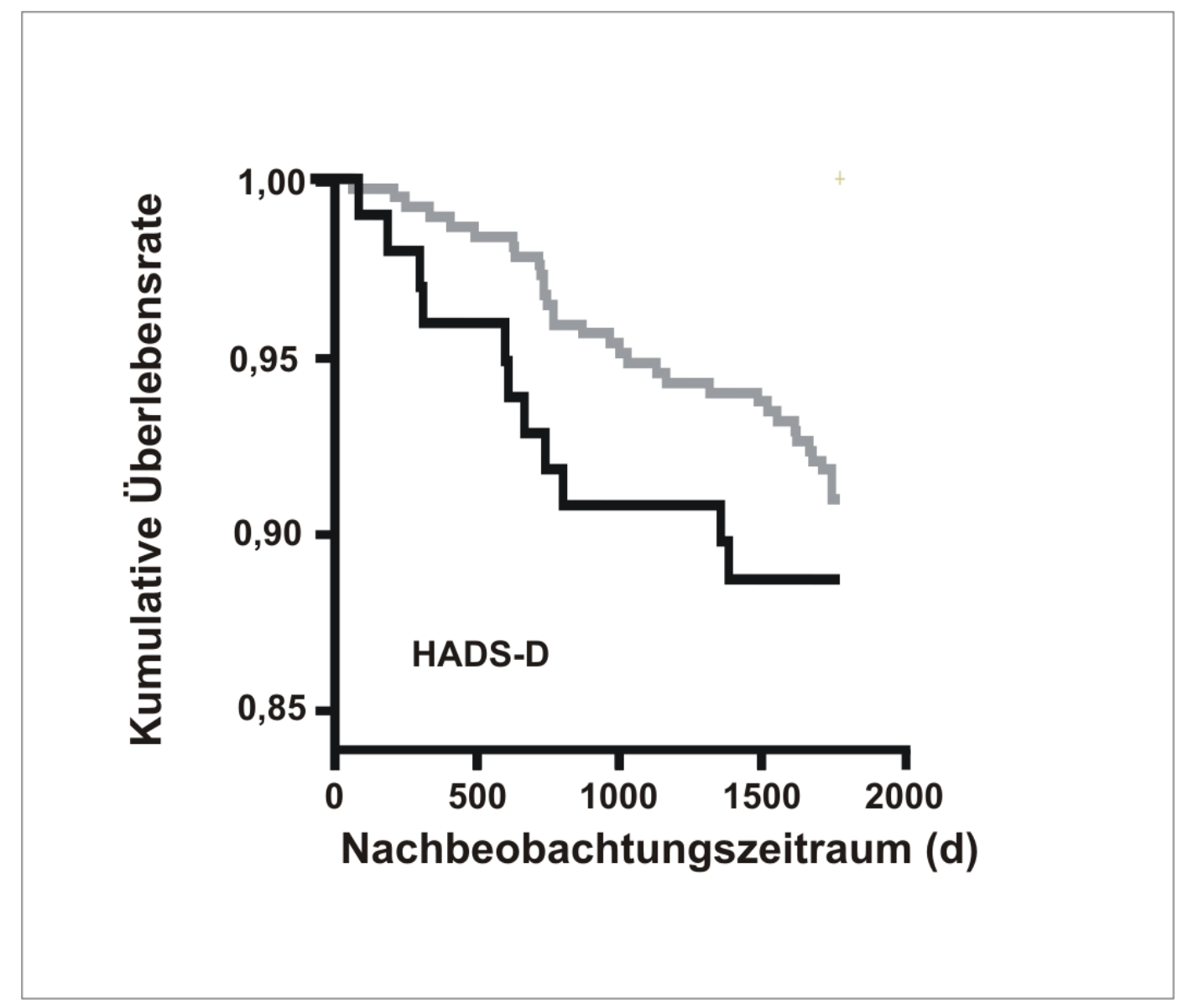

Abb. 4: Kumulative Überlebensrate nach PCI. Dargestellt ist das kumulative Überleben nach initialer Stentimplantation über 5 Jahre Nachbeobachtung. Kein signifikanter Unterschied $(p=0,451)$ der Mortalitätsrate bei HADS-D-positiven Patienten (schwarze Linie), verglichen mit HADS-D-negativen Patienten (graue Linie).

Obwohl das Vorhandensein der Depressivität bei Einschluss keine signifikante Aussagekraft bezüglich der Gesamtmortalität innerhalb von 5 Jahren nach PCI besitzt, zeigte sich deskriptiv ein gewisser Unterschied in die erwartete Richtung bei Patienten im obersten HADS-D Quartil.

\subsubsection{Einflussnahme somatischer Basisdaten auf den Zusammenhang der Gesamtmortalität} mit der Depressivität

Nachdem univariat keine Assoziation der Depressivität mit dem Überleben festgestellt werden konnte, sollte in der nächsten Analyse mittels Multivariatanalyse überprüft werden, ob sich der Effekt durch die Hereinnahme anderer Basisdaten verändert. Hierzu wurde im CoxRegressionsmodell nach Adjustierung aller relevanten, potenziellen Confounder aus dem Basisdatensatz auf Signifikanz getestet. Für das Gesamtmodell ergab sich ein p-Wert von 0,002. 
Mit einer höheren Mortalität assoziiert waren die Confounder Alter bei Einschluss (HR=1,06, 95\% CI=1,02-1,09, p=0,003), Tabakabusus bei Einschluss $(\mathrm{HR}=2,46,95 \% \mathrm{CI}=1,18-5,13$, $\mathrm{p}=0,017)$ und Zustand nach Myokardinfarkt bei Einschluss ( $\mathrm{HR}=1,97,95 \% \mathrm{CI}=1,04-3,73$, $\mathrm{p}=0,036$ ). Für die Variable Body-Mass-Index bei Einschluss ( $\mathrm{HR}=0,9,95 \% \mathrm{CI}=0,82-0,99$, $\mathrm{p}=0,027)$ zeigte sich ein umgekehrter Effekt. Alle anderen Variablen, einschließlich HADS$\mathrm{D} \geq 8$ bei Einschluss $(\mathrm{HR}=1,58,95 \% \mathrm{CI}=0,77-3,22, \mathrm{p}=0,214)$ waren nicht signifikant.

Über die Gesamtmortalitätsrate innerhalb der 5 Jahre nach PCI entschied nicht das Vorhandensein einer Depressivität zum Zeitpunkt des Studienbeginns. Signifikante Ergebnisse zeigten sich nur bei den somatischen Basisdaten der Patienten.

Die Ergebnisse dieser Rechnung zeigt die Anhangstabelle A.1.

\subsubsection{Kein Einfluss der Depressivität auf die Major Adverse Cardiovascular Events}

Verglichen mit dem vierten Quartil (16 MACEs=16\%) zeigten sich im ersten Quartil (17 MACEs $=14,6 \%, p=0,784)$, im zweiten Quartil (21 MACEs $=18,4 \%, p=0,640)$ und im dritten Quartil (19 MACEs=14,2\%, p=0,699) keine signifikanten Unterschiede. Der globale Quartilenvergleich ergab einen p-Wert von 0,808. Wie die Abbildung 5 belegt, besteht zwischen den Quartilen kein Unterschied in Bezug auf die Major Adverse Cardiovascular Events.

Tabelle 9: Verteilung der Major Adverse Cardiovascular Events auf die Depressivitätsquartile

\begin{tabular}{|c|c|c|c|}
\hline $\begin{array}{c}\text { Depressivitäts- } \\
\text { quartil }\end{array}$ & Tod & $\begin{array}{c}\text { Akuter, nicht fata- } \\
\text { ler Myokardinfarkt }\end{array}$ & Apoplex \\
\hline $\mathbf{1}$ & 8 & 5 & 4 \\
\hline $\mathbf{2}$ & 14 & 5 & 4 \\
\hline $\mathbf{3}$ & 11 & 5 & 3 \\
\hline $\mathbf{4}$ & 11 & 5 & 13 \\
\hline Gesamt & 44 & 20 & 4 \\
\hline
\end{tabular}




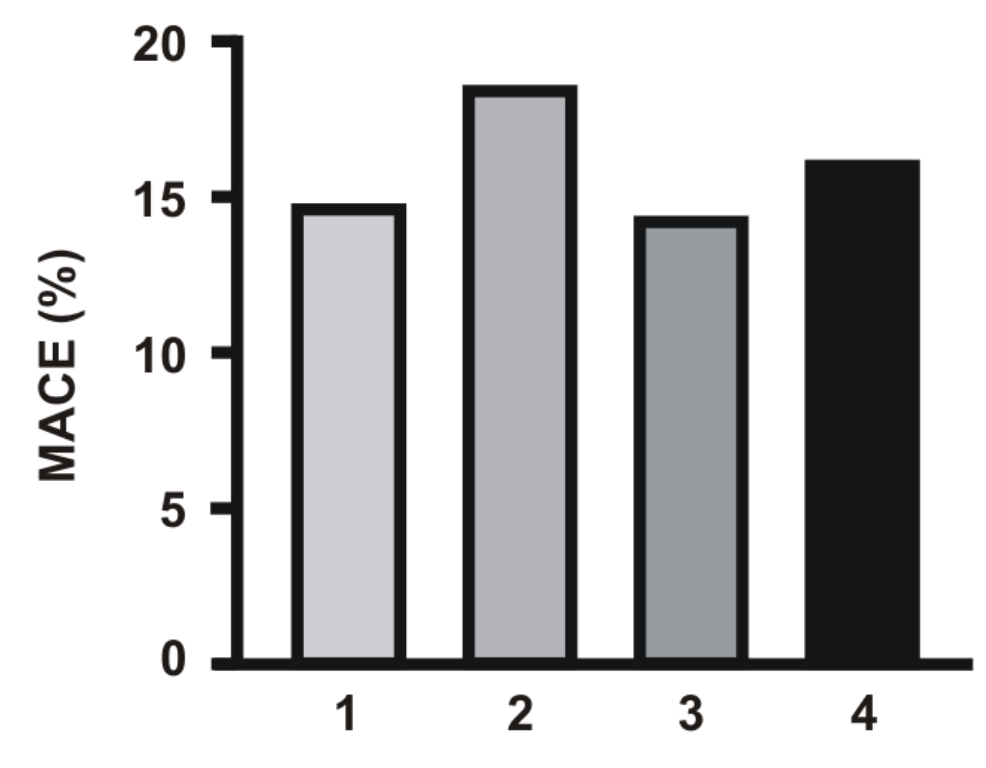

HADS-D-Quartil

Abb. 5: Kein signifikanter Unterschied $(p=0,808)$ zwischen den HADS-D-Quartilen bei Betrachtung der Major Adverse Cardiovascular Events in 5 Jahren Nachbeobachtung.

Ein Vergleich der untersten drei Quartile untereinander ergab einen p-Wert von 0,616. Innerhalb der HADS-D-positiven Subgruppe kam es bei 16 Studienteilnehmern $(15,8 \%)$ zu einem Major Adverse Cardiovascular Event innerhalb des Nachbeobachtungszeitraums. Von Patienten mit negativem HADS-D Ergebnis (entspricht dem zusammengefassten ersten bis dritten Quartil) hatten 57 (15,4\%) Patienten ein erneutes pathologisches Ereignis im Sinne von Apoplex, nicht-fatalem Myokardinfarkt und Tod. Mit einem p-Wert von 1,0 zeigte sich kein signifikanter Unterschied zwischen diesen beiden Subgruppen.

Bei Betrachtung der unadjustierten Kaplan-Meier-Darstellung (vgl. Abb. 6) bestätigt sich, dass das Vorhandensein einer Depressivität keinen Einfluss auf das erneute Auftreten eines Major Adverse Cardiovascular Events hatte $(\mathrm{p}=0,814)$. 


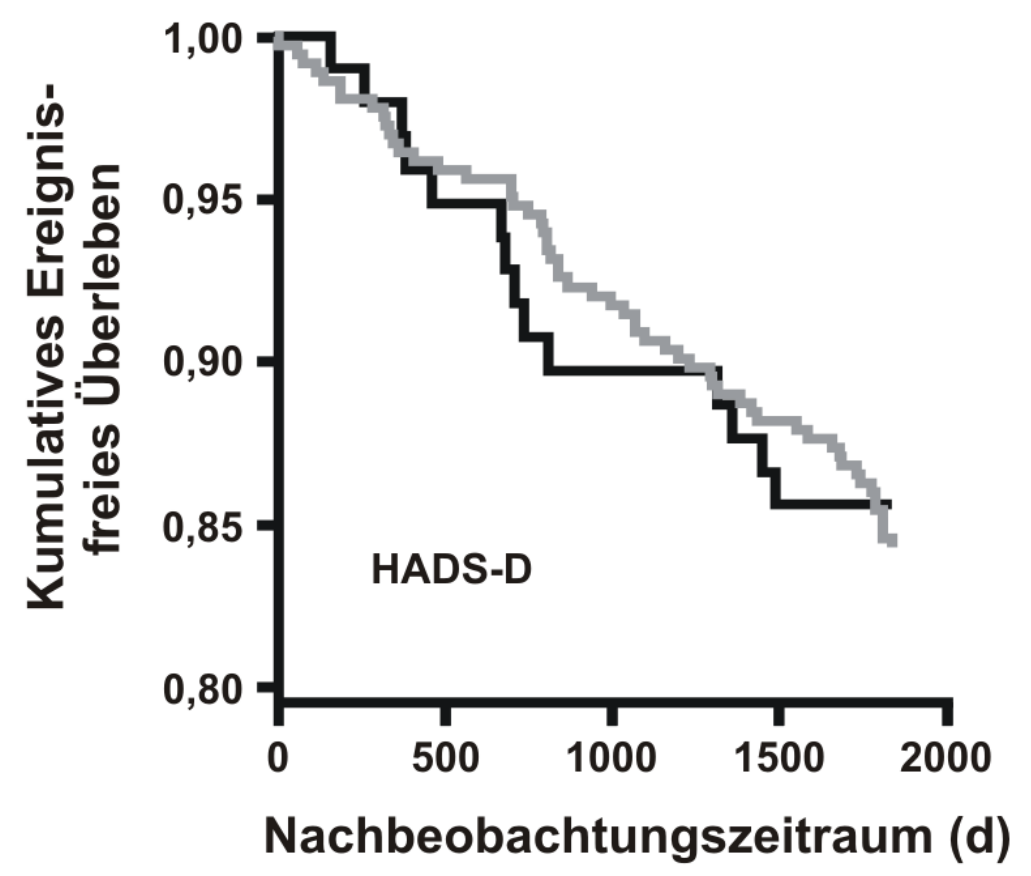

Abb. 6: Kumulative ereignisfreie Überlebensrate nach PCI. Dargestellt ist das kumulative ereignisfreie Überleben nach initialer Stentimplantation über 5 Jahre Nachbeobachtung. Kein signifikanter Unterschied $(p=0,814)$ bei Patienten mit HADS-D-positivem Ergebnis (schwarze Linie), verglichen mit HADSD-negativen Patienten (graue Linie).

\subsubsection{Einflussnahme somatischer Basisdaten auf den Zusammenhang der MACEs mit der} Depressivität

Univariat konnte kein Zusammenhang zwischen der Depressivität und der Rate an Major Averse Cardiovascular Events festgestellt werden. Folglich sollte in einer Multivariatanalyse überprüft werden ob sich durch die Adjustierung anderer Basisdaten der Effekt verändert. $\mathrm{Zu}$ diesem Zweck wurde eine Cox-Regressionsanalyse mit potenziell relevanten Confoundern aus dem Basisdatensatz durchgeführt, wobei sich für das Gesamtmodell ein p-Wert von 0,002 ergab.

Mit einer erhöhten Rate an MACEs gingen die Confounder Alter bei Einschluss (HR=1,03, 95\% CI=1,0-1,06, p=0,016), Tabakabusus bei Einschluss $(\mathrm{HR}=2,69,95 \% \mathrm{CI}=1,5-4,84$, $\mathrm{p}=0,001)$, Zustand nach Myokardinfarkt bei Einschluss ( $\mathrm{HR}=1,78,95 \% \mathrm{CI}=1,07-2,95$, $\mathrm{p}=0,026)$ und Zustand nach Bypass-Operation $(\mathrm{HR}=2,0,95 \% \mathrm{CI}=1,0-3,99, \mathrm{p}=0,048)$ einher. 
Einen Effekt in umgekehrter Richtung hatte die proximale Lokalisation des Stents $(\mathrm{HR}=0,54$, 95\% CI $=0,31-0,95, \mathrm{p}=0,032)$. Alle anderen Variablen, einschließlich HADS-D $\geq 8$ bei Einschluss $(\mathrm{HR}=0,94,95 \% \mathrm{CI}=0,51-1,73, \mathrm{p}=0,84)$ waren nicht signifikant.

Es stellte sich heraus, dass das Vorhandensein einer Depressivität bei Stentintervention keinen Prädiktionswert auf das kumulative ereignisfreie Überleben nach 5 Jahren hatte. Einige somatische Basisdaten zeigten im Gegensatz dazu signifikante Ergebnisse.

Die Ergebnisse dieser Rechnung finden sich im Anhang in der Tabelle A.2.

\subsubsection{Kein Einfluss der Depressivität auf die Revaskularisationsrate}

Wie bei den Endpunkten MACE und Mortalität sollten die HADS-D-Quartile auch mit dem Endpunkt Revaskularisation in Zusammenhang gebracht werden (vgl. Tabelle 10). Ein Vergleich des vierten Quartils (18 Maßnahmen=17,8\%) mit dem ersten (19 Maßnahmen=16,2\%, $\mathrm{p}=0,756$ ), dem zweiten (20 Maßnahmen=17,5\%, p=0,957) und dem dritten (30 Maßnahmen $=21,7 \%, p=0,455)$ zeigte keine signifikanten Unterschiede. Zwischen allen Quartilen erwiesen sich global ebenfalls keine signifikanten Unterschiede $(\mathrm{p}=0,692)$. Abbildung 7 zeigt den Quartilenvergleich in Bezug auf die Revaskularisationsrate.

Tabelle 10: Verteilung der Revaskularisationsmaßnahmen auf die Depressivitätsquartile

\begin{tabular}{|c|c|c|c|}
\hline $\begin{array}{c}\text { Depressivitäts- } \\
\text { quartil }\end{array}$ & $\begin{array}{c}\text { Erneute PCI mit } \\
\text { Stentimplantation }\end{array}$ & PCI & $\begin{array}{c}\text { Koronararterien- } \\
\text { Bypass-Operation }\end{array}$ \\
\hline $\mathbf{1}$ & 14 & 2 & 4 \\
\hline $\mathbf{2}$ & 18 & 1 & 7 \\
\hline $\mathbf{3}$ & 23 & 5 & 3 \\
\hline $\mathbf{4}$ & 17 & 1 & 16 \\
\hline Gesamt & 72 & 9 & 2 \\
\hline
\end{tabular}




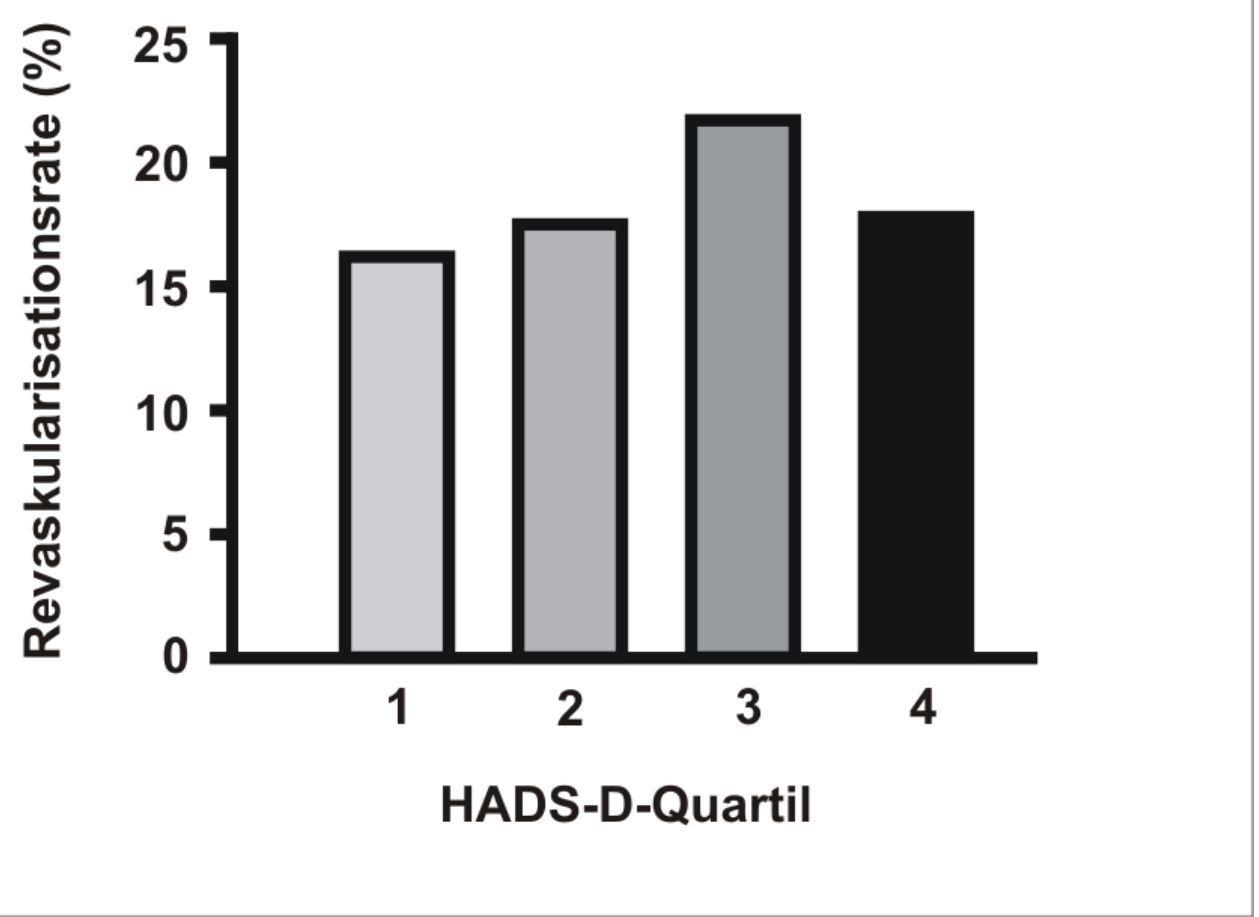

Abb. 7: Kein signifikanter Unterschied (p=0,692) zwischen den HADS-D-Quartilen bei Betrachtung der Revaskularisationsrate innerhalb der 5 Jahre Nachbeobachtung.

Ein Vergleich der drei untersten Quartile untereinander ergab einen p-Wert von 0,495. Zusammengefasst waren in dieser HADS-D-negativen Gruppe 69 (18,7\%) Revaskularisationsmaßnahmen notwendig. Innerhalb der HADS-D-positiven Patientengruppe wurde bei 18 $(17,8 \%)$ Studienteilnehmern eine erneute Revaskularisationsmaßnahme 5 Jahre nach PCI durchgeführt. Dieser Unterschied ist mit einem p-Wert von 0,886 nicht signifikant. Die Kaplan-Meier-Analyse (vgl. Abb. 8) bestätigt dieses Ergebnis ( $p=0,903)$. 


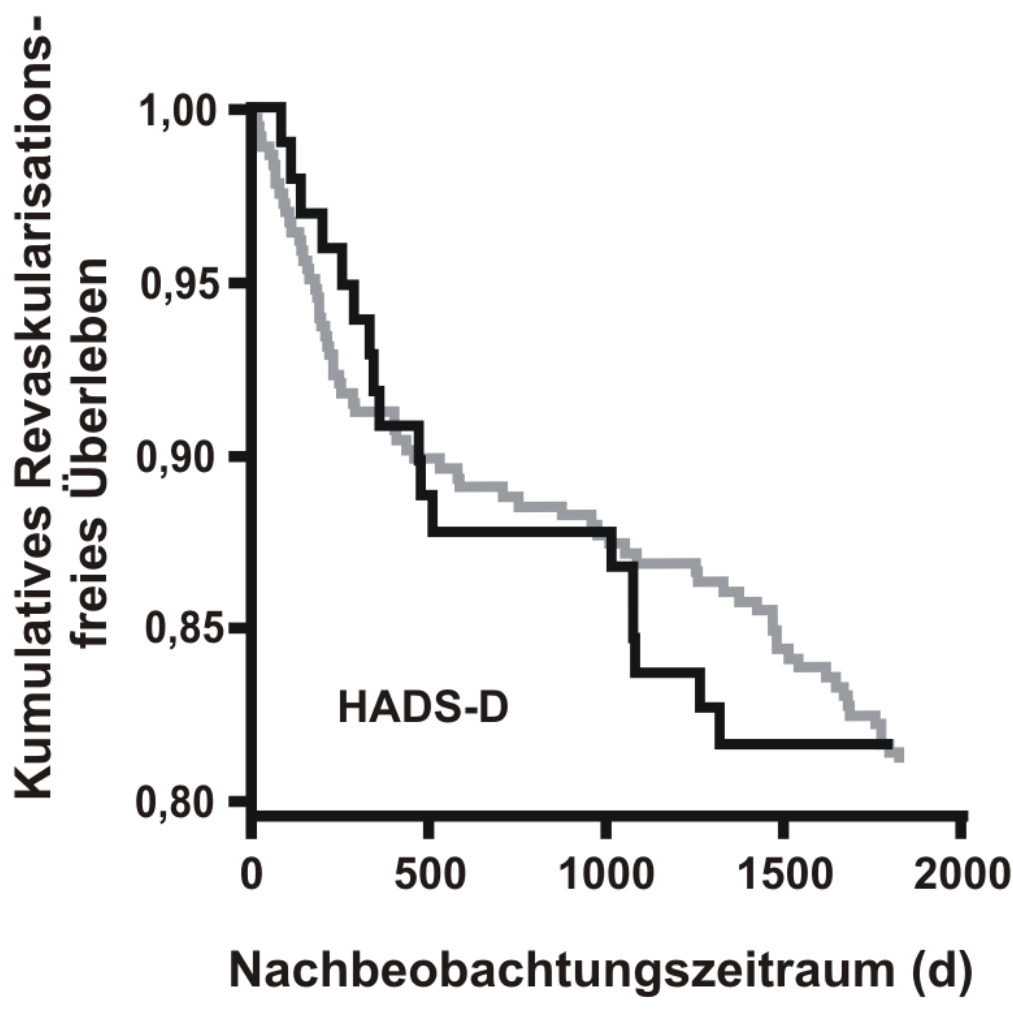

Abb. 8: Revaskularisationsrate nach PCI. Dargestellt ist das kumulative revaskularisationsfreie Überleben nach initialer Stentimplantation über 5 Jahre Nachbeobachtung. Kein signifikanter Unterschied ( $p=0,903)$ der Revaskularisationsrate bei Patienten mit HADS-D $\geq 8$ (schwarze Linie), verglichen mit HADS-D-negativen Patienten (graue Linie).

\subsubsection{Einflussnahme somatischer Basisdaten auf den Zusammenhang der} Revaskularisationsrate mit der Depressivität

Nachdem univariat keine Assoziation der Depressivität auf die Revaskularisationsrate festgestellt werden konnte, sollte in der nächsten Rechnung mittels Multivariatanalyse überprüft werden, ob sich der Effekt nach Hereinnahme anderer Basisdaten verändert. Dafür wurden die gleichen Confounder wie bisher gewählt, jedoch erreichte das Gesamtmodell keinen signifikanten Wert ( $\mathrm{p}=0,303)$, sodass in einem zweiten Schritt neben der Variablen „Depressivitätswert liegt im obersten Quartil“" nur diejenige Kovariate in das Modell eingebracht wurde, die nach univariater Analyse einen signifikanten Wert ergab. Das Gesamtmodell erreichte einen signifikanten p-Wert von 0,048. Die Depressivität erwies sich als irrelevanter Faktor für das Risiko einer erneuten Revaskularisationsmaßnahme ( $\mathrm{HR}=0,93,95 \% \mathrm{CI}=0,55-1,56, \mathrm{p}=0,77)$, während der Zustand nach Bypass-Operation Einfluss nahm (HR=2,02, 95\% CI=1,2-3,4, p=0,008). Die Ergebnisse dieser Rechnung zeigen die Tabellen A.3 und A.4 im Anhang. 
4.2.8 Explorative Zusatzanalyse: Depressivität hat Einfluss auf die Gesamtmortalität im Nachbeobachtungszeitraum von 2 Jahren

In Anbetracht der aktuellen Literatur, in der häufig ein Nachbeobachtungszeitraum von 2 Jahren für die Prognose der Depressivität gewählt wurde sowie der deskriptiven Beobachtung aus der Kaplan-Meier-Kurve, sollte auch diese Studienkohorte auf die Gesamtmortalität innerhalb dieses verkürzten Zeitintervalls untersucht werden. Die statistischen Methoden blieben dabei unverändert.

Innerhalb des Nachbeobachtungszeitraums von 2 Jahren nach Studienbeginn gab es insgesamt 14 Todesfälle. Innerhalb der HADS-D-positiven Patientengruppe verstarben 6 Patienten $(6,1 \%)$. Von den Patienten, die bei Einschluss ein negatives Ergebnis in Bezug auf Depressivität aufwiesen, verstarben 8 Patienten (2,2\%). Der Chi-Quadrat-Test ergab einen signifikanten p-Wert von 0,042. Auch die Kaplan-Meier-Analyse zeigte einen signifikanten Unterschied (p-Wert=0,04) beim Vergleich der Gesamtmortalität zwischen diesen beiden Subgruppen (vgl. Abb. 4 bis zum 730. Tag nach Index-PCI).

4.2.9 Einflussnahme somatischer Basisdaten auf die 2-jährige Gesamtmortalität bei Patienten mit Depressivität

Um den Effekt der Depressivität auf die 2-jährige Mortalitätsrate auf Unabhängigkeit zu prüfen, wurden relevante somatische Basisdaten in einem Cox-Regressionsmodell adjustiert, wobei sich für das Gesamtmodell ein p-Wert von 0,027 ergab. Es zeigte sich für die Depressivität ein unabhängiger Effekt, der mit einer erhöhten Mortalitätsrate einherging $(\mathrm{HR}=4,29$, 95\% CI=1,20-15,39, p=0,025). Während der Confounder „Stentlänge“ ( $\mathrm{HR}=1,06,95 \% \mathrm{CI}=1,0$ 1,11, $\mathrm{p}=0,042)$ gleichgerichtet assoziiert war, zeigte sich für den Body-Mass-Index bei Einschluss $(\mathrm{HR}=0,82,95 \% \mathrm{CI}=0,68-0,99, \mathrm{p}=0,04)$ ein umgekehrter Effekt. Alle anderen Variablen aus dem Basisdatensatz waren nicht signifikant.

Es stellte sich heraus, dass die Depressivität einen signifikanten Einfluss auf die Gesamtmortalität innerhalb eines 2-jährigen Nachbeobachtungszeitraums hatte. Dieser Einfluss erwies sich unabhängig von zahlreichen somatischen Basisdaten.

Die Ergebnisse dieser Rechnung zeigt Tabelle A.5 im Anhang.

Für die anderen Endpunkte war dieser Effekt nicht nachweisbar. Die prognostische Relevanz der Depressivität konnte weder für die Major Adverse Cardiovascular Events (7,9\% versus 
$5,1 \%, \mathrm{p}=0,127)$ noch für die Revaskularisationsmaßnahmen $(11,9 \%$ versus $11,1 \%, \mathrm{p}=0,368)$ innerhalb von 2 Jahren festgestellt werden.

\subsubsection{Verwerfen der 1. Hypothese}

Es ist analysiert worden, ob die Depressivität einen unabhängigen Prädiktor für die Prognose innerhalb von 5 Jahren nach PCI darstellte. Dabei zeigten sich für keinen der oben genannten Endpunkte signifikante Ergebnisse. Für die Gesamtmortalitätsrate ergab sich für 5 Jahre deskriptiv eine etwas höhere Rate für Patienten mit erhöhten HADS-D-Werten. Signifikanten Einfluss auf das weitere Krankheitsgeschehen nach PCI hatten nicht-psychische Faktoren wie Alter, Tabakabusus bei Einschluss und Zustand nach Bypass-Operation. Ein anderes Bild zeigte sich bei der explorativen Untersuchung des verkürzten Nachbeobachtungszeitraums von 2 Jahren. Es ergab sich ein von zahlreichen Kovariaten unabhängiger Zusammenhang der Depressivität auf die Gesamtmortalität. Da die Depressivität als Prädiktionsfaktor innerhalb der 5 Jahre nicht signifikant war und dieser Zeitraum das a priori definierte Zeitintervall dieser Studie darstellt, ist die 1. Hypothese abzulehnen.

\section{3 Überprüfen der 2. Hypothese: Prognostischer Einfluss der Typ-D- Persönlichkeit}

Von den 470 Studienteilnehmern, die bei Einschluss die Fragebögen beantworteten, füllten 465 (98,9\%) die DS14 vollständig aus, wobei die Patienten, die den Fragebogen zu Beginn nicht ausfüllten, für die Betrachtung des Typ-D-Effekts nicht nachbeobachtet wurden. Bei der negativen Affektivität zeigte sich ein durchschnittlicher Wert von 10,6 mit einer Standardabweichung von 5,7. Für die soziale Inhibition ergab sich ein gemittelter Wert von 9,2 mit einer Standardabweichung von 5,7 (vgl. Tabelle 8). Einen Überblick über die Verteilung der NAund SI- Werte sollen die Abbildungen 9 und 10 geben. 144 (31\%) Patienten erreichten in beiden Subskalen Werte von $\geq 10$ und galten somit als Individuen mit Typ-D-Persönlichkeit. 


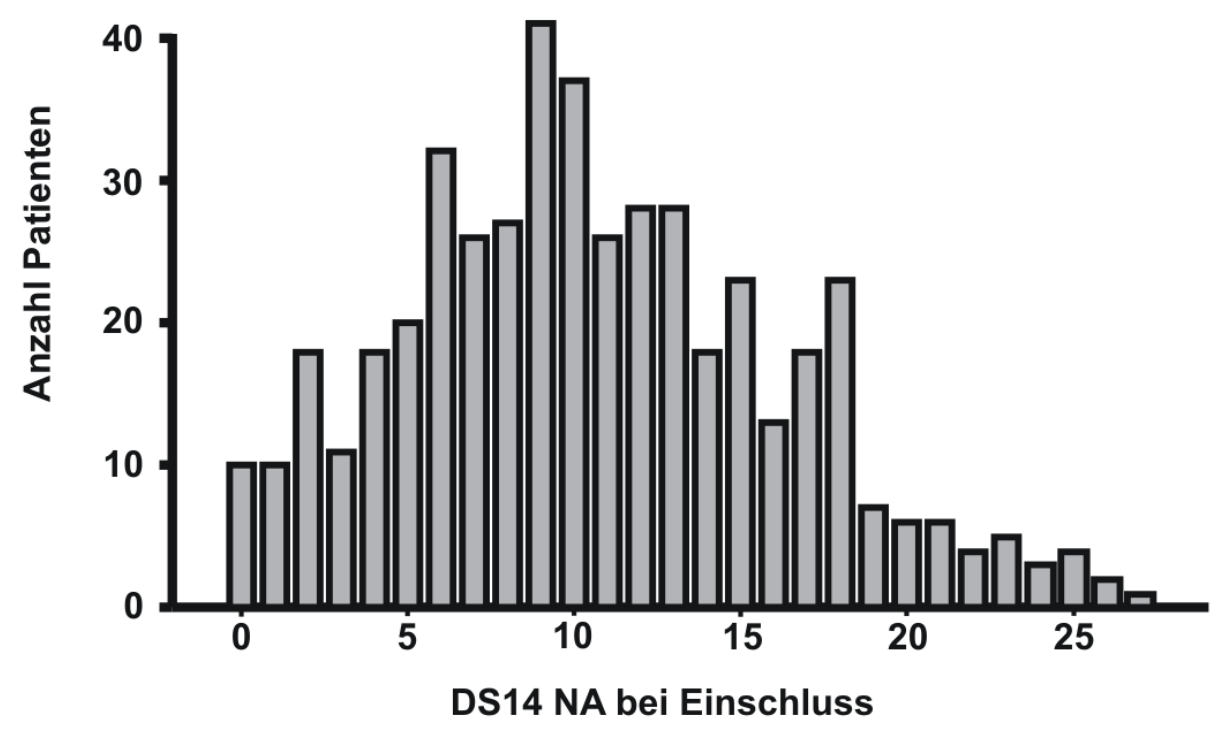

Abb. 9: Verteilung der Werte für negative Affektivität bei Studieneinschluss

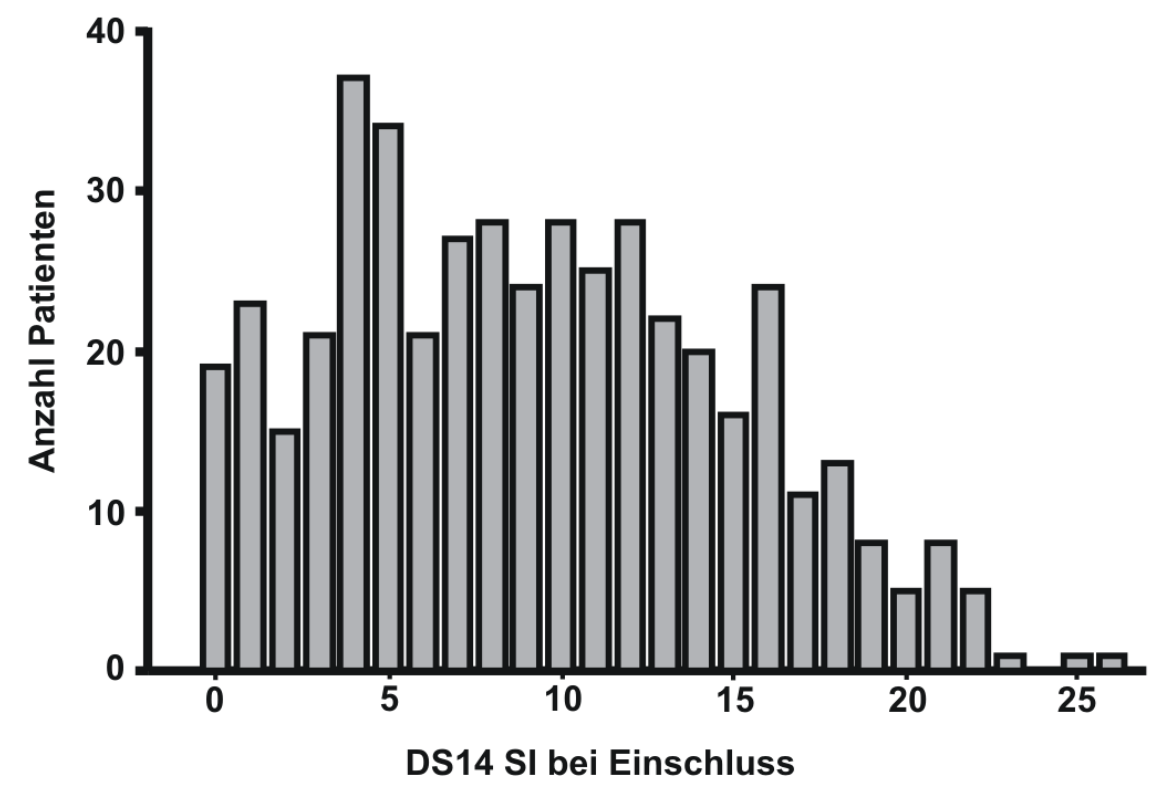

Abb. 10: Verteilung der Werte für soziale Inhibition bei Studieneinschluss 


\subsubsection{Gruppenvergleich der Patienten mit und ohne Typ-D-Persönlichkeit}

Bei dem Gruppenvergleich von Patienten mit und ohne Typ-D-Muster zeigten sich folgende Ergebnisse, wobei sich bei 144 (31\%) Studienteilnehmern eine Typ-D-Persönlichkeit nachweisen ließ. Bei einem Vergleich zeigten sich signifikante Unterschiede in den Basisdaten Alter $(61,9 \pm 10,9$ versus 64,3 \pm 9,2, p=0,005), Hypercholesterinämie bei Einschluss $(76,4 \%$ versus $63,9 \%, \mathrm{p}=0,036)$ und Tabakabusus bei Einschluss $(27,1 \%$ versus $19 \%, \mathrm{p}=0,02)$. Alle anderen Parameter zeigten keinen signifikanten Unterschied.

Obwohl es unerwartet mehr Todesfälle in der Subgruppe ohne Typ-D-Muster (9,3\%) im Vergleich zu den Patienten mit Typ-D-Persönlichkeit (8,3\%) gab, blieb dieser Unterschied statistisch insignifikant $(\mathrm{p}=0,861)$. Bezogen auf die untersuchten Endpunkte Major Adverse Cadiovascular Events $(13,9 \%$ versus $15,9 \%, \mathrm{p}=0,676)$ und Revaskularisation $(19,4 \%$ versus $18,1 \%, p=0,936)$ zeigte sich im Nachbeobachtungszeitraum ebenfalls kein signifikanter Unterschied. Einen ausführlichen Gruppenvergleich in Bezug auf das Vorliegen einer Typ-DPersönlichkeit zeigt Tabelle B.7.

\subsubsection{Testung der 2. Hypothese: Kein Einfluss von Typ-D auf die Gesamtmortalität}

Von den Patienten, die bei Einschluss in die Studie eine Typ-D-Persönlichkeit aufwiesen, verstarben innerhalb des 5-jährigen Beobachtungszeitraums 12 Studienteilnehmer (8,3\%). Von den Studienteilnehmern, die zu Beginn ein negatives Ergebnis in Bezug auf die DS14 zeigten, verstarben 30 (9,3\%) Patienten. Dieser Unterschied war bei einem p-Wert von 0,861 statistisch nicht signifikant.

Bei Betrachtung der unadjustierten Kaplan-Meier-Darstellung (vgl. Abb. 11) bestätigt sich, dass das Vorhandensein einer Typ-D-Persönlichkeit keinen Einfluss auf die Gesamtmortalität hatte $(\mathrm{p}=0,761)$. 


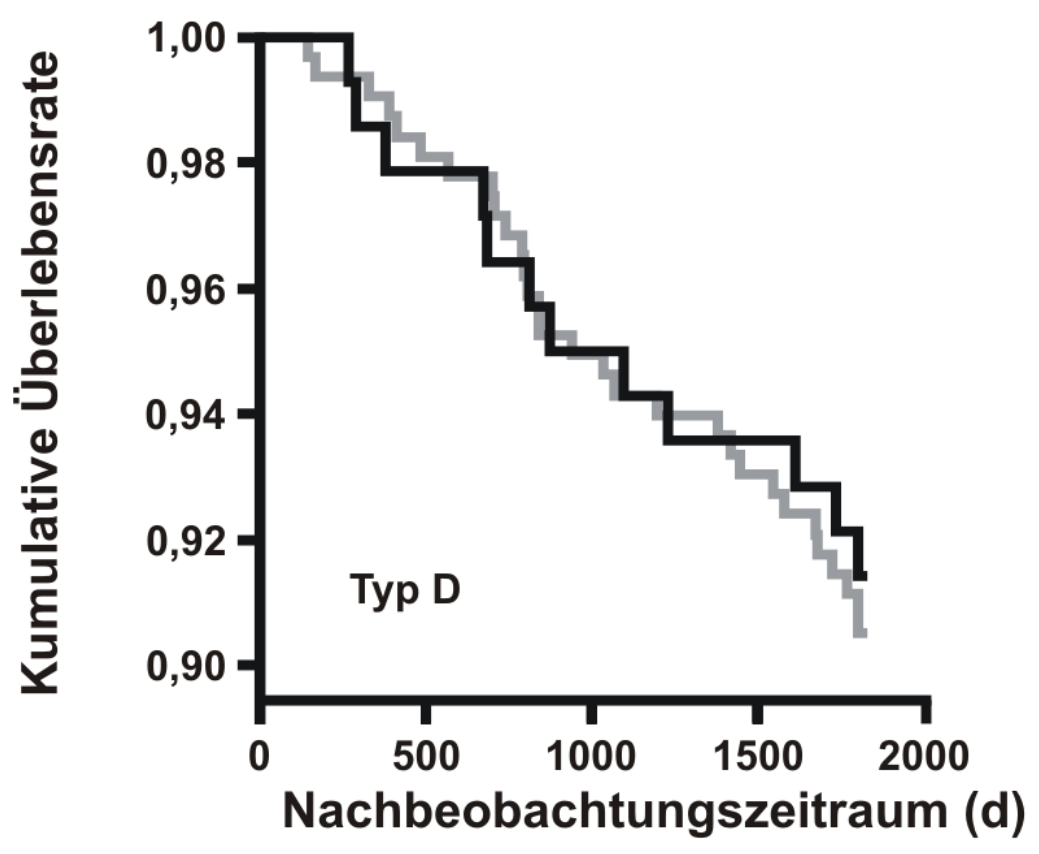

Abb. 11: Kumulative Überlebensrate nach PCI. Dargestellt ist das kumulative Überleben nach initialer Stentimplantation über 5 Jahre Nachbeobachtung. Kein signifikanter Unterschied $(p=0,761)$ der Mortalitätsrate bei Patienten mit Typ-D-Persönlichkeit (schwarze Linie), verglichen mit Patienten ohne Typ-DPersönlichkeit (graue Linie).

\subsubsection{Einflussnahme somatischer Basisdaten auf den Zusammenhang der Gesamtmortalität} mit Typ-D

Als nächstes sollte multivariat überprüft werden, ob sich nach Adjustierung anderer Basisdaten der Typ-D-Effekt auf die Gesamtmortalität verändert. Das Cox-Regressionsmodell zeigte nach Auswahl der gleichen Kovariablen wie bei der Depressivität folgende signifikante Ergebnisse, wobei der p-Wert für das Gesamtmodell bei 0,001 lag. Die Faktoren Alter bei Einschluss $(\mathrm{HR}=1,05,95 \% \mathrm{CI}=1,01-1,09, \mathrm{p}=0,009)$, Tabakabusus bei Einschluss $(\mathrm{HR}=2,42$, 95\% CI=1,13-5,19, $\mathrm{p}=0,023)$ und Zustand nach Myokardinfarkt $(\mathrm{HR}=1,93,95 \% \mathrm{CI}=1,02-3,66$, $\mathrm{p}=0,043)$ gingen mit einem verschlechterten Überleben einher. Einzig der Body-Mass-Index (HR=0,90, 95\%CI=0,82-0,99, p=0,037) zeigte einen gegenteiligen Effekt. Die Typ-DPersönlichkeit $(\mathrm{HR}=1,09,95 \% \mathrm{CI}=0,91-1,31, \mathrm{p}=0,367)$ und alle übrigen Variablen aus dem Basisdatensatz waren nicht signifikant. 
Die Typ-D-Persönlichkeit zeigte auch im multivariaten Modell keinen signifikanten Prädiktionswert für die Mortalität.

Die Ergebnisse dieser Rechnung zeigt Tabelle B.1 im Anhang.

\subsubsection{Kein Einfluss von Typ-D auf das kumulative ereignisfreie Überleben}

Von den Patienten, bei denen zu Beginn der Studie eine Typ-D-Persönlichkeit nachgewiesen werden konnte, erlitten 20 (13,9\%) im Nachverfolgungszeitraum ein schweres kardiovaskuläres Ereignis (MACE). Bei Patienten ohne Typ-D-Persönlichkeit kam es bei 51 (15,9\%) erneut zu einem Major Adverse Cardiovascular Event, wobei bei mehreren Ereignissen das jeweils erste in die Berechnung einging. Ein statistischer Vergleich zeigte bei einem p-Wert von 0,676 keinen signifikanten Unterschied.

Tabelle 11: Verteilung der Major Adverse Cardiovascular Events bei Typ-D

\begin{tabular}{|c|c|c|c|}
\hline & Tod & $\begin{array}{c}\text { Akuter, nicht fata- } \\
\text { ler Myokardinfarkt }\end{array}$ & Apoplex \\
\hline Typ-D & 12 & 7 & 3 \\
\hline Kein Typ-D & 30 & 13 & 10 \\
\hline Gesamt & 42 & 20 & 13 \\
\hline
\end{tabular}

Bei der Betrachtung der unadjustierten Kaplan-Meier-Darstellung (vgl. Abb. 12) bestätigte sich, dass das Vorhandensein einer Typ-D-Persönlichkeit keinen Einfluss auf das erneute Auftreten eines schweren kardialen Events hatte ( $\mathrm{p}=0,535)$. Die Typ-D-Persönlichkeit hatte demnach in dieser Patientenkohorte keinen signifikanten Prädiktionswert bezüglich des Auftretens eines erneuten pathologischen Ereignisses im Sinne von Apoplex, nicht-fataler Myokardinfarkt und Tod. 


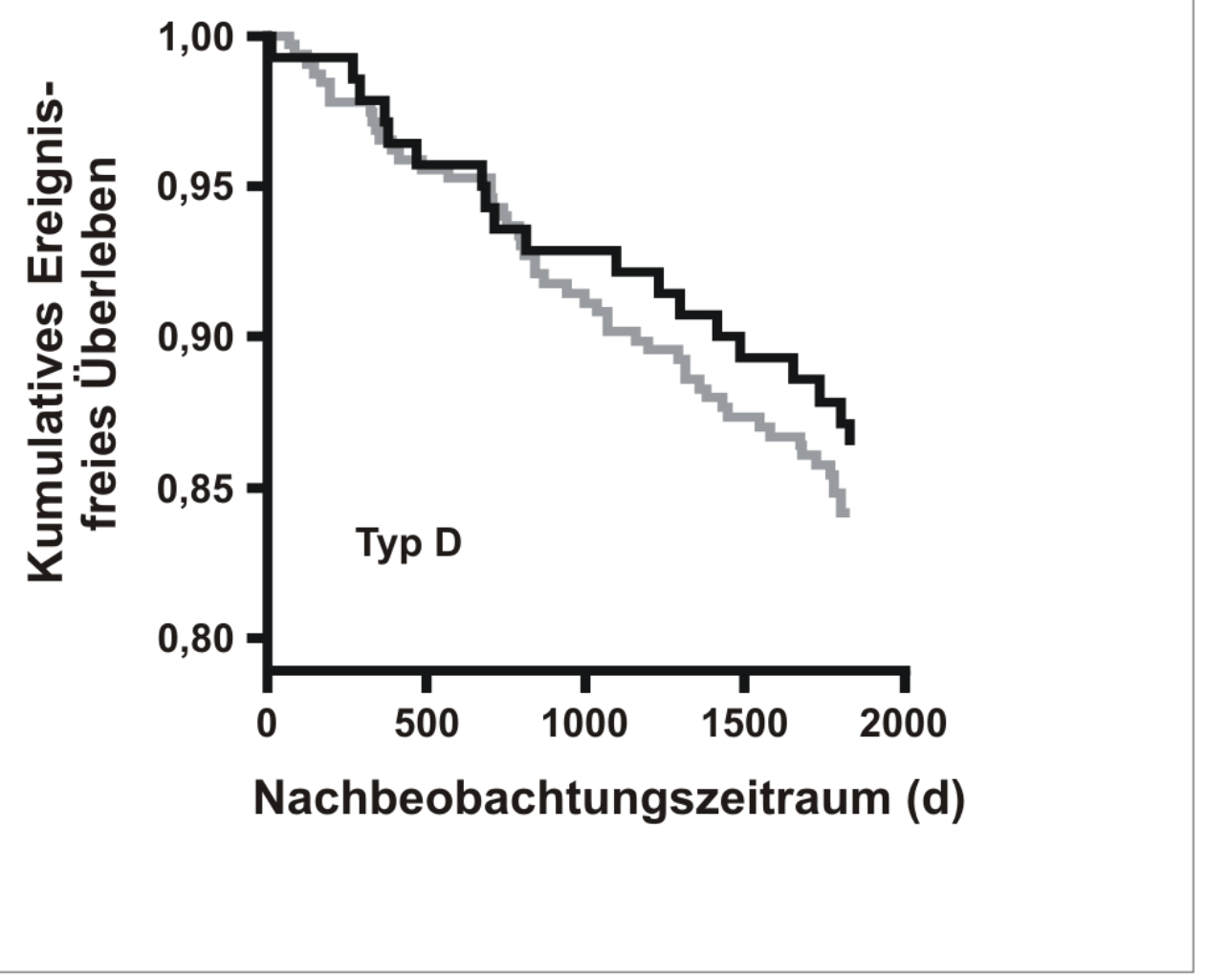

Abb. 12: Kumulative ereignisfreie Überlebensrate nach PCI. Dargestellt ist das kumulative ereignisfreie Überleben nach initialer Stentimplantation über 5 Jahre Nachbeobachtung. Kein signifikanter Unterschied (p=0,535) bei Patienten mit Typ-D-Persönlichkeit (schwarze Linie), verglichen mit Patienten ohne Typ-D-Persönlichkeit (graue Linie).

Zwar findet sich beim Vergleich der Patienten mit und ohne Typ-D-Persönlichkeit in Bezug auf das kumulative ereignisfreie Überleben kein signifikanter Unterschied, dennoch zeigt sich ein gewisser Trend. In der ersten Hälfte des Beobachtungszeitraums lässt sich so gut wie kein Unterschied zwischen den beiden Subgruppen feststellen. In der zweiten Hälfte, also ab ca. 2,5 Jahren, zeigt sich unerwartet eine leicht niedrigere Ereignisrate bei Typ-D-Patienten. Studienteilnehmer, die bei Einschluss eine Typ-D-Persönlichkeit aufgewiesen haben, erlitten im Verlauf geringfügig und statistisch nicht signifikant weniger Ereignisse im Sinne von Apoplex, nicht-fataler Myokardinfarkt und Tod. 
4.3.5 Einflussnahme somatischer Basisdaten auf das kumulative ereignisfreie Überleben bei Typ-D

In einer Cox-Regressionsanalyse sollte überprüft werden, ob nach Adjustierung anderer Basisdaten der Effekt der Typ-D-Persönlichkeit auf die Rate der Major Adverse Cardiovascular Events verändert wird bzw. ob nach der Hereinnahme ein Zusammenhang besteht. Für das Gesamtmodell ergab sich ein signifikanter p-Wert von 0,001 .

Mit einer erhöhten Rate an MACEs waren Alter bei Einschluss (HR=1,03, 95\%CI=1,01-1,06, p=0,019), Tabakabusus bei Einschluss ( $\mathrm{HR}=2,57,95 \% \mathrm{CI}=1,42-4,64, \mathrm{p}=0,002)$, Zustand nach Myokardinfarkt $(\mathrm{HR}=1,77,95 \% \mathrm{CI}=1,07-2,93, \mathrm{p}=0,027)$ und Zustand nach Bypass-Operation $(\mathrm{HR}=1,98,95 \% \mathrm{CI}=1,0-3,91, \mathrm{p}=0,05)$ assoziiert. Einzig die proximale Lokalisation des Stents $(\mathrm{HR}=0,53,95 \% \mathrm{CI}=0,30-0,93, \mathrm{p}=0,028)$ zeigte einen gegenteiligen Effekt. Die Typ-DPersönlichkeit $(\mathrm{HR}=1,05,95 \% \mathrm{CI}=0,88-1,26, \mathrm{p}=0,564)$ und alle übrigen Variablen aus dem Basisdatensatz waren nicht signifikant.

Es zeigte sich, dass die Typ-D-Persönlichkeit auch im multivariaten Modell keine Aussagekraft bezüglich der Wahrscheinlichkeit des Wiederauftretens eines Major Adverse Cardiovascular Events besaß.

Die Ergebnisse dieser Rechnung sind im Anhang der Tabelle B.2 zu entnehmen.

\subsubsection{Kein Einfluss von Typ-D auf die Revaskularisationsrate}

Bei 28 (19,4\%) Patienten mit Typ-D-Persönlichkeit und bei 58 (18,1\%) Patienten ohne TypD-Persönlichkeit war innerhalb der 5 Jahre nach PCI mindestens eine Revaskularisationsmaßnahme notwendig. Auch dieser statistische Vergleich blieb mit einem p-Wert von 0,936 insignifikant.

Tabelle 12: Verteilung der Revaskularisationsmaßnahmen bei Typ-D

\begin{tabular}{|c|c|c|c|}
\hline & $\begin{array}{c}\text { Erneute PCI mit } \\
\text { Stentimplantation }\end{array}$ & PCI & $\begin{array}{c}\text { Koronararterien- } \\
\text { Bypass- } \\
\text { Operation }\end{array}$ \\
\hline Typ-D & 25 & 2 & 5 \\
\hline Kein Typ-D & 47 & 7 & 10 \\
\hline Gesamt & 72 & 7 & 15 \\
\hline
\end{tabular}


Bei der Betrachtung der unadjustierten Kaplan-Meier-Darstellung (vgl. Abb. 13) bestätigte sich, dass das Vorhandensein einer Typ-D-Persönlichkeit keinen Einfluss auf die Revaskularisationsrate hatte $(\mathrm{p}=0,632)$.

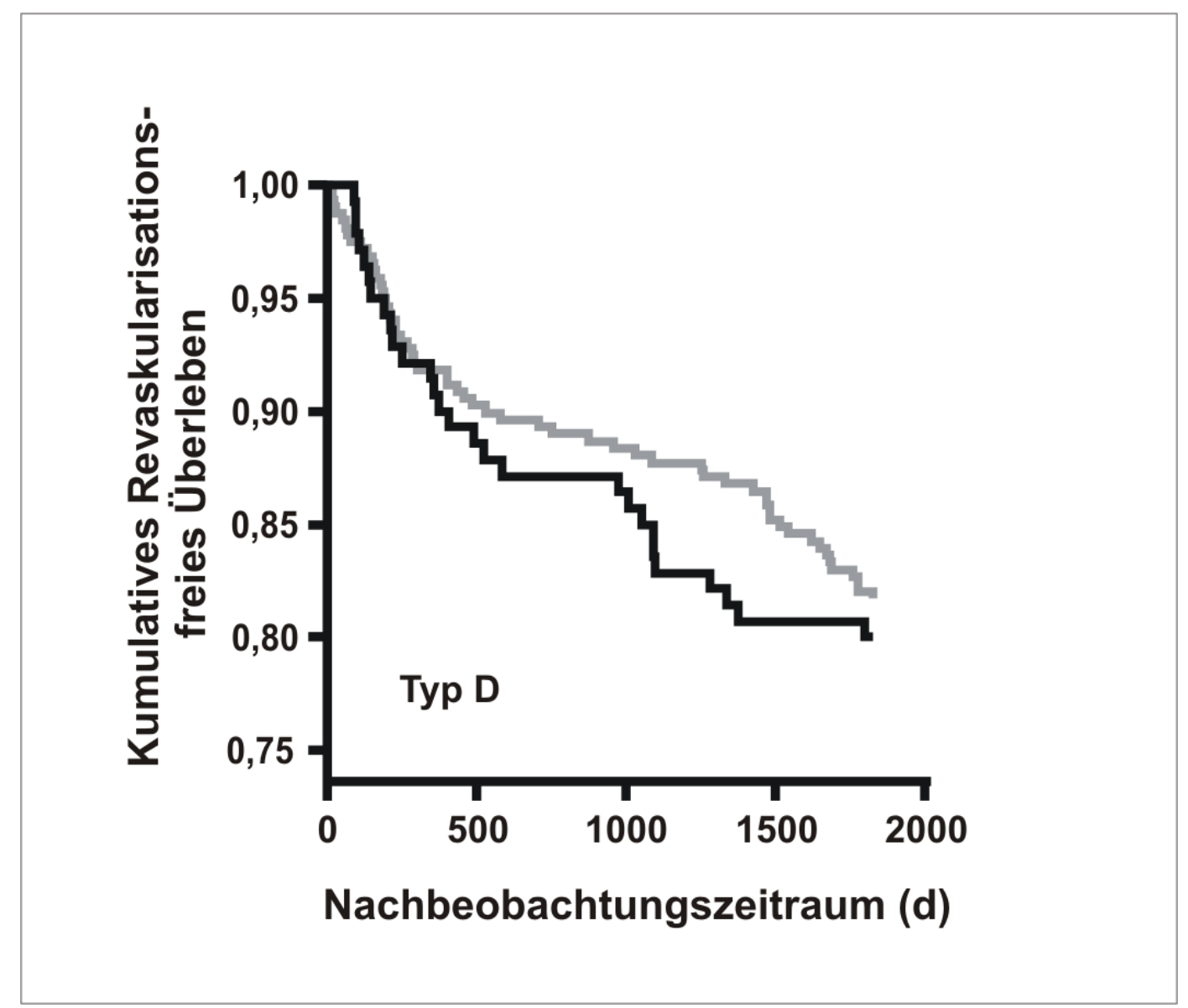

Abb. 13: Revaskularisationsrate nach PCI. Dargestellt ist das kumulative revaskularisationsfreie Überleben nach initialer Stentimplantation über 5 Jahre Nachbeobachtung. Kein signifikanter Unterschied ( $p=0,632)$ der Revaskularisationsrate bei Patienten mit Typ-D-Persönlichkeit (schwarze Linie), verglichen mit Patienten ohne Typ-D-Persönlichkeit (graue Linie).

Beim Vergleich der Revaskularisationsraten beider Subgruppen mit und ohne Typ-DPersönlichkeit zeigte sich ein gewisser Trend innerhalb der 5 Jahre. Innerhalb des ersten Viertels nach Studieneinschluss ergab sich kein Unterschied zwischen Patienten mit und ohne Typ-D-Persönlichkeit. Danach stieg bei Patienten mit Typ-D-Muster die Rate der Revaskularisationsmaßnahmen geringfügig an. 
4.3.7 Einflussnahme somatischer Basisdaten auf den Zusammenhang der Revaskularisationsrate mit Typ-D

In einer weiteren Cox-Regressionsanalyse sollte überprüft werden, ob nach Adjustierung anderer relevanter Basisdaten ein Typ-D-Effekt auf die Rate der Major Adverse Cardiovascular Events besteht. Jedoch erreichte das Gesamtmodell bei einer Auswahl der gleichen Confounder wie bisher keinen signifikanten Wert $(\mathrm{p}=0,319)$. Nach Kontrolle der Confounder ergab sich somit ebenfalls kein Effekt des Typ-D-Musters auf die Revaskularisationsrate. Die Ergebnisse dieser Cox-Regressionsanalyse finden sich im Anhang in der Tabelle B.3.

\subsubsection{Negative Affektivität, soziale Inhibition und ihr Interaktionsterm als Prädiktoren des} Verlaufs

In einem weiteren Cox-Regressionsmodell sollte überprüft werden, ob die Absolutwerte für negative Affektivität und für soziale Inhibition bzw. deren Interaktionsterm eine prognostische Aussage bezüglich der Endpunkte hatten. Bei den zuvor verwendeten Confoundern ergab sich bei Betrachtung der Mortalitätsrate für das Gesamtmodell ein signifikanter p-Wert von 0,033. Anhand der Rohwerte für negative Affektivität ( $\mathrm{HR}=1,02,95 \% \mathrm{CI}=0,91-1,15$, $\mathrm{p}=0,697)$, für soziale Inhibition $(\mathrm{HR}=1,0,95 \% \mathrm{CI}=0,89-1,13, \mathrm{p}=0,963)$ und anhand des Interaktionsterms aus DS14-NA*DS14-SI (HR=1,0, 95\%CI=0,99-1,01, p=0,976) konnte man abermals keinen Einfluss der DS14-Skalenwerte auf die Gesamtmortalität nachweisen. Signifikante Einflussgrößen waren in diesem Rechenmodell Alter bei Einschluss $(\mathrm{HR}=1,05$, 95\% CI=1,01-1,09, p=0,011), Tabakabusus bei Einschluss ( $\mathrm{HR}=2,22,95 \% \mathrm{CI}=1,02-4,86$, $\mathrm{p}=0,045)$ und Body-Mass-Index $(\mathrm{HR}=0,9,95 \% \mathrm{CI}=0,82-1,0, \mathrm{p}=0,043)$. Alle anderen Variablen aus dem Basisdatensatz waren nicht signifikant.

Die Rohwerte der negativen Affektivität und der sozialen Inhibition sowie deren Interaktionsterm hatten in der multivariaten Analyse keinen signifikanten Effekt auf die Mortalitätsrate.

Die Ergebnisse dieser Rechnung sind im Anhang der Tabelle B.4 zu entnehmen.

Ein ähnliches Bild zeigte sich bei gleicher Cox-Regressionsanalyse, dieses Mal mit Major Adverse Cardiovascular Events als Endpunkt. Das Gesamtmodell ergab einen p-Wert von 0,003. Nach Abschluss dieser Rechnung und Adjustierung für die oben genannten Confounder zeigten sich für die Absolutwerte der negativen Affektivität $(\mathrm{HR}=1,04,95 \% \mathrm{CI}=0,96-1,13$, $\mathrm{p}=0,359)$ und der sozialen Inhibition $(\mathrm{HR}=0,95,95 \% \mathrm{CI}=0,86-1,04, \mathrm{p}=0,282)$ wie auch für den 
Interaktionsterm aus DS14-NA*DS14-SI $(\mathrm{HR}=1,0,95 \% \mathrm{CI}=0,99-1,01, \mathrm{p}=0,756)$ kein signifikanter Vorhersageeffekt. Einen Effekt auf den weiteren Verlauf hatten die Variablen Alter bei Einschluss ( $\mathrm{HR}=1,04,95 \% \mathrm{CI}=1,01-1,07, \mathrm{p}=0,01)$, Tabakabusus bei Einschluss $(\mathrm{HR}=2,52$, 95\% CI=1,37-4,62, p=0,003), Zustand nach Myokardinfarkt $(\mathrm{HR}=1,79,95 \% \mathrm{CI}=1,06-3,02$, $\mathrm{p}=0,03)$, Zustand nach Bypass-Operation $(\mathrm{HR}=2,24,95 \% \mathrm{CI}=1,12-4,47, \mathrm{p}=0,023)$ und proximale Lokalisation des Stents $(\mathrm{HR}=0,52,95 \% \mathrm{CI}=0,29-0,92, \mathrm{p}=0,025)$. Alle anderen Variablen waren nicht signifikant.

Die Rohwerte der negativen Affektivität und der sozialen Inhibition sowie deren Interaktionsterm hatten keinen signifikanten Effekt auf die Major Adverse Cardiovascular Events.

Die Ergebnisse dieser Rechnung sind im Anhang der Tabelle B.5 zu entnehmen.

Das Cox-Regressionsmodell mit der Revaskularisation als Endpunkt erreichte keine Signifikanz, womit sich ebenfalls kein Vorhersageeffekt der DS-14-Subskalen bzw. ihres Interaktionsterms auf die Revaskularisationsrate ergab (vgl. Tabelle B.6 im Anhang).

\subsubsection{Verwerfen der 2. Hypothese}

Es wurde überprüft, ob das Vorhandensein einer Typ-D-Persönlichkeit zum Zeitpunkt des Implantierens eines Koronarstents einen unabhängigen Prädiktor für den weiteren Krankheitsverlauf darstellt. Dabei zeigten sich für keinen der aufgenommenen Endpunkte signifikante Ergebnisse für den Nachbeobachtungszeitraum von 5 Jahren, auch dann nicht, wenn anstelle der Typ-D-Persönlichkeit die Rohwerte der negativen Affektivität und der sozialen Inhibition sowie deren Interaktionsterm betrachtet wurden. Da ein Effekt der Typ-DPersönlichkeit auf die untersuchten Endpunkte in dieser Studienkohorte nicht nachgewiesen werden konnte, ist die 2. Hypothese zu verwerfen.

\section{4 Überprüfen der 3. Hypothese: Prognostischer Einfluss der Angst}

Es zeigte sich nach Anwendung des Kolmogorow-Smirnow-Tests (vgl. 3.5.7), dass die HADS-Angst-Werte nicht normal verteilt sind (Kolmogorow-Smirnow-Test: $\mathrm{p}=0,002$ ). Wegen der nicht ungewöhnlichen schiefen Verteilung der Fragebogenergebnisse innerhalb dieser Studienkohorte erfolgte eine Transformation der Patientenkohorte in Quartile. In Bezug auf die festgelegten Endpunkte wurden diese Quartile im weiteren Verlauf auf signifikante Unterschiede untersucht. Der Vergleich des obersten Angstquartils (HADS-A $\geq 10$ ) mit den zusammengefassten unteren drei Quartilen bedeutet mutmaßlich eine Unterscheidung zwischen kli- 
nischer Auffälligkeit und Normalbefund und soll bei den Analysen der Prognose von Interesse sein, wobei in der Literatur Grenzwerte zwischen HADS-A $\geq 8$ und $\geq 11$ zu finden sind. Die Verteilung der HADS-A-Werte bei Einschluss zeigt Abbildung 14.

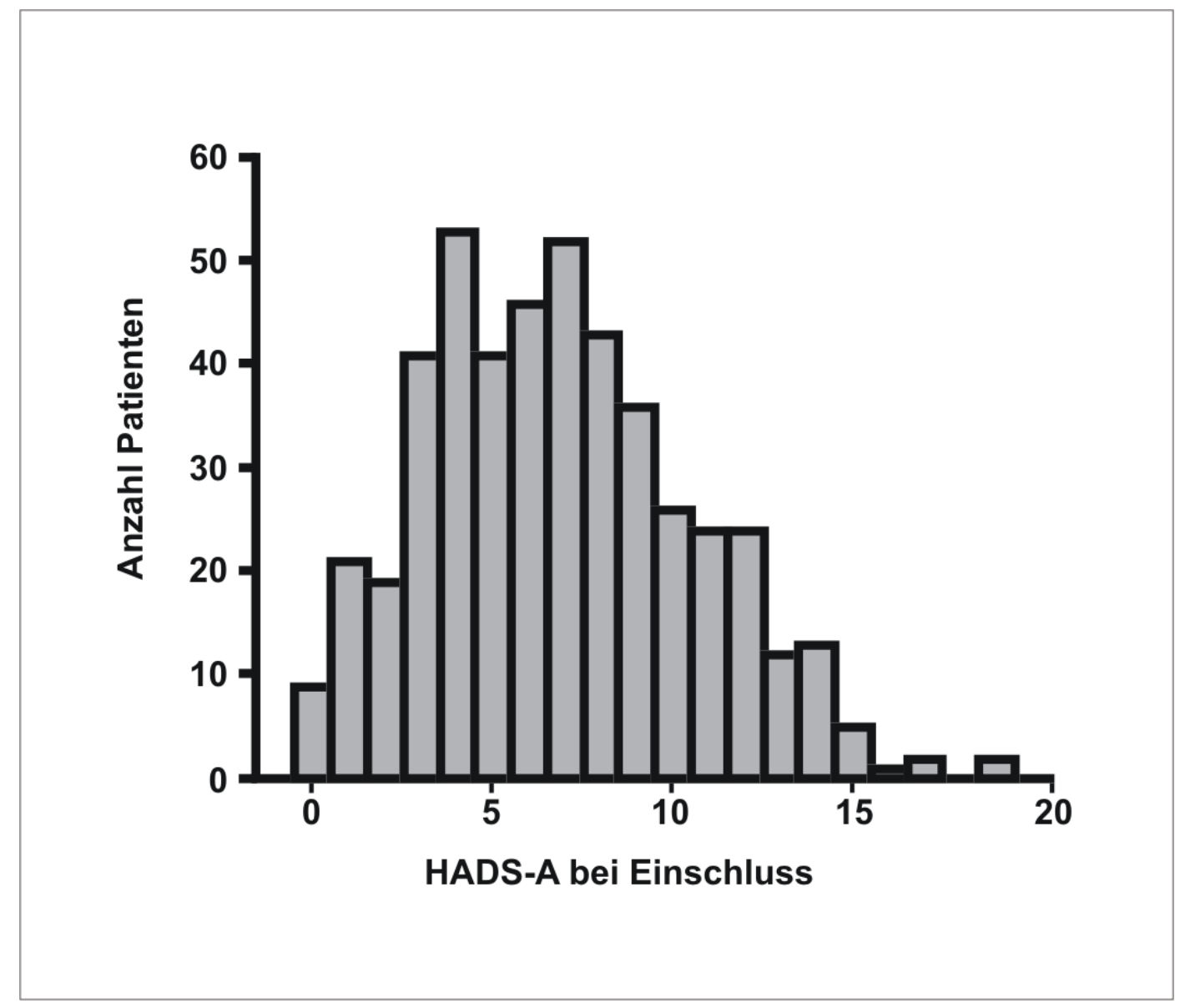

Abb. 14: Nicht normal verteilte Hospital Anxiety and Depression Scale-Werte für Angst bei Einschluss

\subsubsection{Gruppenvergleich von ängstlichen und nicht-ängstlichen Patienten}

Um den Vergleich der drei unteren Angstquartile mit dem obersten Quartil herzustellen wurden die Basisdaten, die zu Beginn der Studie aufgenommen wurden, auf signifikante Unterschiede untersucht. Bei den 356 Studienteilnehmern aus den unteren drei Angstquartilen (HADS-A<10) ergab sich ein gemittelter Gesamtwert von 5,3 (SD 2,4). Bei den 106 Patienten aus dem obersten Angstquartil (HADS-A $\geq 10$ ) zeigte sich ein Durchschnittswert von 12,1 (SD 1,9). Die beiden Subgruppen unterschieden sich signifikant $(\mathrm{p}<0,001)$ in Bezug auf das Geschlecht, wobei in den unteren drei Angstquartilen mehr Männer (80,6\%) enthalten waren, verglichen mit dem vierten Quartil (63,2\%). Die ängstlicheren Patienten waren mit einem Durchschnittsalter von 59,2 (SD 9,1) Jahren signifikant jünger als die weniger ängstlichen Patienten mit einem gemittelten Alter von 65,1 (SD 9,7) Jahren ( $<<0,001)$. Eine Stenose von über 70\% in mehr als einem Koronargefäß zeigte sich vornehmlich in der weniger ängstlichen 
Subgruppe (54,5\%) verglichen mit der ängstlicheren Gruppe $(42,5 \%), p=0,035$. Alle anderen Basisdaten, die bei Einschluss erhoben wurden, unterschieden sich nicht signifikant zwischen den beiden Subgruppen. Die ausführlichen Ergebnisse dieses Gruppenvergleichs finden sich im Anhang in der Tabelle C.5.

\subsubsection{Testung der 3. Hypothese: Angst ist mit verbessertem Überleben assoziiert}

Um den Einfluss der Angst auf die Gesamtmortalität zu bestimmen, wurde das oberste Angstquartil (2 Todesfälle $=1,9 \%)$ mit dem ersten (13 Todesfälle=9,2\%, p=0,018), dem zweiten $(15$ Todesfälle $=17,2 \%, \mathrm{p}<0,001)$ und dem dritten Quartil $(14$ Todesfälle $=11,0 \%, \mathrm{p}=0,006)$ verglichen. Dabei zeigten sich zwischen allen Quartilen global signifikante Differenzen $(p=0,004)$. Bei einem Vergleich untereinander wiesen die Quartile eins bis drei keine signifikanten Unterschiede $(\mathrm{p}=0,173)$ auf. Bei einem Vergleich der zusammengefassten unteren drei Quartile $(11,8 \%)$ mit dem obersten Angstquartil (1,9\%) zeigte sich ebenfalls ein signifikanter Wert $(\mathrm{p}=0,002)$. Abbildung 15 zeigt den Vergleich der Angstquartilen in Bezug auf die Mortalitätsrate.

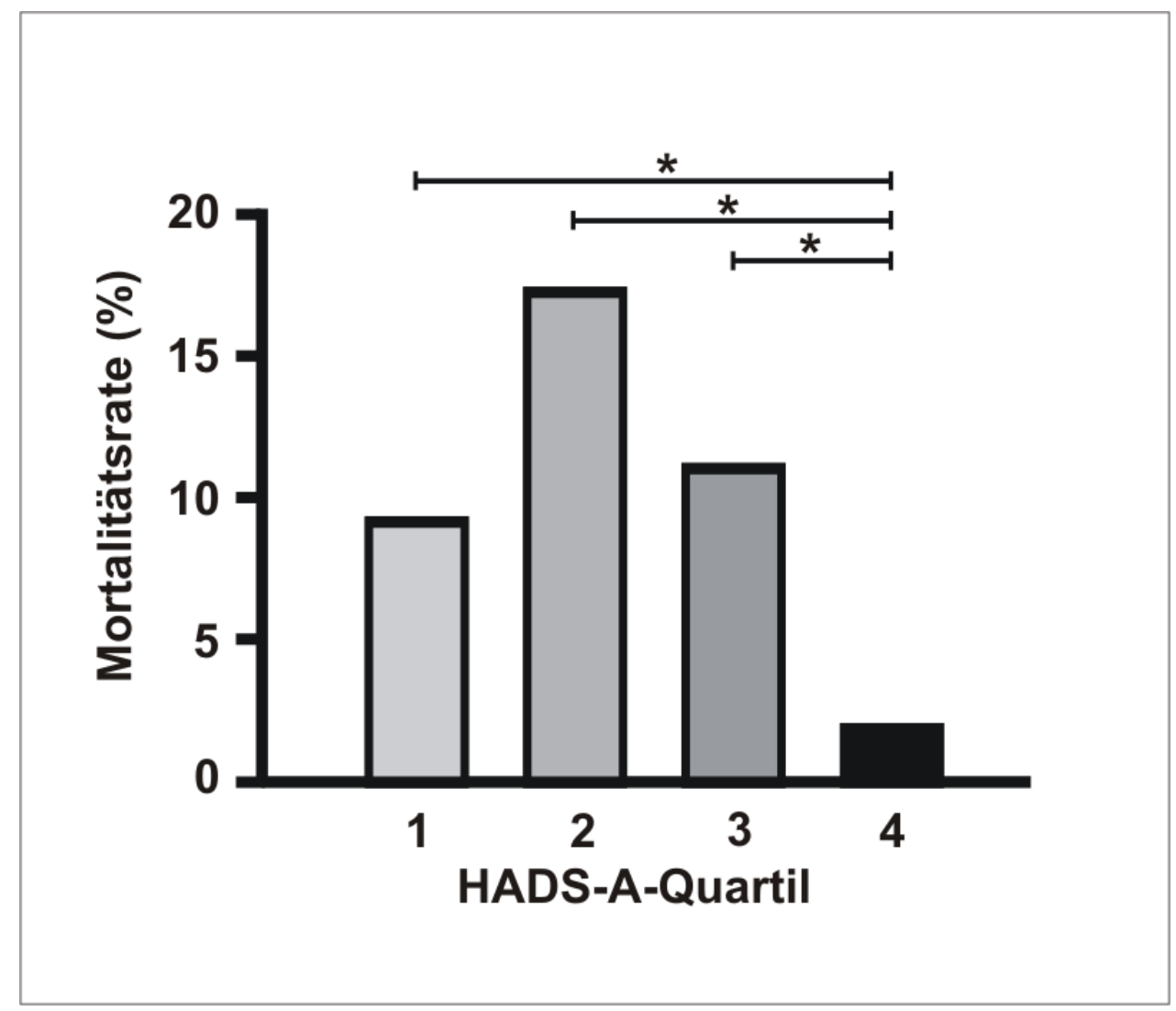

Abb. 15: Angst-Quartilenvergleich bezogen auf die Mortalitätsrate. Es zeigten sich signifikant $(p<0,018)$ weniger Todesfälle im vierten ängstlicheren Quartil, verglichen mit allen anderen Quartilen. Signifikante Unterschiede sind mit Balken und Sternchen gekennzeichnet. 
Bei der Betrachtung der unadjustierten Kaplan-Meier-Darstellung (vgl. Abb. 16) bestätigt sich der protektive Einfluss der Angst in Bezug auf die Gesamtmortalität ( $\mathrm{p}=0,003)$.

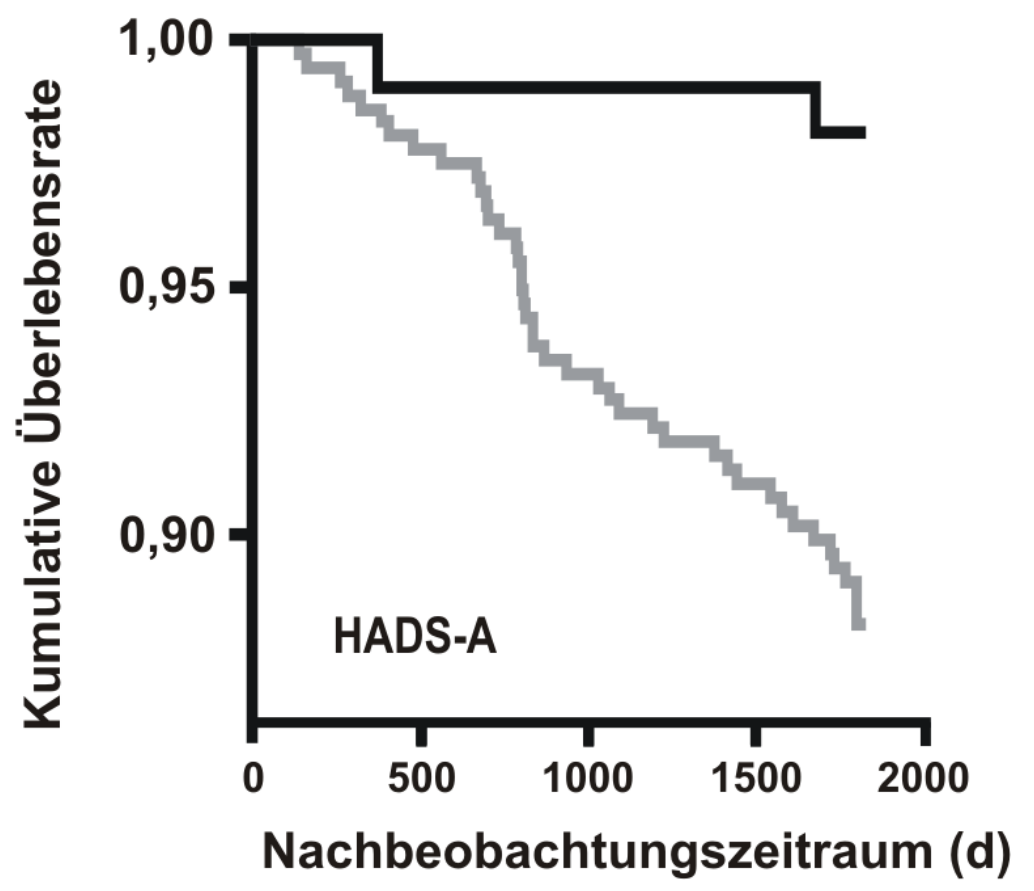

Abb. 16: Kumulative Überlebensrate nach PCI. Dargestellt ist das kumulative Überleben nach initialer Stentimplantation über 5 Jahre Nachbeobachtungszeitraum. Es zeigten sich signifikant $(p=0,003)$ weniger Todesfälle bei den ängstlichen Patienten (schwarze Linie), verglichen mit weniger ängstlichen Patienten (graue Linie).

\subsubsection{Einflussnahme somatischer Basisdaten auf den Zusammenhang der Gesamtmortalität} mit der Angst

Als nächstes sollte der Fragestellung nachgegangen werden, ob neben der Angst noch andere Basisdaten mit der Gesamtmortalität assoziiert waren und ob der Angsteffekt hiervon unabhängig bestehen blieb bzw. ob sich dieser durch die Hereinnahme verändert. Zu diesem Zweck wurde im Cox-Regressionsmodell nach Adjustierung aller relevanten, potenziellen Confounder (vgl. 3.5.7) auf Signifikanz getestet, wobei sich für das Gesamtmodell ein pWert $<0,001$ ergab. 
Es zeigte sich für die Angst ein unabhängiger Effekt, der mit einer niedrigeren Mortalitätsrate einher ging $(\mathrm{HR}=0,21,95 \% \mathrm{CI}=0,05-0,91, \mathrm{p}=0,037)$. Der Body-Mass-Index war ebenfalls negativ mit dem Überleben assoziiert $(\mathrm{HR}=0,90,95 \% \mathrm{CI}=0,82-0,99, \mathrm{p}=0,038)$. Confounder mit einer assoziierten höheren Mortalitätsrate waren Alter bei Einschluss (HR=1,04, 95\% CI=1,01,08, $\mathrm{p}=0,035)$, Tabakabusus bei Einschluss ( $\mathrm{HR}=2,71,95 \% \mathrm{CI}=1,29-5,71, \mathrm{p}=0,009)$ und $\mathrm{Zu}$ stand nach Myokardinfarkt bei Einschluss ( $\mathrm{HR}=1,91,95 \% \mathrm{CI}=1,0-3,63, \mathrm{p}=0,048)$. Alle anderen Variablen aus dem Basisdatensatz waren nicht signifikant.

Unabhängig von anderen Basisdaten zeigte sich für Patienten mit Angst im obersten Quartil ein signifikant erniedrigtes Mortalitätsrisiko.

Die Ergebnisse dieser Cox-Regressionsanalyse finden sich im Anhang in der Tabelle C.1.

\subsubsection{Protektiver Einfluss der Angst auf die Major Adverse Cardiovascular Events}

Das Auftreten der Major Adverse Cardiovascular Events (vgl. Tabelle 13) sollte auf einen Zusammenhang zum psychischen Parameter Angst überprüft werden. Ein Vergleich des vierten Angstquartils (7 MACEs=6,5\%) mit dem ersten Angstquartil (25 MACEs=17,6\%, $\mathrm{p}=0,01)$, dem zweiten (18 MACEs=20,7\%, p=0,003) und dem dritten (23 MACEs=18,0\%, $\mathrm{p}=0,009)$ zeigten signifikante Unterschiede. Ebenfalls zeigten sich zwischen allen Quartilen global signifikante Differenzen $(\mathrm{p}=0,026)$.

Tabelle 13: Verteilung der Major Adverse Cardiovascular Events auf die Angstquartile

\begin{tabular}{|c|c|c|c|}
\hline Angstquartil & Tod & $\begin{array}{c}\text { Akuter, nicht fata- } \\
\text { ler Myokardinfarkt }\end{array}$ & Apoplex \\
\hline $\mathbf{1}$ & 13 & 9 & 4 \\
\hline $\mathbf{2}$ & 15 & 3 & 8 \\
\hline $\mathbf{3}$ & 14 & 3 & 0 \\
\hline $\mathbf{4}$ & 2 & 5 & 13 \\
\hline Gesamt & 44 & 20 & 13 \\
\hline
\end{tabular}

Ein Vergleich der drei untersten Quartile untereinander führte zu keinem signifikanten Ergebnis $(p=0,34)$. Die Patienten aus dem obersten Angstquartil $(6,6 \%)$ waren weniger von MACEs 
betroffen als die nicht-ängstlichen Patienten $(18,5 \%, \mathrm{p}=0,003)$ aus den zusammengefassten unteren drei Quartilen. Die Abbildung 17 zeigt den Quartilenvergleich in Bezug auf die Major Adverse Cardiovascular Events.

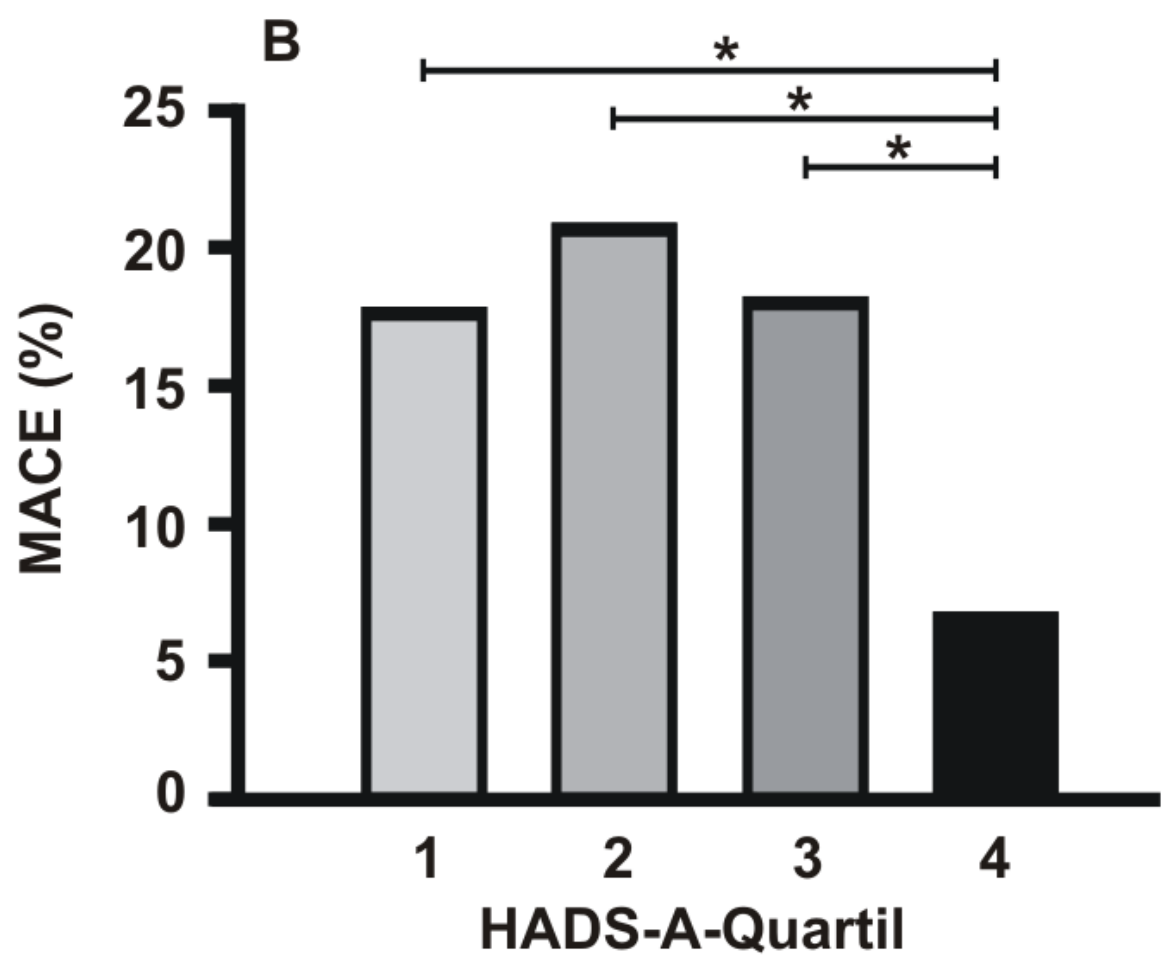

Abb. 17: Angst-Quartilenvergleich bezogen auf die Major Adverse Cardiovascular Events. Es zeigten sich signifikant $(p \leq 0,01)$ niedrigere Ereignisraten im vierten ängstlicheren Quartil, verglichen mit jedem der anderen Quartile. Signifikante Unterschiede sind mit Balken und Sternchen gekennzeichnet.

Bei der Betrachtung der unadjustierten Kaplan-Meier-Darstellung (vgl. Abb. 18) bestätigt sich der protektive Einfluss der Angst in Bezug auf das kumulative ereignisfreie Überleben $(\mathrm{p}=0,003)$. Interessanterweise setzt der protektive Effekt der Angst erst nach einer gewissen Zeit ein (ca. nach 700 Tagen). 


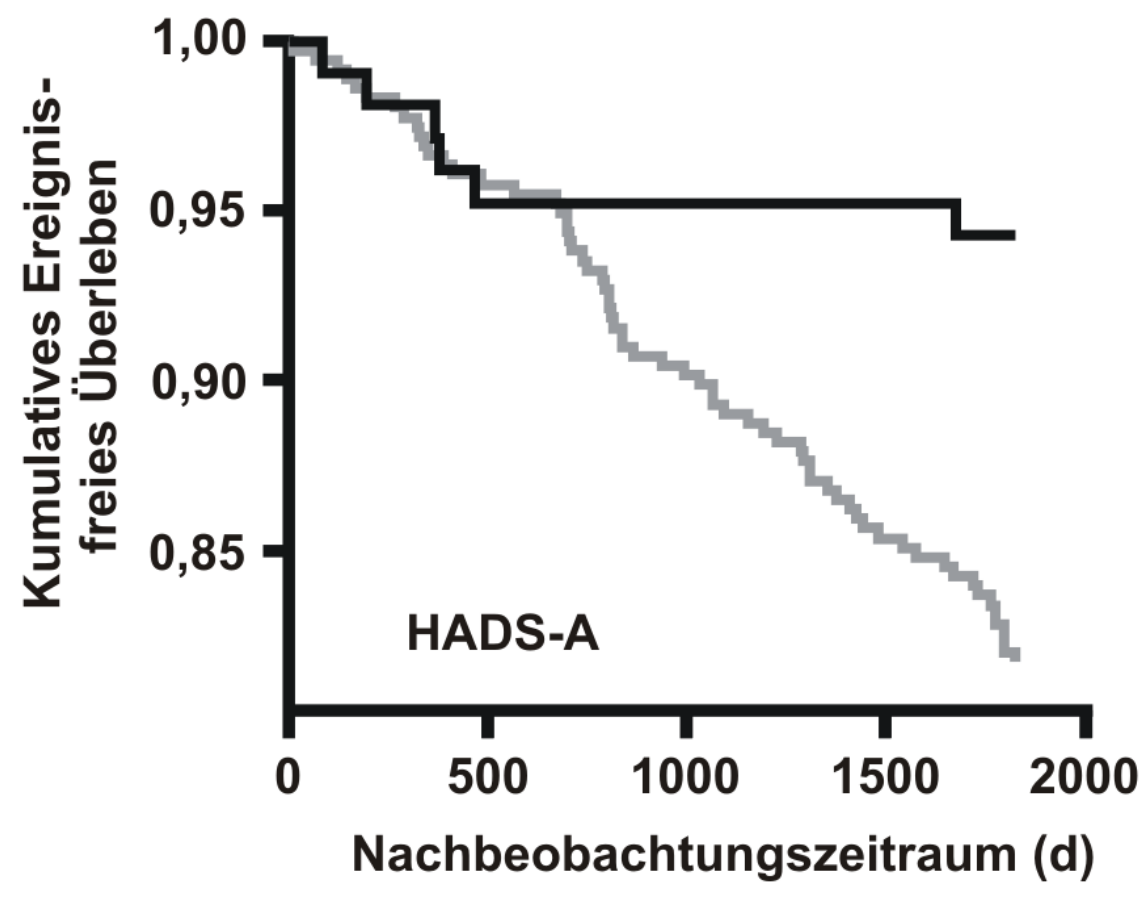

Abb. 18: Kumulatives ereignisfreies Überleben nach PCI. Dargestellt ist das kumulative ereignisfreie Überleben nach initialer Stentimplantation über 5 Jahre Nachbeobachtung. Es zeigten sich signifikant ( $\mathrm{p}=0,003)$ weniger Major Adverse Cardiovascular Events bei den ängstlichen Patienten (schwarze Linie), verglichen mit weniger ängstlichen Patienten (graue Linie).

4.4.5 Einflussnahme somatischer Basisdaten auf den Zusammenhang der MACEs mit der Angst

Es sollte überprüft werden, ob neben der Angst noch andere Basisdaten mit der Rate der Major Adverse Cardiovascular Events assoziiert waren und ob der Angsteffekt hiervon unabhängig bestehen blieb. Diese Fragestellung wurde erneut in einem Cox-Regressionsmodell nach Adjustierung aller relevanten, potenziellen Confounder, die dem Basisdatensatz entnommen wurden, überprüft. Für das Gesamtmodell ergab sich ein p-Wert<0,001.

Es zeigte sich für die Angst ein unabhängiger Effekt, der mit einer erniedrigten Rate an MACEs einher ging ( $\mathrm{HR}=0,34,95 \% \mathrm{CI}=0,14-0,80, \mathrm{p}=0,014)$. In gleicher Richtung zeigte sich der Effekt bei der proximalen Stentlokalisation (HR=0,56, 95\%CI=0,32-0,98, $\mathrm{p}=0,042)$. Confounder mit einer assoziierten höheren Rate an MACEs waren Tabakabusus bei Einschluss $(\mathrm{HR}=2,82,95 \% \mathrm{CI}=1,58-5,03, \mathrm{p}<0,001)$, Zustand nach Myokardinfarkt bei Einschluss 
( $\mathrm{HR}=1,76,95 \% \mathrm{CI}=1,06-2,92, \mathrm{p}=0,029)$ und Zustand nach Bypass-Operation $(\mathrm{HR}=2,13$, 95\% CI $=1,06-4,28, \mathrm{p}=0,034)$. Alle anderen Variablen aus dem Basisdatensatz waren nicht signifikant.

Unabhängig von anderen Basisdaten zeigte sich für Patienten mit Angst im obersten Quartil eine signifikant erniedrigte Rate an Major Adverse Cardiovascular Events.

Die Ergebnisse dieser Cox-Regressionsanalyse finden sich im Anhang in der Tabelle C.2.

\subsubsection{Erhöhte Revaskularisationsraten bei Angst im obersten Quartil}

Anders als bei der bisher gezeigten Gesamtmortalität und bei dem kumulativen ereignisfreien Überleben zeigte sich ein umgekehrter Effekt auf die Wahrscheinlichkeit einer Revaskularisation innerhalb der fünfjährigen Nachbeobachtung. Die höchste Revaskularisationsrate mit insgesamt 28 Maßnahmen (25,7\%) wurde in der Gruppe der KHK-Patienten mit klinisch relevanter Angst beobachtet. Ein signifikanter Unterschied zeigte sich zwischen dieser Gruppe und den Patienten aus dem dritten Quartil, in der nur 19 revaskularisierende Interventionen $(14,5 \%)$ durchgeführt wurden $(\mathrm{p}=0,03)$. Verglichen mit dem vierten Quartil wurden bei den Studienteilnehmern im ersten HADS-A-Quartil 27 Maßnahmen $(18,9 \%, p=0,195)$ und im zweiten Quartil 13 Maßnahmen (14,9\%, p=0,066) durchgeführt. Der Quartilenvergleich mittels Chi-Quadrat-Test über alle vier Gruppen ergab einen p-Wert von 0,118.

Tabelle 14: Verteilung der Revaskularisationsmaßnahmen auf die Angstquartile

\begin{tabular}{|c|c|c|c|}
\hline Angstquartil & $\begin{array}{c}\text { Erneute PCI mit } \\
\text { Stentimplantation }\end{array}$ & PCI & $\begin{array}{c}\text { Koronararterien- } \\
\text { Bypass-Operation }\end{array}$ \\
\hline $\mathbf{1}$ & 19 & 3 & 7 \\
\hline $\mathbf{2}$ & 12 & 2 & 4 \\
\hline $\mathbf{3}$ & 15 & 2 & 5 \\
\hline $\mathbf{4}$ & 26 & 9 & 16 \\
\hline Gesamt & 72 & 2 & 0 \\
\hline
\end{tabular}


Ein Vergleich der drei untersten Quartile untereinander zeigte kein signifikantes Ereignis $(\mathrm{p}=0,570)$. Die Rate war, verglichen mit den weniger ängstlichen Studienteilnehmern aus den zusammengefassten unteren drei Quartilen bei den ängstlichen Patienten erhöht (26,4\% versus 16,6\%), wobei der p-Wert bei 0,028 lag (vgl. Abb. 19).

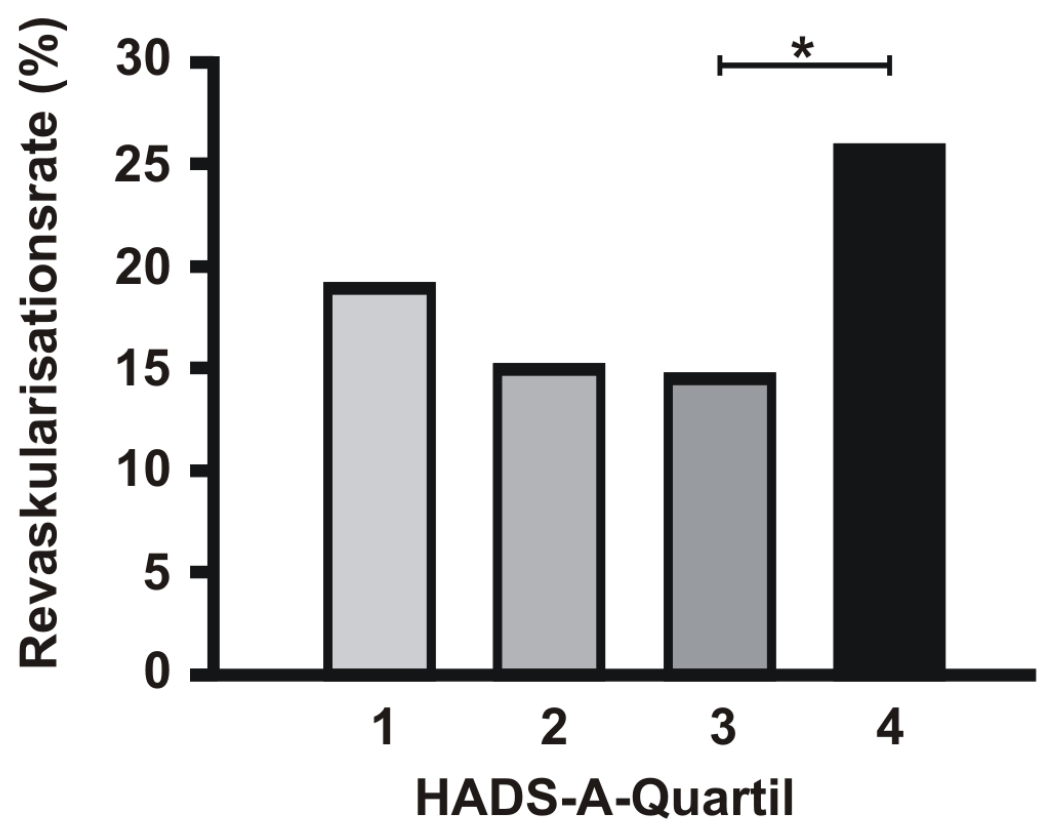

Abb. 19: Angst-Quartilenvergleich bezogen auf die Revaskularisationsrate. Es zeigte sich eine signifikant (p=0,028) höhere Revaskularisationsrate im vierten Quartil verglichen mit dem dritten Quartil. Der univariate Unterschied zwischen den Gruppen ist mit Balken und Sternchen gekennzeichnet.

Bei der Betrachtung der unadjustierten Kaplan-Meier-Darstellung (vgl. Abb. 20) bestätigt sich das erhöhte Risiko der Revaskularisation beim Vorliegen von erhöhter Angst ( $p=0,02)$. 


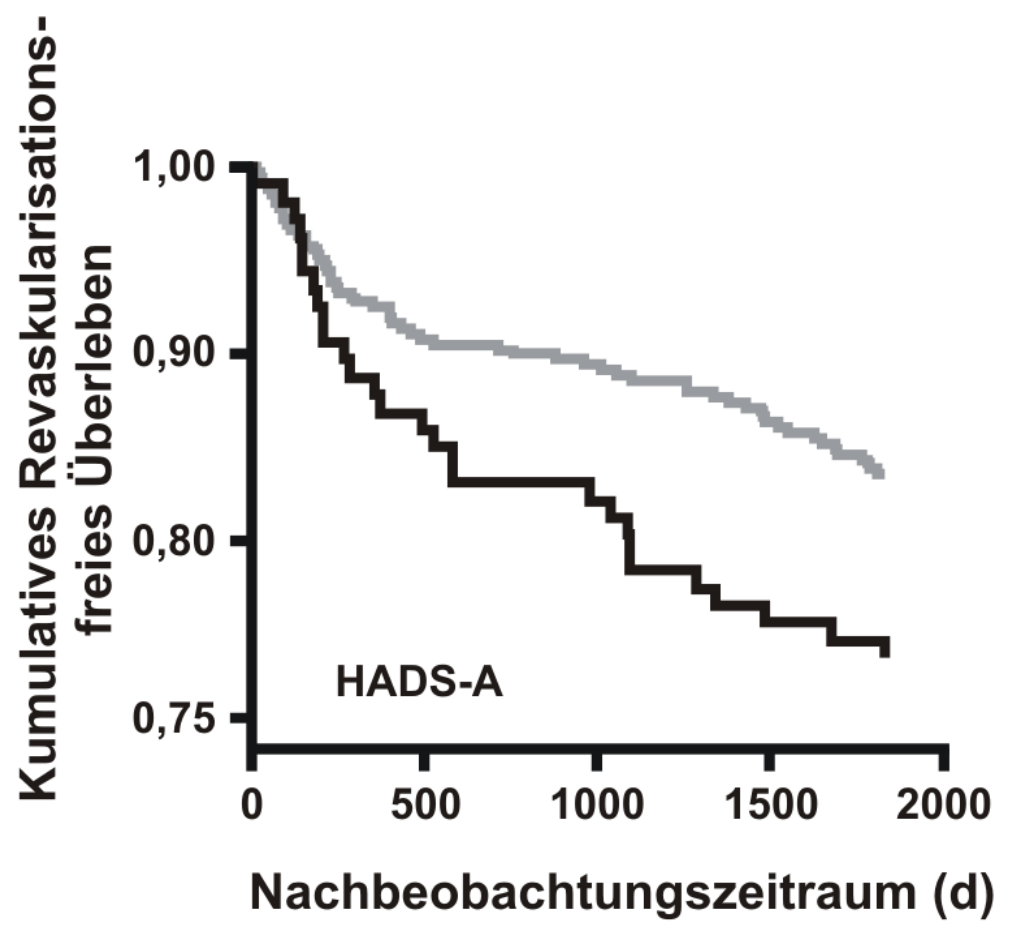

Abb. 20: Revaskularisationsrate nach PCI. Dargestellt ist das kumulative revaskularisationsfreie Intervall nach initialer Stentimplantation über 5 Jahre Nachbeobachtung. Es ergaben sich signifikant $(p=0,02)$ mehr Fälle bei ängstlichen Patienten (schwarze Linie), verglichen mit weniger ängstlichen Patienten (graue Linie).

4.4.7 Einflussnahme somatischer Basisdaten auf den Zusammenhang der Revaskularisationsrate mit der Angst

Im Folgenden soll multivariat analysiert werden, ob die Assoziation der Angst mit der Revaskularisationsrate unabhängig von den Basisdaten bestehen blieb. Da bei einer Auswahl der gleichen Confounder wie bisher das Gesamtmodell insignifikant war, besteht für die Angst im volladjustierten Modell kein unabhängiger Wert.

Um Überadjustierung zu vermeiden wurden in einer zweiten Berechnung nur diejenigen Kovariablen ausgewählt, für die sich in der univariaten Analyse ein signifikanter Unterschied zwischen ängstlichen und nicht-ängstlichen Patienten ergab. Das Cox-Regressionsmodell soll nach Adjustierung dieser beiden übrig gebliebenen Variablen „Angstwert liegt im obersten Quartil“ und „Zustand nach Bypass-Operation bei Einschluss“ darüber Auskunft geben. Für das Gesamtmodell ergab sich ein signifikanter p-Wert von 0,003 . 
Für die ängstlichen Patienten zeigte sich ein klarer Trend eines erhöhten Risikos einer erneuten Revaskularisation $(\mathrm{HR}=1,62,95 \% \mathrm{CI}=1,0-2,63, \mathrm{p}=0,051)$. Die für dieses Modell ausgewählte Einflussgröße „Zustand nach Bypass-Operation bei Einschluss“ zeigte sich signifikant (HR=2,26, 95\%CI=1,25-4,1, p=0,007). Die Ergebnisse dieser beiden Cox-Regressionsanalysen finden sich im Anhang in der Tabelle C.3 und C.4.

\subsubsection{Annahme der 3. Hypothese}

Es ist analysiert worden, ob die Angst, unabhängig von anderen somatischen Parametern, einen signifikanten Einfluss auf die Prognose der Patienten nach PCI hatte. Für die beschriebenen Endpunkte zeigte sich für diesen psychologischen Parameter für den Nachbeobachtungszeitraum von 5 Jahren ein signifikanter Zusammenhang. In Bezug auf die Gesamtmortalität und auf die Major Adverse Cardiovascular Events hatte erhöhte Angst einen inversen $\mathrm{Zu}$ sammenhang mit der Ereignisrate. Dies bestätigte sich in den multivariaten Analysen und könnte auf einen protektiven Effekt der Angst hindeuten. Umgekehrt erfolgten signifikant mehr Revaskularisationsmaßnahmen bei dieser Subgruppe. Dieser Effekt war jedoch im volladjustierten Modell nicht, im teiladjustierten Modell nur marginal signifikant und somit möglicherweise artifiziell. Da die Angst als Prädiktionsfaktor innerhalb der 5 Jahre für zwei der drei Endpunkte eminent war und bivariat auch für die Revaskularisationsrate bestand, kann die dritte Hypothese überwiegend angenommen werden. 


\section{DISKUSSION}

\subsection{Studienziel und Resultat}

Die vorliegende Längsschnittstudie hatte das Ziel, den Zusammenhang zwischen psychischen Faktoren und deren Einfluss auf die Prognose bzw. auf das weitere Krankheitsgeschehen von 470 KHK-Patienten über einen Beobachtungszeitraum von 5 Jahren nach PCI zu beobachten und zu analysieren. Dabei wurden die Parameter Angst, Depressivität und das Vorliegen einer Typ-D-Persönlichkeit bei Einschluss betrachtet und mit den Endpunkten Mortalität, Major Adverse Cardiovascular Events und Revaskularisation in Verbindung gebracht.

Es zeigte sich ein protektiver Einfluss der Angst sowohl auf das Überleben als auch auf die Major Adverse Cardiovascular Events, vergleichbar mit den Studien von Mykletun et al. (2007) und Meyer et al. (2010). Andere Autoren wiesen dagegen ein erhöhtes Mortalitäts- und Myokardinfarktsrisiko für ängstliche Herzpatienten nach (Haines et al. 1987; Kawachi et al. 1994a; Kawachi et al. 1994b; Frasure-Smith et al. 1995b; Moser und Dracup 1996; Albert et al. 2005; Rothenbacher et al. 2007; Moser et al. 2007; Shibeshi et al. 2007; Frasure-Smith et al. 2008; Shen et al. 2008; Denollet et al. 2009b; Roest et al. 2010a; Moser et al. 2011). Für die Depressivität ergab sich ein prädiktiver Wert für die Gesamtmortalität bei Betrachtung des 2-jährigen Nachbeobachtungsintervalls, was mit der Literatur übereinstimmt (Barth et al. 2004, Nicholson et al. 2006), jedoch nicht für den vollständigen Nachbeobachtungszeitraum von 5 Jahren. Andere Autoren beschreiben auch für diesen längeren Zeitraum eine erhöhte Ereignisrate bei Patienten mit Depressivität (Lesperance et al. 2002; Grace et al. 2005).

Das Vorliegen einer Typ-D-Persönlichkeit hatte in dieser Kohorte - wie auch in mehreren anderen Studien - keine prognostische Relevanz (Pelle et al. 2010; Coyne et al. 2011; Grande et al. 2011; Volz et al. 2011; Damen et al. 2013). Gleichzeitig unterscheiden sich die in dieser Studie gezeigten Ergebnisse von anderen prognostischen Untersuchungen der Arbeitsgruppe um Denollet, die oftmals einen unabhängigen signifikanten Prädiktionswert der Typ-DPersönlichkeit gezeigt hatten (Denollet et al. 1996; Denollet und Brutsaert 1998; Denollet et al. 2000; Pedersen et al. 2004; Denollet et al. 2006a; Pedersen et al. 2007b; Martens et al. 2010).

Um die vorliegenden Ergebnisse in den aufgeführten aktuellen Literaturkontext einzuordnen, ist es unumgänglich, die Vergleichbarkeit dieser klinisch gut charakterisierten Stichprobe mit 
vorangegangenen Arbeiten zu überprüfen und Gründe für die Divergenz der Resultate zu ermitteln. Die Patientenkohorte und das Studiendesign sollen im Folgenden diskutiert werden.

\subsection{Studiendesign und Begrenzungen der Studie}

Patienten wurden zum Zeitpunkt der Implantation eines De-novo-Koronarstents in die Studie eingeschlossen. Vorteil dieser Herangehensweise ist die Selektion einer bestimmten und gut abgegrenzten Patientenklientel, da nicht - wie in anderen Studien (Herrmann et al. 1998; Grande et al. 2011; Nakamura et al. 2013) - gemischte Herzpatientenkohorten untersucht wurden, sondern sich die Ergebnisse auf die koronare Herzkrankheit und eine relativ homogene Ausgangssituation beschränkten. So können die Ergebnisse dieser Studie mit anderen Resultaten von Follow-Up-Studien nach PCI gut verglichen werden. Eine Einschränkung der Studie war jedoch, dass nicht einheitlich jeder Patient nach Platzieren des ersten Koronarstents nachbeobachtet wurde, sondern viele Patienten bereits in einem anderen Koronargefäß einen Stent (oder mehrere) implantiert bekommen hatten. So bestand die untersuchte Patientenkohorte aus Studienteilnehmern, die zum einen Teil bereits mehrfach gestentet wurden und bei denen die KHK unter Umständen bereits fortgeschrittenen war und zum anderen Teil aus Patienten mit nur einem Koronarstent und entsprechend leichter KHK bzw. kurzem Verlauf vor der Index-PCI. Ein ähnliches Studiendesign war ebenfalls Grundlage anderer Studien, die die PCI als Einschlusskriterium definierten (Pedersen et al. 2009; Damen et al. 2013).

Eine weitere Begrenzung dieser Studie ist, dass die psychischen Parameter Angst, Depressivität und Typ-D-Persönlichkeit bei Einschluss nur durch ein psychometrisches Screening via Selbsteinschätzung gemessen und nicht mittels eines strukturierten klinischen Patienteninterviews erfasst wurden. Es lässt sich also nicht schlussfolgern, ob die vorliegenden Ergebnisse auf spezifische Angststörungen übertragbar sind oder vorrangig für unspezifisch erhöhte Ängstlichkeit gültig sind. Dennoch hat sich der in dieser Studie verwendete HADSFragebogen in der Vergangenheit als besonders geeignet erwiesen, um Angst bei somatisch erkrankten Menschen (darunter auch KHK-Patienten) zu ermitteln (Herrmann 1997; Spinhoven et al. 1997; Bjelland et al. 2002; Rothenbacher et al. 2007). Für die Identifikation des Typ-D ist ein strukturiertes Interview nicht etabliert, die DS14 stellt hier das auch in der Literatur verwandte Standardverfahren dar.

Eine weitere Limitation dieser Studie liegt in der Tatsache begründet, dass die psychometrische Messung im Rahmen der Implantation des Koronarstents erfolgte. Im Zuge der Intervention erhielten die Patienten, während sie im Klinikum Links der Weser waren, die Fragebö- 
gen. Die Erfassung der Angst zu diesem frühen Zeitpunkt, anders als einen Monat nach PCI, könnte von situationsbezogenen Faktoren beeinflusst worden sein (Poston et al. 2003).

Da es in Deutschland kein Zentralregister gibt, konnte die genaue Todesursache nur für einige der verstorbenen Patienten festgestellt werden. Die verfügbaren Daten waren unzureichend, um die kardiale Todesrate verlässlich festzustellen, weswegen alle verstorbenen Patienten, unabhängig von der Todesursache, in einem Endpunkt (all-cause mortality: Gesamtmortalität) erfasst wurden.

Außerdem wurden die Daten nur aufgrund von Beobachtungen gewonnen, weshalb kausale Schlussfolgerungen inadäquat sein könnten. Eine Erklärung des Outcomes der Studienteilnehmer aufgrund von z.B. veränderten physiologischen Parametern (Veränderung des Kortisolspiegels, Zytokinausschüttung, Sympatikusaktivierung usw.) kann diese Studie nicht liefern. Des Weiteren fehlen Angaben zum Verhalten der Patienten im Nachbeobachtungszeitraum. Abgesehen vom Tabakabusus bei Einschluss gibt es keine Angaben zum gesundheitsbezogenen Verhalten des Patienten wie z.B. Frequenz der Arztbesuche, Essgewohnheiten oder Sport. Auch Angaben zur Teilnahme der Studienteilnehmer an kardialen Rehabilitationsmaßnahmen oder Einnahme von Psychopharmaka im weiteren Verlauf konnten nicht als potenziell relevante Confounder in den multivariablen Analysen verwendet werden. Diese Aspekte könnten zu einem Abweichen von den in der Literatur zu findenden Ergebnissen beigetragen haben.

\subsection{Repräsentativität der Kohorte}

Die beschriebene Patientenkohorte soll nachfolgend anhand der soziodemographischen Angaben und anhand des verwendeten Studiendesigns mit anderen Arbeiten verglichen werden. In Betracht kommen dabei die oben zitierten Studien.

\subsubsection{Soziodemographische Angaben}

Bei der beschriebenen Analyse des Gesamtkollektivs zeigte sich ein geringer Frauenanteil von nur 23,2\%. In anderen Follow-Up-Studien ergaben sich ähnliche Geschlechteraufteilungen, was wohl daran liegt, dass es sich um eine kardiologische Patientenkohorte handelt und das männliche Geschlecht als bekannter Risikofaktor für eine frühere Manifestation der KHK etabliert ist (Müller-Nordhorn und Willich 2008). Auch das Durchschnittsalter von 63,7 $(9,9)$ 
liegt nur geringfügig über dem Mittelwert anderer psychokardiologischer Untersuchungen (vgl. Tabelle 15).

Tabelle 15: Anteil an weiblichen Patienten und Durchschnittsalter in anderen Studien

\begin{tabular}{|l|c|c|c|}
\hline \multicolumn{1}{|c|}{ Studie } & Frauenanteil (\%) & Durchschnittsalter & Kohorte \\
\hline $\begin{array}{l}\text { Frasure - Smith und } \\
\text { Lesperance 2008 }\end{array}$ & 19,3 & $60(10,6)$ & 804 KHK-Patienten \\
\hline Sullivan et al. 2003 & 17,2 & $62,0(9,3)$ & 198 PCI-Patienten \\
\hline Damen et al. 2013 & 28 & $62,0(11,1)$ & 1234 PCI-Patienten \\
\hline Moser et al. 2007 & 34 & $62(14)$ & 536 MI-Patienten \\
\hline Schmidt et al. 2011 & 37 & $60,2(10,1)$ & 137 PCI-Patienten \\
\hline Pedersen et al. 2009 & 27,9 & $62,5(11,5)$ & 574 PCI-Patienten \\
\hline Grande et al. 2011 & 22,5 & $63,3(10,7)$ & 977 gemischte Kohorte \\
\hline Denollet et al. 2005 & 28 & $62,2(10,9)$ & 875 PCI-Patienten \\
\hline
\end{tabular}

In den sehr entscheidenden Basisparametern Alter, Geschlecht und Einschlusskriterium bestehen keine relevanten Differenzen zwischen der hier dargestellten Studienkohorte und denjenigen aus Tabelle 15. Die Anzahl von 470 Patienten fällt etwas geringer aus als der Durchschnitt der genannten Studien, jedoch ist die Nachbeobachtungsrate mit einer Follow-UpQuote von $98,3 \%$ sehr hoch.

\subsubsection{Nachbeobachtungszeitraum und Endpunkte}

Der in dieser Studie gewählte Nachbeobachtungszeitraum von 5 Jahren ist vergleichbar mit ähnlichen prospektiven Studien (Sullivan et al. 2003; Grande et al. 2011). Das Zeitintervall war lang genug, um die Auswirkungen unterschiedlicher psychischer Faktoren zu erfassen und eignete sich somit für eine Risikoeinschätzung der untersuchten Kohorte.

Die Einteilungen und Klassifizierungen der in dieser Studie verwendeten Endpunkte sind allerdings nur teilweise mit denjenigen anderer Untersuchungen vergleichbar. Die größte Übereinstimmung findet sich im Endpunkt Mortalität, weil andere Kohorten ebenfalls unter diesem isolierten Gesichtspunkt analysiert wurden. 
Die Definition der Major Adverse Cardiovascular Events ist bei Betrachtung der aktuellen Literatur jedoch nicht einheitlich. Dieser composite endpoint (engl.: zusammengesetzter Endpunkt) variiert von Studie zu Studie, was die Vergleichbarkeit erschwert. In dieser Studie wurden die MACEs als schwerwiegende klinische Ereignisse verstanden (Tod, nicht-fataler Myokardinfarkt, Apoplex) und sollten nicht mit Endpunkten der Revaskularisation (PCI mit oder ohne Stentimplantation, Bypass-Operation) vermengt werden. Letztere stehen für medizinisch indizierte Maßnahmen, die nach der initialen Koronarstentintervention für notwendig erachtet wurden, um die Myokardperfusion zu verbessern. In diese Parameter gehen somit auch Parameter des Arzt- und indirekt des Patientenverhaltens ein. Die in dieser Studie gewählte Aufteilung hat demnach eine hohe klinische Relevanz, da hier klar zwischen klinischem Ereignis auf der einen Seite und medizinischer Intervention auf der anderen Seite unterschieden wurde.

Ähnliches gilt für die psychometrische Messung der untersuchten Parameter, da in der Vergangenheit unterschiedliche Fragebögen zur Erfassung der Angst und der Depressivität benutzt wurden bzw. in einigen Studien sogar ein klinisches Interview mit den Studienteilnehmern durchgeführt wurde (vgl. Tabelle 16). Selbst prospektive Studien, die den gleichen Fragebogen benutzten, sind gelegentlich nur bedingt miteinander vergleichbar, da häufig unterschiedliche Cut-Off-Werte verwendet wurden. Für den HADS-Fragebogen steht ein bestimmter Grenzwertbereich zur Feststellung von Angst und Depressivität zur Verfügung (z.B. für Angst zwischen $\geq 8$ und $\geq 11$ ). Je nach zu messendem Ausprägungsgrad eines psychologischen Merkmals entschieden sich die Autoren für unterschiedliche Werte, um zwischen ängstlichen und nicht-ängstlichen Patienten zu unterscheiden. Willkürlichkeit bei der Auswahl des in dieser Studie verwendeten Cut-Off-Wertes kann ausgeschlossen werden, da der Grenzwert in der vorliegenden Arbeit mithilfe der Quartilenaufteilung bestimmt wurde. So kamen die Grenzwerte von $\geq 10$ zur Bestimmung der Angst und von $\geq 8$ zur Bestimmung der Depressivität zustande. Diese gängigen Werte grenzen in dieser Kohorte das oberste Angst- bzw. Depressivitätsquartil von der restlichen Studienkohorte ab. Beispiele von vergleichbaren prospektiven Studien zeigt Tabelle 16. 
Tabelle 16: Psychometrische Messung, Nachbeobachtungsintervall und Endpunkte in anderen Studien

\begin{tabular}{|c|c|c|c|}
\hline Studie & $\begin{array}{c}\text { Psychometrische } \\
\text { Messung }\end{array}$ & $\begin{array}{c}\text { Zeitraum } \\
\text { (Jahre) }\end{array}$ & Endpunkte \\
\hline $\begin{array}{l}\text { Frasure - Smith und } \\
\text { Lesperance } 2008\end{array}$ & $\begin{array}{l}\text { HADS-A, Beck Depres- } \\
\text { sion Inventory II, DSM- } \\
\text { IV-basierte Diagnose }\end{array}$ & 2 & $\begin{array}{l}\text { MACE (kardialer Tod, Myo- } \\
\text { kardinfarkt, Herz-Kreislauf- } \\
\text { Stillstand, nicht-elektive Revas- } \\
\text { kularisation) }\end{array}$ \\
\hline Sullivan et al. 2003 & $\begin{array}{l}\text { DSM-IV-basierte Diag- } \\
\text { nose }\end{array}$ & 5 & $\begin{array}{l}\text { Bypass-OP, PCI, stationärer } \\
\text { Aufenthalt aus kardialen Grün- } \\
\text { den, Myokardinfarkt, Tod }\end{array}$ \\
\hline Damen et al. 2013 & HADS, DS14 & 7 & Tod \\
\hline Schmidt et al. 2011 & $\begin{array}{l}\text { DS14, Beck Depression } \\
\text { Inventory, Beck Anxiety } \\
\text { Inventory, Lipp Inventory } \\
\text { for Stress Symptoms for } \\
\text { Adults }\end{array}$ & 1 & $\begin{array}{l}\text { MACE (Tod, Myokardinfarkt, } \\
\text { PCI, Bypass-OP) }\end{array}$ \\
\hline Pedersen et al. 2009 & $\begin{array}{l}\text { Patient Health Question- } \\
\text { naire (PHQ-2) }\end{array}$ & 1,4 & MACE (Tod, Myokardinfarkt) \\
\hline Grande et al. 2011 & DS14, HADS & 5,9 & Tod \\
\hline Denollet et al. 2005 & HADS, DS14 & 0,75 & $\begin{array}{l}\text { MACE (Tod, Myokardinfarkt, } \\
\text { PCI, Bypass-OP) }\end{array}$ \\
\hline
\end{tabular}

\subsubsection{Prävalenz der Typ-D-Persönlichkeit}

In dieser Studienkohorte lag die Prävalenz der Typ-D-Persönlichkeit mit 144 (31\%) Patienten vergleichsweise hoch. In anderen Studien variierte der Anteil an Typ-D-positiven Patienten von $18 \%$ (Bergvik et al. 2010) bis 38,5\% (Williams et al. 2008), wobei Werte zwischen $20 \%$ und 30\% häufig sind. Es wurde jedoch argumentiert, dass die unterschiedliche Prävalenz der Typ-D-Persönlichkeit in Studienkohorten nicht entscheidend für das Outcome war (Grande et al. 2011) und auch andere Studien mit unterschiedlichen Prävalenzen von 20\% und 29\% konnten keinen Zusammenhang zwischen der Typ-D-Persönlichkeit und der Gesamtmortalität nachweisen (de Voogd et al. 2009; Pelle et al. 2010). 


\subsection{Diskussion der 1. Hypothese: Prognostische Effekte der Depressivität}

Es wurde untersucht, ob die mittels HADS-D erfasste Depressivität einen signifikanten Einfluss auf das weitere Krankheitsgeschehen und auf die Prognose von KHK-Patienten nach PCI hat. Die über 5 Jahre nach Einschluss erfassten Endpunkte Gesamtmortalität, Major Adverse Cardiovascular Events und Revaskularisation wurden dabei mit diesem psychischen Parameter in Zusammenhang gebracht. Für die Major Adverse Cardiovascular Events und für die Revaskularisation konnten keine signifikanten Ergebnisse gezeigt werden. Bei der Untersuchung der Gesamtmortalität zeigte sich für die 5-jährige Nachbeobachtung zwar ebenfalls keine Signifikanz, wohl aber eine schwache Tendenz. HADS-D-positive Patienten hatten deskriptiv ein leicht erhöhtes Risiko innerhalb des Follow-Up-Intervalls zu versterben (vgl. Abb. 4). Ein anderes Bild zeigte sich bei Betrachtung der 2-jährigen Gesamtmortalität, da hier die Rate bei Patienten mit Depressivität signifikant erhöht war. Dieser Zusammenhang zeigte sich unabhängig von zahlreichen nicht-psychischen Faktoren, u.a. von den etablierten kardiovaskulären Risikofaktoren. Für die anderen beiden Endpunkte konnte jedoch kein signifikanter Unterschied für einen 2-jährigen Nachbeobachtungszeitraum festgestellt werden.

Wie in der Einleitung ausführlich dargestellt wurde, sind in der Vergangenheit zahlreiche prospektive Studien erschienen, die ebenfalls die signifikante Beeinflussung der 2-jährigen Mortalitätsrate durch die Depressivität darstellen (Irvine et al. 1999; Carney et al. 2003; Kaptein et al. 2006; Denollet et al. 2007; Pedersen et al. 2007a; Frasure-Smith und Lesperance 2008). In einer KHK-Metaanalyse wurde die Verdoppelung des Mortalitätsrisikos für 2 Jahre anhand von 62 publizierten prospektiven Studien belegt, wobei sich dieser Effekt unabhängig von anderen Risikofaktoren zeigte (Barth et al. 2004).

Auch eine erhöhte Langzeitmortalitätsrate konnte in Studien belegt werden (Barefoot et al. 2000; Welin et al. 2000; Grace et al. 2005; Lesperance et al. 2002; Damen et al. 2013), was die Frage aufwirft, warum dieser Effekt in der hier vorliegenden Studienkohorte nicht nachzuvollziehen war, sondern die Depressivität wie in anderen Langzeitbeobachtungen (Herrmann et al. 2000; Stewart et al. 2003; Sullivan et al. 2003; Kornerup et al. 2011) keinen Einfluss hatte.

Ein Grund für das insignifikante Ergebnis der Gesamtmortalität könnte die zu kleine Studienkohorte in dieser Untersuchung sein. Es ist denkbar, dass die Nachbeobachtung einer größeren Patientengruppe ein signifikantes Ergebnis geliefert hätte, wie es in anderen Fällen mit 1250 Studienteilnehmern (Barefoot et al. 2000), mit 887 Studienteilnehmern (Frasure-Smith et al. 2000) oder mit 1234 Studienteilnehmern (Damen et al. 2013) der Fall war. Man könnte 
argumentieren, dass die Ereigniszahlen im Nachbeobachtungszeitraum zu gering waren. In diesem Fall wäre diese Studie nicht aussagekräftig genug (underpowered), um den signifikanten Effekt der Depressivität auf die Gesamtmortalität darzustellen.

Dennoch konnte genau dieser Effekt für ein Follow-Up-Intervall von 2 Jahren nachgewiesen werden. Man könnte anhand dieses Unterschieds schlussfolgern, dass der Zusammenhang kurz nach PCI vorhanden ist, sich aber im weiteren Verlauf abschwächt. Andere Faktoren wie Alter oder Komorbiditäten könnten in Langzeitbeobachtungen die Wirkung der Depressivität überlagern, vergleichbar mit Studien, die Nachbeobachtungszeiträume von 8,1 und 5 Jahren betrachteten (Stewart et al. 2003; Kornerup et al. 2011).

Tatsächlich waren nach 5 Jahren anstatt der Depressivität nicht-psychische Faktoren wie Alter, Body-Mass-Index, Tabakabusus und Zustand nach Myokardinfarkt signifikante Einflussfaktoren der Gesamtmortalität. Ein vergleichbares Ergebnis lieferte die Studie von Parakh et al., die nach Einschluss eine signifikant erhöhte Sterblichkeitsrate Depressiver innerhalb der ersten 4 Monate beobachtete, die sich aber bei Betrachtung des Langzeitergebnisses verlor (Parakh et al. 2008).

Wie in der Einleitung bereits skizziert, könnten unterschiedliche Faktoren für die gesteigerte Sterblichkeit verantwortlich sein. Patienten mit einem höheren Grad an Depressivität könnten in Bezug auf ihr Gesundheitsverhalten ein nachlässigeres Verhalten aufweisen. Tatsächlich hatten die Studienteilnehmer in dieser Kohorte (wenn auch nicht signifikant) einen erhöhten Body-Mass-Index und neigten zu verstärktem Tabakabusus, was bekanntermaßen die Prognose stark verschlechtert. Depressivität mit der einhergehenden Antriebslosigkeit könnte zur Verringerung von körperlichen Aktivitäten führen und damit einer prognoseverbessernden sportlichen Betätigung im Weg stehen.

Des Weiteren könnten weniger gesundheitsbewusste Ernährungsgewohnheiten und der Verzehr ungesunder Lebensmittel durch die Freudlosigkeit forciert werden. Neben Nichteinhaltung von Diätverordnungen und verminderter Adhärenz bei der Einnahme von regelmäßig zu applizierenden Medikamenten könnte ein Mangel an Bewegung die Prognose dieser Patientenklientel verschlechtern (Ladwig et al. 2013). Wie oben erwähnt, gibt es im vorliegenden Datensatz zu diesen gesundheitsbezogenen Verhaltensweisen keine Angaben, sodass diese Annahmen nur hypothetischen Charakters sind. Genauso wenig weiß man über die HADS-Dpositive Subgruppe, ob sich das gesundheitsbezogene Patientenverhalten innerhalb des Nachbeobachtungszeitraums verbessert hat, etwa im Rahmen einer Remission der Depressivität, was die unterschiedliche Mortalitätsrate nach 2 bzw. nach 5 Jahren erklären könnte. 
Es ist belegt worden, dass die Depressivität neurophysiologische Prozesse wie die Aktivierung des sympathischen Nervensystems triggert. Folge dieser Aktivierung sind eine Erhöhung der Herzfrequenz und des Blutdrucks, was - genau wie die veränderte Kortisolausschüttung Einfluss auf die Prognose der Patienten hat (Vreeburg et al. 2009). Da der HADS-Fragebogen lediglich den momentanen Gemütszustand erfasst und keine überdauernden Charaktermerkmale misst, ist es durchaus vorstellbar, dass die beschriebenen neurophysiologischen Prozesse kurz nach Studieneinschluss Wirkung in Form von erhöhter Mortalität zeigten. Dieser Effekt könnte sich jedoch im Verlauf der Langzeitbeobachtung verlieren und durch andere, überdauernde Faktoren überlagert werden. Es ist denkbar, dass der auffällige Befund bei Studienbeginn bei einer Zahl von Patienten im Rahmen eines phasenhaften Verlaufs der Depression gemessen wurde. Im Fall einer eventuell im Anschluss stattgefundenen Remission würde sich folglich die Prognose verbessern. Auch eine prognoseverbessernde Wirkung von möglicherweise im Verlauf eingenommenen Antidepressiva wurde in dieser Studie nicht berücksichtigt und könnte zu einem Abschwächen des Effekts beigetragen haben.

Zusammengefasst konnten die Auswirkungen der Depressivität auf die Gesamtmortalität nur für einen Zeitraum von 2 Jahren, nicht aber für das vollständige Intervall von 5 Jahren gezeigt werden. Nachfolgende Studien sollten daher die Auswirkung der Depressivität für unterschiedliche Nachbeobachtungszeiträume analysieren.

\subsection{Diskussion der 2. Hypothese: Prognostische Effekte der Typ-D-Persönlichkeit}

Mittels DS14-Fragebogen wurde in dieser Studie untersucht, ob man anhand der Typ-DPersönlichkeit eine Vorhersage auf das weitere Krankheitsgeschehen und auf die Langzeitprognose nach PCI treffen kann. KHK-Patienten wurden dafür über einen 5-jährigen Nachbeobachtungszeitraum beobachtet, um anschließend einen Zusammenhang zu den aufgenommenen Endpunkten Gesamtmortalität, Major Adverse Cardiovascular Events und Revaskularisation herzustellen. Nach abgeschlossener Analyse konnte in dieser KHK-Patientenkohorte für das Typ-D-Muster kein prädiktiver Wert in Bezug auf die aufgenommen Endpunkte festgestellt werden. Im Gegenteil zeigte sich für die Gesamtmortalität und für das Auftreten eines Major Adverse Cardiovascular Events unerwartet eine geringfügig höhere Rate in der Gruppe ohne Typ-D-Persönlichkeit, wobei der Unterschied nicht signifikant war. Im Hinblick auf die aktuelle Literatur ordnet sich diese Studie in eine stetig wachsende Ansammlung von Publikationen ein, die keinen prognostischen Effekt der Typ-D-Persönlichkeit nachweisen konnten. Demnach stellt sich die Frage, ob die Kombination aus negativer Affektivität und sozialer 
Inhibition (bzw. deren Interaktionsterm) als psychologisches Konstrukt tatsächlich ein valides Maß zur Ermittlung des kardialen Verlaufs der Patienten darstellt.

In dieser Studienkohorte zeigten sich keinerlei Zusammenhänge zwischen der Typ-DPersönlichkeit und den Endpunkten Mortalität, Major Adverse Cardiovascular Events und Revaskularisation, wobei weder univariate noch multivariate Analysen signifikante Ergebnisse lieferten. Diese Erkenntnis ist vor allem deswegen bemerkenswert, da die Häufigkeit der beiden wichtigsten kardiovaskulären Risikofaktoren Tabakabusus und Hypercholesterinämie in der Typ-D-Subgruppe signifikant höher war. Während unabhängige statistische Effekte von Rauchen und Zustand nach Myokardinfarkt bei Einschluss auf das Langzeituiberleben nachgewiesen werden konnten, zeigte sich kein nachteiliger Effekt des Typ-D-Musters auf die Gesamtmortalität. Auch weitere Berechnungen mit kontinuierlichen Werten der negativen Affektivität, der sozialen Inhibition sowie deren Interaktionsterm, anstatt der dichotomen Typ-D-Variablen, ergaben keine signifikanten Ergebnisse.

In dieser Studienkohorte sind nicht die Typ-D-Merkmale, sondern die gut etablierten Risikofaktoren positiv mit dem kardiovaskulären Langzeitergebnis der Patienten assoziiert. Komplikationen im Sinne von Revaskularisationsmaßnahmen waren zwar etwas häufiger bei Patienten mit Typ-D-Persönlichkeit, erreichten jedoch keine Signifikanz.

Alle bisherigen Studien, die einen ungünstigen Effekt des Typ-D nahelegten, stammten aus der Arbeitsgruppe um Denollet in Antwerpen und Tilburg. Eine aktuelle Metaanalyse (Grande et al. 2012) konnte aber zeigen, dass die Effekte mit dem Zeitpunkt der Publikationen abnahmen. Selbst die Arbeitsgruppe aus Tilburg konnte in neueren Studien keine prognostische Bedeutung des Typ-D-Musters nachweisen (Pelle et al. 2010, Damen et al. 2013). So wurden z.B. 1234 konsekutive PCI-Patienten aus dem, Rapamycin-Eluting Stent Evaluated At Rotterdam Cardiology Hospital (RESEARCH)' Register eingeschlossen (Damen et al. 2013). Nach Adjustierung für Typ-D zeigte sich innerhalb des 7-jährigen Nachbeobachtungszeitraums ein 1,6-fach erhöhtes Mortalitätsrisiko für depressive Patienten. Für die Typ-DPersönlichkeit konnten die Autoren keinen direkten Zusammenhang zur Gesamtmortalität herstellen. In Anlehnung an diese Daten konnte auch in der vorliegenden Studie kein prognostischer Wert für Typ-D ermittelt werden. Gleichartige Resultate in Bezug auf die Mortalitätsrate bei Herzinsuffizienzpatienten legten zwei kürzlich veröffentlichte Studien dar, eine mit 641 Studienteilnehmern, die andere mit 111 (Pelle et al. 2010, Volz et al. 2011). Eine weitere Studie, die eine heterogene Stichprobe von 1040 kardiologischen Patienten aus unterschiedlichen kardiologischen Settings untersuchte, fand ebenfalls einen Nulleffekt der Typ-D- 
Persönlichkeit auf die Mortalität (Grande et al. 2011). Die Metaanalyse von Grande et al. (2012) untersuchte prospektive Typ-D-Studien mit harten Endpunkten und fand dabei heraus, dass dieses Persönlichkeitskonstrukt evtl. nur die Prognose von KHK-Patienten, nicht jedoch diejenige von Patienten mit chronischer Herzinsuffizienz beeinflusst. Angesichts der vorliegenden Daten und der Studie von Damen et al. (2013) scheint selbst diese Schlussfolgerung zweifelhaft zu sein.

Negative Affektivität kombiniert mit sozialer Inhibition erfasst einen heterogenen emotionalen Zustand, der sich von anderen emotionalen Konstrukten wie Angst und Depressivität unterscheidet (Denollet et al. 2009c). Menschen mit hohen Werten untersuchen ihre soziale Umgebung auf für sie bedrohlich wirkende Situationen und reagieren empfindlicher auf zwischenmenschlichen Stress. Die Subskalen des DS14 registrieren demnach globale Persönlichkeitsmerkmale, ohne dabei primär eine prognostisch relevante Aussage auf das weitere Krankheitsgeschehen $\mathrm{zu}$ treffen. Vielmehr stellten vorangegangene Studien Korrelationen zum Neurotizismus und zur Introversion fest (Denollet et al. 2005). Typ-D bietet demnach weder einen Erklärungsansatz für die Ätiologie der KHK, noch bezieht sich dieses Konstrukt auf ein konsistentes neurobiologisches Substrat für selbsteingeschätzte negative Antwortstile (Ormel et al. 2004; Coyne und de Voogd 2012).

In dieser Studienkohorte waren die Patienten in der Subgruppe mit Typ-D-Persönlichkeit signifikant jünger als die Teilnehmer ohne Typ-D-Muster. Das könnte dazu führen, dass sowohl die Mortalitätsrate als auch die Rate an kardiovaskulären Komplikationen durch diesen Zusammenhang beeinflusst wird. Jedoch war auch nach Alterskontrolle in dieser Studienkohorte kein Effekt des Typ-D ausfindig zu machen. Der höhere Prozentsatz an Typ-DPersönlichkeiten bei jüngeren Menschen wurde ebenfalls in einer großen Untersuchung an einer Bevölkerungsstichprobe aus dem Rhein-Main-Gebiet mit 5000 Personen zwischen 35 und 74 Jahren festgestellt (Beutel et al. 2012). Da im späten Erwachsenenalter die Häufigkeit der Typ-D-Persönlichkeit in beiden Geschlechtern abzunehmen scheint, lässt sich die anfängliche Annahme, dass es sich um ein stabiles Persönlichkeitsmerkmal handelt, nicht aufrechterhalten. Eine andere Erklärung wäre, dass Individuen mit einer Typ-D-Persönlichkeit früher sterben und Patienten ohne Typ-D dadurch im höheren Alter überrepräsentiert sind. Es konnte jedoch belegt werden, dass die Typ-D-Werte auch unter den Überlebenden einer 5-JahresBeobachtungsstudie nicht wesentlicher stabiler waren als die HADS-Werte (Romppel et al. 2012). 
Zusammenfassend zeigt die vorliegende Studie, dass eine Einteilung in eine Typ-DPersönlichkeit keinen prädiktiven Wert bezüglich des klinischen Ergebnisses der KHKPatienten nach sich zieht. Trotz eines signifikant höheren Aufkommens von etablierten kardiovaskulären Risikofaktoren wie Tabakabusus oder Zustand nach Myokardinfarkt in der Typ-D-Subgruppe, unterschied sich die Mortalität nicht zwischen den beiden Fraktionen. Des Weiteren traten bei den Patienten mit Typ-D-Muster eher weniger Major Adverse Cardiovascular Events auf, auch wenn der Unterschied nicht statistisch signifikant war.

Das Misslingen, mithilfe des DS14-Fragebogens in dieser typischen KHKPatientenstichprobe ein erhöhtes kardiovaskuläres Risiko zu bestimmen, nährt auch existierende Zweifel über die psychopathologische Gültigkeit des Typ-D-Konstrukts (z.B. Smith 2011, Coyne und de Voogd 2012). Es fällt auf, dass bisher nur in niederländischen und in belgischen Studien einer einzigen Arbeitsgruppe ein signifikanter Einfluss der Typ-DPersönlichkeit auf das Überleben festgestellt werden konnte. Um eine mögliche kulturelle Überformung dieses Zusammenhangs aufzudecken, sollten psychokardiologische Typ-DStudien in weiteren Ländern durchgeführt werden. Die existierenden Studien aus dem deutschen Sprachraum stützen das Konzept von Denollet et al. jedenfalls nicht. Zukünftige Untersuchungen auf der Grundlage von narrativen Patienteninterviews und psychologischen Beurteilungen sollten demnach zum Ziel haben, die methodischen Defizite der willkürlich dichotomisierten und wahrscheinlich mit der DS14 auch unpräzise abgebildeten Typ-DPersönlichkeit zu überwinden, ehe darüber entschieden werden kann, ob sich dieses Konzept insgesamt als Prognosemarker überholt hat.

\subsection{Diskussion der 3. Hypothese: Prognostische Effekte der Angst}

Mittels HADS-Angstskala wurde die Angst mit der Gesamtmortalität, mit dem Auftreten von Major Adverse Cardiovascular Events und der Revaskularisationsrate nach PCI in Zusammenhang gebracht. Es zeigte sich ein signifikant verbessertes Überleben ängstlicher Patienten innerhalb der 5 Jahre nach durchgeführter PCI. Die vorteilhaften Effekte der erhöhten Angst auf kardiovaskuläre Morbidität und Mortalität zeigten sich unabhängig von zahlreichen, etablierten, kardiovaskulären Risikofaktoren und somatischen Kovariaten. Es stellte sich folglich heraus, dass die selbst eingeschätzte Angst ein vorteilhaftes klinisches Ergebnis vorhersagte.

Ebenso zeigte sich, dass ein erhöhter Grad an Angst bei Einschluss mit einem erniedrigten Risiko für das Auftreten eines Major Adverse Cardiovascular Events innerhalb von 5 Jahren nach PCI assoziiert war. Auf eine direkte Kausalität dieser deskriptiven Verhalte lässt sich auf 
Grundlage der Studie nur eingeschränkt schließen, da die begründenden klinischen Mechanismen überwiegend nicht bekannt sind und daher Raum für Diskussion schaffen. Der protektive Effekt zeigte sich bei den ängstlichen Patienten mit einer Latenz von ca. 700 Tagen zeitlich versetzt, vermutlich da die Wirkung von Therapievorschlägen, die bei Einschluss gegeben wurden, verspätet eingesetzt haben könnte (vgl. Abb. 18). Solche Therapievorschläge wurden häufig in den Patientenakten dokumentiert und beinhalteten meist Empfehlungen wie z.B. Gewichtsreduktion durch Bewegung, Umstellung der Ernährung oder Aufgabe von Tabakabusus. Dass diese sekundärpräventiven Maßnahmen zu einer deutlichen Prognoseverbesserung führen (v.a. Tabakabstinenz) ist für die meisten der etablierten Risikofaktoren bekannt. Das unter dem Eindruck der PCI initiierte angstbedingt bessere Gesundheitsverhalten könnte zunächst noch wenig Auswirkungen haben, da Ernährungsumstellung und körperliche Betätigung die Prognose nicht sofort sondern mit Verzögerung verändert haben könnten. Umgekehrt könnte die akute Verängstigung durch die Notwendigkeit der PCI über eine gewisse Zeit zu einer autonomen Imbalance geführt haben, die das kardiale Risiko im gleichen Maße erhöht wie das bessere Gesundheitsverhalten sie reduziert. Auf längere Sicht könnten sich viele Patienten an die neue Lebenssituation gewöhnen, die Angst würde im weiteren Verlauf abnehmen aber das günstige Verhalten, das inzwischen zur Routine geworden sein könnte, würde evtl. beibehalten, sodass seine Folgen auf lange Sicht überwiegen könnten. Es erscheint demnach wahrscheinlich, dass ängstliche Patienten besorgter um ihren Gesundheitszustand sein könnten und somit empfänglicher für medizinische Ratschläge wären als nicht-ängstliche Patienten, was sich häufig mit Erfahrungen der klinischen Praxis deckt. Das gesundheitsfördernde Verhalten ängstlicher Patienten könnte so zur Einhaltung dieser sekundärpräventiven Maßnahmen wie Lebensstiländerung, regelmäßige Einnahme von Mitteln zum Senken der Blutfettwerte und disziplinierteres Umsetzen einer angeordneten dualen Plättchenhemmung nach PCI führen. Das vorbeugende Verhalten der ängstlichen Patientengruppe könnte so zum einen eine positive Auswirkung auf die Morbidität und die Mortalität zur Folge haben und zum anderen die Rate schwerwiegender kardiovaskulärer Ereignisse (MACEs) signifikant senken.

Im Gegenzug könnte der Verzicht auf ebensolche medizinische Maßnahmen bei nicht oder weniger ängstlichen Patienten mit einem erhöhten Grad von Krankheitsverleugnung einhergehen. Diese könnte dann einer angemessenen Kontrolle der Risikofaktoren im Weg stehen, was letztendlich dazu führen könnte, dass rechtzeitige Diagnosestellung und angemessene Behandlung im Fall tödlicher Komplikationen hinausgezögert würden. In diesem Fall könnte ein eher verleugnender Bewältigungsstil zu einer höheren Gefährdung gerade bei den Patien- 
ten führen, die im Fragebogen eine niedrige Angst angaben und somit die erhöhte Mortalitätsrate und die gesteigerte Rate an kardiovaskulären Events in dieser Patientengruppe erklären. Bei expliziter Betrachtung der Quartile lässt sich dieser Zusammenhang jedoch nur eingeschränkt belegen, da sich kein besonders erhöhtes Risiko in der am wenigsten ängstlichen Gruppe zeigte. Vielmehr war die Gesamtmortalität und die Rate an MACEs im jeweils zweiten Quartil am höchsten.

Einen weiteren Erklärungsansatz liefert eine Studie, die einen unabhängigen und inversen Zusammenhang von Angst und gemessenen Werten des Herzinsuffizienzmarkers NT-proANP beschreibt. Es wurde dargestellt, dass mehr Angst das Vorliegen niedrigerer natriuretischer Peptidspiegel anzeigt, die ihrerseits prognostisch günstig sind (Herrmann-Lingen et al. 2003). Bei Patienten der hier untersuchten Studienkohorte kann ein hoher Angstwert evtl. analog als Indikator einer minder schweren funktionellen Ausprägung einer Herzinsuffizienz interpretiert werden und dadurch mit einer besseren Prognose assoziiert sein.

Tatsächlich spiegelte sich das angstgetriebene Verhalten der HADS-A-positiven Patientengruppe möglicherweise in der signifikant erhöhten Rate der Revaskularisationsmaßnahmen wider, was sich dadurch erklären ließe, dass diese Patienten ein größeres Verlangen nach medizinischen Interventionen haben könnten. Bekanntermaßen haben ängstliche Patienten insgesamt ein verstärktes Bedürfnis nach medizinischer Behandlung, verglichen mit weniger ängstlichen Patienten, die unter gleichen oder sogar gravierenderen Beschwerden leiden. Es scheint nachvollziehbar, dass ängstliche Patienten bereits bei geringer Symptomausprägung einen Arzt aufsuchen, ihre Beschwerden betonter vorbringen und dem Arzt damit aggressivere Therapien, u.a. in Form von Revaskularisationsmaßnahmen, nahelegen. Interessanterweise hat die Meta-Analyse von Roest et al. in Postmyokardinfarktpatienten signifikant größere Effekte der Angst auf das kardiale Outcome gezeigt (inklusive Revaskularisation), als auf die kardiale Mortalität allein. Dabei betrug die zusammengefasste Odds Ratio beim Vergleich von 579 ängstlichen mit 1075 nicht-ängstlichen Myokardinfarktpatienten für kardiale Events 1,71 (95\% CI=1,31-2,23, p<0,001). In Bezug auf die kardiale Mortalität lag die zusammengefasste Odds Ratio bei der Gegenüberstellung von 265 ängstlichen mit 1072 nicht-ängstlichen Patienten bei 1,23 (95\% CI=1,03-1,47, p<0,02). Dabei wurden die Daten in dichotomisierte Outcomes konvertiert und anschließend unadjustiert auf Signifikanz getestet (Roest et al. 2010b).

Ein gewisser Grad an Angst scheint sich protektiv auf das weitere Krankheitsgeschehen und insbesondere auf die Mortalität auszuwirken. Diesen positiven Effekt gilt es, sofern er sich bestätigt, dem Patienten zugutekommen zu lassen, ohne dass er sich durch seine Angst im 
Alltag zu sehr eingeschränkt fühlt. So könnte eine besondere Aufklärung bezüglich der Prognose der Angst mit anschließend gezieltem Shared Decision Making (engl.: Beteiligung des Patienten bei Entscheidungsfindung bezüglich Therapieverlauf) das weitere Krankheitsgeschehen des Patienten günstig beeinflussen und zu weniger schwerwiegenden kardiovaskulären Ereignissen führen. Demzufolge sollte bei der KHK gegebenenfalls die Diagnosestellung und die Behandlung der Angst mitbedacht werden. Die Herausforderung für den Therapeuten ist es dabei, die Angst des Patienten zu dessen Vorteil zu nutzen, ohne dabei die Lebensqualität zu verschlechtern.

Diese Erkenntnisse bringen vorausgegangene Studien, die sich im Aspekt der prognostischen Relevanz der Angst bei kardiovaskulären Patienten widersprechen insofern in einen neuen Kontext, als dass in dieser Kohorte ein weiteres Mal der protektive Effekt der Angst auf den Krankheitsverlauf der KHK-Patienten belegt wurde. Auf der einen Seite wurde gezeigt, dass die Angst ein klinisch relevanter Risikofaktor für das Auftreten von Kurz- und Langzeitkomplikationen nach Myokardinfarkt ist (Frasure-Smith et al. 1995b; Albert et al. 2005; Moser et al. 2007; Roest et al. 2010b). Andere Studien wiederum kamen zu dem Ergebnis, dass die Angst einen günstigen Effekt für symptomatische Patienten mit stabiler KHK hat (Blumenthal et al. 1979; Herrmann et al. 2000; Meyer et al. 2010). Das gleiche Phänomen zeigte sich in der Allgemeinbevölkerung (Mykletun et al. 2007).

Aus diesem Sachverhalt lässt sich schlussfolgern, dass die Prognose ängstlicher Patienten unter Umständen durch die Schwere der Herzerkrankung bei Einschluss moduliert wird. Die Krankheitsentwicklung ängstlicher Individuen würde demzufolge nach Myokardinfarkt einen gravierenderen Verlauf nehmen als bei KHK-Patienten. Hierin decken sich die Ergebnisse der Studie von Meyer et al. (2010) mit den hier vorliegenden Resultaten. Die Resultate in Postmyokardinfarktpatienten könnten durch die häufig einhergehende Herzinsuffizienz mit ungleicher, ventrikulärer Kontraktilität erklärt werden. Pathophysiologisch wurde der Mechanismus, der zu einer Verschlechterung der Prognose führt mit einer Sympathikusaktivierung, einer veränderten Blutgerinnung durch Thrombozytenaktivierung und endothelialer Dysfunktion erklärt, welche insbesondere bei Postmyokardinfarktpatienten nachteilige Effekte auf myokardiale Regeneration haben könnten (Rozanski et al. 2005).

Ein anderes Bild zeigte sich jedoch bei der untersuchten Patientengruppe mit weniger schweren Krankheitsverläufen bzw. stabiler KHK. Die nachteiligen Mechanismen der angstinduzierten adrenergen Stimulation werden mutmaßlich durch ein verändertes Patientenverhalten kompensiert. Dieses äußert sich durch ein vermehrtes Bestreben, bei körperlichen Beschwer- 
den medizinische Maßnahmen in Anspruch zu nehmen, was sich hier in Form von signifikant erhöhten Revaskularisationsmaßnahmen in dieser Kohorte nachweisen ließ. Des Weiteren zeigten sich bei Studienbeginn innerhalb dieser KHK-Patientenkohorte Stenosen von über $70 \%$ in mehr als einem Koronargefäß vermehrt in der weniger ängstlichen Subgruppe verglichen mit den ängstlicheren Individuen. Unter Umständen könnte dieser Unterschied bereits Ausdruck eines stattgefundenen günstigeren Krankheitsverlaufs vor Studienbeginn sein, der sich dann über die folgenden fünf Jahre fortsetzte. Eine andere Erklärung wäre eine angstgetriebene frühere Inanspruchnahme von ärztlichen Maßnahmen. Die ängstlichen Patienten waren bei Einschluss signifikant jünger und sind evtl. bereits früher im Krankheitsverlauf zum Arzt gegangen, da sie ihre Symptome möglicherweise als bedrohlicher erlebt haben.

Ebenfalls ist denkbar, dass neben der Schwere der Herzerkrankung auch die Form der zugrundeliegenden Angst das Outcome der Patienten moduliert. In dieser Studie wurden leichtere Ausprägungen und Störungsformen in Form von allgemeinen Angstsymptomen erfasst, wie sie die HADS abbildet. Neben der Beschaffenheit der Angst (z.B. generalisiert oder phobisch) könnte auch die zeitliche Ausprägung der Angst (z.B. ständig besorgt oder anfallsartig auftretend) einen Einfluss auf die Prognose der Patienten haben. Für einen aussagekräftigeren Prognosevergleich der Patienten sollte demnach künftig auf die Gleichartigkeit der Angst bei Einschluss Wert gelegt werden. Selektionskriterien der Meta-Analyse von Roest et al. waren beispielsweise sowohl selbsteingeschätzte Angst als auch solche, die mittels klinischen Patienteninterviews erfasst wurde (Roest et al. 2010a).

Die vorliegende Studie zeigt, dass vermehrte Angst bei PCI-Patienten mit stabiler KHK einen klinisch relevanten Indikator für verbessertes Überleben darstellt, trotz des bekannten gegenteiligen Effekts auf die Lebensqualität. Erhöhte HADS-A-Werte gehen mit einem erniedrigten Risiko sowohl für den Tod als auch für das Auftreten eines Major Adverse Cardiovascular Events einher. Die erhöhte Revaskularisationsrate bei ängstlichen Patienten legt nahe, dass diese Patienten besonders empfänglich für gesundheitsbezogene Aspekte im Sinne von ärztlichen Handlungsweisen sind. Die Forderung nach medizinisch-interventionellen Maßnahmen könnte so vorrangig von eher ängstlichen Patienten ausgehen. Tatsächlich bekamen die Patienten aus der ängstlichsten Subgruppe bzw. aus dem vierten Quartil quantitativ am häufigsten erneut einen Koronarstent im Nachbeobachtungszeitraum implantiert.

Der Zusammenhang könnte mutmaßlich aus der vermehrten Inanspruchnahme von gesundheitsfördernden Maßnahmen resultieren, wobei die klinischen Mechanismen, die dieses Phänomen erklären, derzeit noch nicht bewiesen sind. Die Ergebnisse dieser Studie, die die vor- 
teilhaften Effekte der Angst auf die Gesamtmortalität und den vorfallsfreien Verlauf darstellen, belegen erneut, dass psychosoziale Faktoren bei der Langzeitrisikostratifikation bei KHK-Patienten nach PCI bedacht werden sollten. Die weitere Forschung auf diesem Gebiet sollte demnach herausfinden, ob ein Angstscreening mittels HADS-Fragebogen zur verbesserten Risikoeinschätzung hilfreich sein könnte. Zum jetzigen Zeitpunkt führen die beschriebenen Ergebnisse zu der Annahme, dass die Angst bei Patienten mit stabiler KHK sowohl günstige als auch nachteilige Effekte haben könnte. In jedem Fall scheint sie eine gewisse Bedeutung zu haben und sollte in Zukunft vermehrt Beachtung bei der Prognoseprüfung finden. 


\section{ZUSAMMENFASSUNG}

Der Einfluss der Angst, der Depressivität und der Typ-D-Persönlichkeit auf die Prognose herzkranker Menschen wurde in den vergangenen Jahren kontrovers diskutiert. Dabei zeigte sich für die Angst kein einheitliches Meinungsbild. Autoren sprachen auf der einen Seite von einem Risikofaktor, auf der anderen Seite wurde der protektive Einfluss dieses psychologischen Parameters nahegelegt. Die Depressivität hingegen wurde relativ einheitlich eher als Risikofaktor gesehen, wobei unterschiedliche Nachbeobachtungszeiträume untersucht wurden. Die Typ-D-Persönlichkeit hatte in niederländischen und belgischen Studien die Prognose kardial erkrankter Menschen negativ beeinflusst, was bisher aber nicht unabhängig bestätigt wurde. Eine Übertragbarkeit dieses Phänomens auf die hier untersuchte deutsche Studienkohorte sollte untersucht werden.

In diese Studie wurden 470 Patienten aus dem Klinikum Links der Weser nach PCI eingeschlossen. Die Teilnehmer füllten zu Studienbeginn den HADS-Fragebogen zur Messung von Angst und Depressivität und die DS14 zur Erfassung der Typ-D-Persönlichkeit aus. Bei 98,3\% der Patienten konnte eine vollständige Nachbeobachtung über 5 Jahre durchgeführt werden, wobei die Endpunkte Tod, Major Adverse Cardiovascular Events und Revaskularisationsmaßnahmen waren.

Für die depressiven Patienten war die Mortalitätsrate lediglich nach 2 Jahren signifikant erhöht $(\mathrm{HR}=4,29)$. Sowohl für den gesamten Nachbeobachtungszeitraum von 5 Jahren als auch für die anderen Endpunkte Major Adverse Cardiovascular Events und Revaskularisationsmaßnahmen konnten keine Zusammenhänge dargestellt werden ( $p>0,49)$. Möglicherweise wurde die Prognose HADS-D-positiver Patienten nur mittelfristig durch die Depressivität verschlechtert. Nach einigen Jahren könnte sich der Effekt abschwächen und durch andere, überdauernde Merkmale (z.B. Komorbiditäten) überlagert werden.

Für die Typ-D-Persönlichkeit konnte kein Zusammenhang zum weiteren Krankheitsverlauf und zur Prognose der Patienten nach PCI hergestellt werden. Mortalitätsrate ( $\mathrm{p}=0,37)$, Major Adverse Cardiovascular Events $(\mathrm{p}=0,564)$ und die Rate an Revaskularisationsmaßnahmen $(\mathrm{p}=0,8)$ wurden nicht durch das Vorhandensein dieses Persönlichkeitsmerkmals beeinflusst, was ernsthafte Zweifel an der prognostischen Glaubwürdigkeit dieses Konstrukts auslöst. Im Gegenteil verstarben (wenn auch nicht signifikant) mehr Studienteilnehmer ohne Typ-DMuster (9,3\% vs. 8,3\%), was klar im Widerspruch zu Denollets Ergebnissen steht. Die zukünftige Erforschung des Typ-D-Musters sollte sich auf weitere Länder und Ethnien ausdeh- 
nen um evtl. vorhandene kulturell bedingte Wechselbeziehungen zwischen der Typ-DPersönlichkeit und kardialen Endpunkten aufzudecken.

Für die Angst zeigte sich sowohl ein verbessertes Überleben $(\mathrm{HR}=0,21)$ der Patienten als auch eine erniedrigte Rate an Major Adverse Cardiovascular Events ( $\mathrm{HR}=0,34)$. Es ist anzunehmen, dass ängstliche Patienten nach PCI ein vermehrt gesundheitsförderndes Verhalten zeigten, sodass die Umsetzung sekundärpräventiver Maßnahmen prognoseverbessernd wirkte und die niedrigere Mortalitätsrate erklären könnte. Das erhöhte Bestreben, bei Beschwerden den Arzt aufzusuchen, könnte sich in Form der erhöhten Rate an Revaskularisationsmaßnahmen $(H R=1,62)$ widerspiegeln, da ängstliche Patienten bekanntermaßen ein vermehrtes Verlangen nach medizinischen Maßnahmen äußern.

Zusammenfassend zeigten sich unterschiedliche Effekte der gemessenen psychischen Parameter auf die Prognose der Patienten nach perkutaner Koronarintervention. Zukünftige Studien sollten die zugrundeliegenden Zusammenhänge des hier beschriebenen Einflusses weiter erforschen. 


\section{ANHANG}

Tabelle A.1: Cox-Regressionsanalyse mit ausgewählten Confoundern zur Berechnung der Gesamtmortalität in 5 Jahren Nachbeobachtung bei HADS-D $\geq 8$, p-Wert für das Gesamtmodell $=0,002$

\begin{tabular}{|c|c|c|c|}
\hline Kovariate & Hazard Ratio & $\begin{array}{c}\text { 95\%-Konfidenz- } \\
\text { intervall }\end{array}$ & p-Wert \\
\hline Geschlecht & 0,65 & $0,28-1,54$ & 0,33 \\
\hline Alter & 1,06 & $1,02-1,09$ & $0,003 * *$ \\
\hline Body-Mass-Index & 0,9 & $0,82-0,99$ & $0,027^{*}$ \\
\hline Hypertonie & 1,74 & $0,69-4,39$ & 0,242 \\
\hline Hypercholesterinämie & 0,68 & $0,34-1,37$ & 0,282 \\
\hline Diabetes mellitus & 1,19 & $0,56-2,53$ & 0,655 \\
\hline Tabakabusus & 2,46 & $1,18-5,13$ & $0,017^{*}$ \\
\hline Positive Familienanamnese & 1,18 & $0,61-2,29$ & 0,624 \\
\hline Z.n. Myokardinfarkt & 1,97 & $1,04-3,73$ & $0,036^{*}$ \\
\hline Z.n. Bypass-Operation & 1,7 & $0,7-4,16$ & 0,244 \\
\hline Stenttyp (DES) & 2,27 & $0,99-5,22$ & 0,054 \\
\hline Stentlänge & 1,0 & $0,97-1,03$ & 0,845 \\
\hline Stentdurchmesser & 1,19 & $0,53-2,7$ & 0,671 \\
\hline Proximale Stentlokalisation & 0,61 & $0,3-1,24$ & 0,171 \\
\hline Mehrgefäßerkrankung & 0,65 & $0,32-1,3$ & 0,225 \\
\hline HADS-D $\geq 8$ & 1,58 & $0,77-3,22$ & 0,214 \\
\hline
\end{tabular}

*signifikant auf dem 5\%-Niveau; **signifikant auf dem 1\%-Niveau; ***signifikant auf dem 0,1\%-Niveau 
Tabelle A.2: Cox-Regressionsanalyse mit ausgewählten Confoundern zur Berechnung des kumulativen ereignisfreien Überleben (MACE) in 5 Jahren Nachbeobachtung bei HADS$\mathrm{D} \geq 8, \mathrm{p}$-Wert für das Gesamtmodell $=0,002$

\begin{tabular}{|c|c|c|c|}
\hline Kovariate & Hazard Ratio & $\begin{array}{c}\text { 95\%-Konfidenz- } \\
\text { intervall }\end{array}$ & p-Wert \\
\hline Geschlecht & 0,85 & $0,45-1,6$ & 0,618 \\
\hline Alter & 1,03 & $1,0-1,06$ & $0,016^{*}$ \\
\hline Body-Mass-Index & 0,97 & $0,91-1,04$ & 0,462 \\
\hline Hypertonie & 1,16 & $0,59-2,27$ & 0,675 \\
\hline Hypercholesterinämie & 0,62 & $0,37-1,05$ & 0,78 \\
\hline Diabetes mellitus & 0,86 & $0,47-1,58$ & 0,631 \\
\hline Tabakabusus & 2,69 & $1,5-4,84$ & $0,001 * *$ \\
\hline Positive Familienanamnese & 1,26 & $0,76-2,1$ & 0,369 \\
\hline Z.n. Myokardinfarkt & 1,78 & $1,07-2,95$ & $0,026^{*}$ \\
\hline Z.n. Bypass-Operation & 2,0 & $1,0-3,99$ & $0,048^{*}$ \\
\hline Stenttyp (DES) & 1,46 & $0,75-2,85$ & 0,272 \\
\hline Stentlänge & 1,0 & $0,99-1,03$ & 0,452 \\
\hline Stentdurchmesser & 1,21 & $0,64-2,29$ & 0,555 \\
\hline Proximale Stentlokalisation & 0,54 & $0,31-0,95$ & $0,032 *$ \\
\hline Mehrgefäßerkrankung & 0,87 & $0,5-1,49$ & 0,608 \\
\hline HADS-D $\geq 8$ & 0,94 & $0,51-1,73$ & 0,84 \\
\hline
\end{tabular}

*signifikant auf dem 5\%-Niveau; **signifikant auf dem 1\%-Niveau; ***signifikant auf dem 0,1\%-Niveau 
Tabelle A.3: Cox-Regressionsanalyse mit ausgewähltem Confounder zur Berechnung der Revaskularisationsrate in 5 Jahren Nachbeobachtung bei HADS-D $\geq 8$, p-Wert für das Gesamtmodell $=0,048$

\begin{tabular}{|l|c|c|c|}
\hline \multicolumn{1}{|c|}{ Kovariate } & Hazard Ratio & $\begin{array}{c}\text { 95\%-Konfidenz- } \\
\text { intervall }\end{array}$ & p-Wert \\
\hline Z.n. Bypass-Operation & 2,02 & $1,20-3,40$ & $0,008 * *$ \\
\hline HADS-D $\geq 8$ & 0,93 & $0,55-1,56$ & 0,77 \\
\hline
\end{tabular}

*signifikant auf dem 5\%-Niveau; **signifikant auf dem 1\%-Niveau; ***signifikant auf dem 0,1\%-Niveau 
Tabelle A.4: Cox-Regressionsanalyse mit ausgewählten Confoundern zur Berechnung der Revaskularisationsrate in 5 Jahren Nachbeobachtung bei HADS-D $\geq 8$, p-Wert für das Gesamtmodell $\mathrm{p}=0,303$

\begin{tabular}{|c|c|c|c|}
\hline Kovariate & Hazard Ratio & $\begin{array}{c}\text { 95\%-Konfidenz- } \\
\text { intervall }\end{array}$ & p-Wert \\
\hline Geschlecht & 0,88 & $0,52-1,48$ & 0,634 \\
\hline Alter & 0,98 & $0,96-1,01$ & 0,212 \\
\hline Body-Mass-Index & 1,01 & $0,95-1,07$ & 0,774 \\
\hline Hypertonie & 1,42 & $0,74-2,73$ & 0,287 \\
\hline Hypercholesterinämie & 0,94 & $0,58-1,52$ & 0,796 \\
\hline Diabetes mellitus & 1,53 & $0,95-2,47$ & 0,80 \\
\hline Tabakabusus & 1,06 & $0,59-1,88$ & 0,847 \\
\hline Positive Familienanamnese & 1,15 & $0,73-1,80$ & 0,545 \\
\hline Z.n. Myokardinfarkt & 0,92 & $0,55-1,52$ & 0,734 \\
\hline Z.n. Bypass-Operation & 2,44 & $1,32-4,49$ & $0,004 * *$ \\
\hline Stenttyp (DES) & 0,73 & $0,39-1,36$ & 0,326 \\
\hline Stentlänge & 1,0 & $0,97-1,02$ & 0,848 \\
\hline Stentdurchmesser & 0,55 & $0,29-1,03$ & 0,061 \\
\hline Proximale Stentlokalisation & 1,21 & $0,77-1,90$ & 0,407 \\
\hline Mehrgefäßerkrankung & 0,91 & $0,56-1,49$ & 0,720 \\
\hline HADS-D $\geq 8$ & 0,83 & $0,48-1,42$ & 0,492 \\
\hline
\end{tabular}

*signifikant auf dem 5\%-Niveau; **signifikant auf dem 1\%-Niveau; ***signifikant auf dem 0,1\%-Niveau 
Tabelle A.5: Cox-Regressionsanalyse mit ausgewählten Confoundern zur Berechnung der Gesamtmortalität in 2 Jahren Nachbeobachtung bei HADS-D $\geq 8$, p-Wert für das Gesamtmodell $=0,027$

\begin{tabular}{|c|c|c|c|}
\hline Kovariate & Hazard Ratio & $\begin{array}{c}\text { 95\%-Konfidenz- } \\
\text { intervall }\end{array}$ & p-Wert \\
\hline Geschlecht & 0,16 & $0,02-1,47$ & 0,105 \\
\hline Alter & 0,98 & $0,92-1,04$ & 0,499 \\
\hline Body-Mass-Index & 0,82 & $0,68-0,99$ & $0,040^{*}$ \\
\hline Hypertonie & 3,02 & $0,55-16,62$ & 0,203 \\
\hline Hypercholesterinämie & 0,40 & $0,10-1,64$ & 0,202 \\
\hline Diabetes mellitus & 2,66 & $0,57-12,38$ & 0,213 \\
\hline Tabakabusus & 2,99 & $0,76-11,82$ & 0,118 \\
\hline Positive Familienanamnese & 1,13 & $0,32-3,91$ & 0,851 \\
\hline Z.n. Myokardinfarkt & 1,44 & $0,40-5,21$ & 0,579 \\
\hline Z.n. Bypass-Operation & 3,54 & $0,58-21,44$ & 0,169 \\
\hline Stenttyp (DES) & 2,78 & $0,58-13,42$ & 0,202 \\
\hline Stentlänge & 1,06 & $1,00-1,11$ & $0,042 *$ \\
\hline Stentdurchmesser & 0,25 & $0,04-1,68$ & 0,15 \\
\hline Proximale Stentlokalisation & 0,48 & $0,09-2,43$ & 0,375 \\
\hline Mehrgefäßerkrankung & 0,25 & $0,05-1,21$ & 0,085 \\
\hline HADS-D $\geq 8$ & 4,29 & $1,20-15,39$ & $0,025 *$ \\
\hline
\end{tabular}

*signifikant auf dem 5\%-Niveau; **signifikant auf dem 1\%-Niveau; ***signifikant auf dem 0,1\%-Niveau 
Tabelle A.6: Gruppenvergleich der Patienten mit und ohne HADS-D $\geq 8$

\begin{tabular}{|c|c|c|c|c|}
\hline Kovariate & $\begin{array}{c}\text { Totale Ko- } \\
\text { horte } \\
(\mathrm{N}=\mathbf{4 7 0})\end{array}$ & $\begin{array}{c}\text { HADS-D<8 } \\
(\mathrm{N}=369)\end{array}$ & $\begin{array}{c}\text { HADS-D } \geq 8 \\
(\mathrm{~N}=101)\end{array}$ & $\begin{array}{l}\text { Gruppen- } \\
\text { vergleich } \\
\text { (p-Wert) }\end{array}$ \\
\hline Geschlecht (männlich) & $361(76,8 \%)$ & $288(78 \%)$ & $73(72,3 \%)$ & 0,233 \\
\hline Alter (Jahre) (SD) & $63,7(9,9)$ & $64,4(9,6)$ & $61,2(10,4)$ & $0,003 * *$ \\
\hline Body-Mass-Index (SD) & $28(4)$ & $27,8(3,8)$ & $28,4(4,5)$ & 0,207 \\
\hline Hypertonie & $380(80,8 \%)$ & $303(82,1 \%)$ & $77(76,2 \%)$ & 0,192 \\
\hline Hypercholesterinämie & $318(67,6 \%)$ & $250(67,7 \%)$ & $68(67,3 \%)$ & 1,0 \\
\hline Diabetes mellitus & $111(23,6 \%)$ & $87(23,6 \%)$ & $24(23,8 \%)$ & 1,0 \\
\hline Tabakabusus & $103(21,9 \%)$ & $74(20,1 \%)$ & $29(28,7 \%)$ & 0,076 \\
\hline Positive Familienanamnese & $181(38,5 \%)$ & $138(37,4 \%)$ & $43(42,6 \%)$ & 0,355 \\
\hline Z.n. Myokardinfarkt & $125(26,6 \%)$ & $98(26,6 \%)$ & $27(26,7 \%)$ & 1,0 \\
\hline Z.n. Bypass-Operation & $57(12,1 \%)$ & $41(11,1 \%)$ & $16(15,8 \%)$ & 0,228 \\
\hline Stenttyp (DES) & $(15,5 \%)$ & $(16,3 \%)$ & $(13,9 \%)$ & 0,852 \\
\hline Stentlänge (mm) (SD) & $19,9(9,9)$ & $20(9,8)$ & $19,5(10,2)$ & 0,637 \\
\hline Stentdurchmesser (mm) (SD) & $2,9(0,4)$ & $2,9(0,4)$ & $2,9(0,4)$ & 0,969 \\
\hline Proximale Stentlokalisation & $186(39,6 \%)$ & $139(37,7 \%)$ & $47(46,5 \%)$ & 0,135 \\
\hline Mehrgefäßerkrankung & $242(51,5 \%)$ & $195(52,8 \%)$ & $47(46,5 \%)$ & 0,264 \\
\hline HADS-D bei Einschluss & $5,1(3,4)$ & $3,7(2,1)$ & $10,1(2,1)$ & $<0,001 * * *$ \\
\hline Mortalität & $44(9,4 \%)$ & $33(8,9 \%)$ & $11(10,9 \%)$ & 0,561 \\
\hline MACE & $73(15,5 \%)$ & $57(15,4 \%)$ & $16(15,8 \%)$ & 1,0 \\
\hline Revaskularisation & $87(18,5 \%)$ & $69(18,7 \%)$ & $18(17,8 \%)$ & 0,886 \\
\hline
\end{tabular}

*signifikant auf dem 5\%-Niveau; **signifikant auf dem 1\%-Niveau; ***signifikant auf dem 0,1\%-Niveau 
Tabelle B.1: Cox-Regressionsanalyse mit ausgewählten Confoundern zur Berechnung der Gesamtmortalität in 5 Jahren Nachbeobachtung beim Vorliegen einer Typ-D-Persönlichkeit, p-Wert für das Gesamtmodell=0,001

\begin{tabular}{|c|c|c|c|}
\hline Kovariate & Hazard Ratio & $\begin{array}{c}\text { 95\%-Konfidenz- } \\
\text { intervall }\end{array}$ & p-Wert \\
\hline Geschlecht & 0,69 & $0,29-1,62$ & 0,387 \\
\hline Alter & 1,05 & $1,01-1,09$ & $0,009 * *$ \\
\hline Body-Mass-Index & 0,90 & $0,82-0,99$ & $0,037 *$ \\
\hline Hypertonie & 1,76 & $0,7-4,47$ & 0,232 \\
\hline Hypercholesterinämie & 0,67 & $0,33-1,35$ & 0,263 \\
\hline Diabetes mellitus & 1,15 & $0,54-2,46$ & 0,724 \\
\hline Tabakabusus & 2,42 & $1,13-5,19$ & $0,023^{*}$ \\
\hline Positive Familienanamnese & 1,18 & $0,61-2,28$ & 0,625 \\
\hline Z.n. Myokardinfarkt & 1,93 & $1,02-3,66$ & $0,043^{*}$ \\
\hline Z.n. Bypass-Operation & 1,81 & $0,74-4,42$ & 0,193 \\
\hline Stenttyp (DES) & 2,07 & $0,9-4,78$ & 0,088 \\
\hline Stentlänge & 1,0 & $0,97-1,04$ & 0,808 \\
\hline Stentdurchmesser & 1,19 & $0,53-2,71$ & 0,675 \\
\hline Proximale Stentlokalisation & 0,59 & $0,29-1,2$ & 0,144 \\
\hline Mehrgefäßerkrankung & 0,64 & $0,32-1,28$ & 0,206 \\
\hline Typ-D & 1,09 & $0,91-1,31$ & 0,367 \\
\hline
\end{tabular}

*signifikant auf dem 5\%-Niveau; **signifikant auf dem 1\%-Niveau; ***signifikant auf dem 0,1\%-Niveau 
Tabelle B.2: Cox-Regressionsanalyse mit ausgewählten Confoundern zur Berechnung des kumulativen ereignisfreien Überlebens (MACE) in 5 Jahren Nachbeobachtung beim Vorliegen einer Typ-D-Persönlichkeit, $\mathrm{p}$-Wert für das Gesamtmodell=0,001

\begin{tabular}{|c|c|c|c|}
\hline Kovariate & Hazard Ratio & $\begin{array}{c}\text { 95\%-Konfidenz- } \\
\text { intervall }\end{array}$ & p-Wert \\
\hline Geschlecht & 0,85 & $0,45-1,61$ & 0,624 \\
\hline Alter & 1,03 & $1,01-1,06$ & $0,019 *$ \\
\hline Body-Mass-Index & 0,98 & $0,91-1,05$ & 0,477 \\
\hline Hypertonie & 1,17 & $0,59-2,31$ & 0,648 \\
\hline Hypercholesterinämie & 0,63 & $0,37-1,06$ & 0,082 \\
\hline Diabetes mellitus & 0,85 & $0,46-1,56$ & 0,603 \\
\hline Tabakabusus & 2,57 & $1,42-4,64$ & $0,002 * *$ \\
\hline Positive Familienanamnese & 1,26 & $0,76-2,09$ & 0,378 \\
\hline Z.n. Myokardinfarkt & 1,77 & $1,07-2,93$ & $0,027 *$ \\
\hline Z.n. Bypass-Operation & 1,98 & $1,0-3,91$ & $0,05 *$ \\
\hline Stenttyp (DES) & 1,44 & $0,74-2,82$ & 0,283 \\
\hline Stentlänge & 1,01 & $0,99-1,03$ & 0,431 \\
\hline Stentdurchmesser & 1,22 & $0,65-2,32$ & 0,539 \\
\hline Proximale Stentlokalisation & 0,53 & $0,30-0,93$ & $0,028^{*}$ \\
\hline Mehrgefäßerkrankung & 0,86 & $0,50-1,48$ & 0,593 \\
\hline Typ-D & 1,05 & $0,88-1,26$ & 0,564 \\
\hline
\end{tabular}

*signifikant auf dem 5\%-Niveau; **signifikant auf dem 1\%-Niveau; ***signifikant auf dem 0,1\%-Niveau 
Tabelle B.3: Cox-Regressionsanalyse mit ausgewählten Confoundern zur Berechnung der Revaskularisationsrate in 5 Jahren Nachbeobachtung beim Vorliegen einer Typ-DPersönlichkeit, $\mathrm{p}$-Wert für das Gesamtmodell $\mathrm{p}=0,319$

\begin{tabular}{|c|c|c|c|}
\hline Kovariate & Hazard Ratio & $\begin{array}{c}\text { 95\%-Konfidenz- } \\
\text { intervall }\end{array}$ & p-Wert \\
\hline Geschlecht & 0,87 & $0,52-1,46$ & 0,590 \\
\hline Alter & 0,98 & $0,96-1,01$ & 0,223 \\
\hline Body-Mass-Index & 1,01 & $0,95-1,07$ & 0,809 \\
\hline Hypertonie & 1,44 & $0,75-2,76$ & 0,273 \\
\hline Hypercholesterinämie & 0,95 & $0,58-1,54$ & 0,832 \\
\hline Diabetes mellitus & 1,53 & $0,95-2,46$ & 0,083 \\
\hline Tabakabusus & 1,02 & $0,57-1,83$ & 0,933 \\
\hline Positive Familienanamnese & 1,14 & $0,73-1,79$ & 0,567 \\
\hline Z.n. Myokardinfarkt & 0,92 & $0,55-1,52$ & 0,737 \\
\hline Z.n. Bypass-Operation & 2,32 & $1,27-4,24$ & $0,006 * *$ \\
\hline Stenttyp (DES) & 0,74 & $0,40-1,38$ & 0,344 \\
\hline Stentlänge & 1,00 & $0,97-1,02$ & 0,849 \\
\hline Stentdurchmesser & 0,56 & $0,30-1,04$ & 0,068 \\
\hline Proximale Stentlokalisation & 1,18 & $0,75-1,86$ & 0,460 \\
\hline Mehrgefäßerkrankung & 0,92 & $0,56-1,49$ & 0,726 \\
\hline Typ-D & 1,03 & $0,83-1,27$ & 0,801 \\
\hline
\end{tabular}

*signifikant auf dem 5\%-Niveau; **signifikant auf dem 1\%-Niveau; ***signifikant auf dem 0,1\%-Niveau 
Tabelle B.4: Cox-Regressionsanalyse mit ausgewählten Confoundern zur Berechnung der Gesamtmortalität in 5 Jahren Nachbeobachtung bei Betrachtung der Rohwerte für NA und SI sowie deren Interaktionsterm, $\mathrm{p}$-Wert für das Gesamtmodell=0,033

\begin{tabular}{|c|c|c|c|}
\hline Kovariate & Hazard Ratio & $\begin{array}{c}\text { 95\%-Konfidenz- } \\
\text { intervall }\end{array}$ & p-Wert \\
\hline Geschlecht & 0,67 & $0,28-1,61$ & 0,372 \\
\hline Alter & 1,05 & $1,01-1,09$ & $0,011 *$ \\
\hline Body-Mass-Index & 0,9 & $0,82-1,0$ & $0,043 *$ \\
\hline Hypertonie & 1,95 & $0,72-5,23$ & 0,189 \\
\hline Hypercholesterinämie & 0,71 & $0,35-1,47$ & 0,361 \\
\hline Diabetes mellitus & 1,1 & $0,5-2,41$ & 0,816 \\
\hline Tabakabusus & 2,22 & $1,02-4,86$ & $0,045 *$ \\
\hline Positive Familienanamnese & 1,09 & $0,55-2,16$ & 0,799 \\
\hline Myokardinfarkt & 1,79 & $0,91-3,5$ & 0,089 \\
\hline Bypass-Operation & 1,92 & $0,78-4,71$ & 0,157 \\
\hline Stenttyp (DES) & 2,09 & $0,85-5,12$ & 0,107 \\
\hline Stentlänge & 1,01 & $0,97-1,04$ & 0,688 \\
\hline Stentdurchmesser & 1,19 & $0,52-2,74$ & 0,674 \\
\hline Proximale Stentlokalisation & 0,57 & $0,27-1,2$ & 0,138 \\
\hline Mehrgefäßerkrankung & 0,61 & $0,29-1,26$ & 0,180 \\
\hline DS14-NA & 1,02 & $0,91-1,15$ & 0,697 \\
\hline DS14-SI & 1,0 & $0,89-1,13$ & 0,963 \\
\hline DS14-NA*DS14-SI & 1,0 & $0,99-1,01$ & 0,976 \\
\hline
\end{tabular}

*signifikant auf dem 5\%-Niveau; **signifikant auf dem 1\%-Niveau; ***signifikant auf dem 0,1\%-Niveau 
Tabelle B.5: Cox-Regressionsanalyse mit ausgewählten Confoundern zur Berechnung der MACE in 5 Jahren Nachbeobachtung bei Betrachtung der Rohwerte für NA und SI sowie deren Interaktionterm, $\mathrm{p}$-Wert für das Gesamtmodell=0,003

\begin{tabular}{|c|c|c|c|}
\hline Kovariate & Hazard - Ratio & $\begin{array}{c}\text { 95\%-Konfidenz- } \\
\text { intervall }\end{array}$ & p-Wert \\
\hline Geschlecht & 0,78 & $0,41-1,49$ & 0,458 \\
\hline Alter & 1,04 & $1,01-1,07$ & $0,01^{*}$ \\
\hline Body-Mass-Index & 0,97 & $0,91-1,04$ & 0,434 \\
\hline Hypertonie & 1,19 & $0,59-2,4$ & 0,622 \\
\hline Hypercholesterinämie & 0,66 & $0,38-1,13$ & 0,13 \\
\hline Diabetes mellitus & 0,78 & $0,42-1,44$ & 0,426 \\
\hline Tabakabusus & 2,52 & $1,37-4,62$ & $0,003 * *$ \\
\hline Positive Familienanamnese & 1,25 & $0,74-2,1$ & 0,41 \\
\hline Z.n. Myokardinfarkt & 1,79 & $1,06-3,02$ & $0,03 *$ \\
\hline Z.n. Bypass-Operation & 2,24 & $1,12-4,47$ & $0,023^{*}$ \\
\hline Stenttyp (DES) & 1,36 & $0,67-2,75$ & 0,389 \\
\hline Stentlänge & 1,01 & $0,99-1,04$ & 0,301 \\
\hline Stentdurchmesser & 1,2 & $0,62-2,31$ & 0,58 \\
\hline Proximale Stentlokalisation & 0,52 & $0,29-0,92$ & $0,025^{*}$ \\
\hline Mehrgefäßerkrankung & 0,86 & $0,49-1,5$ & 0,59 \\
\hline DS14-NA & 1,04 & $0,96-1,13$ & 0,359 \\
\hline DS14-SI & 0,95 & $0,86-1,04$ & 0,282 \\
\hline DS14-NA*DS14-SI & 1,0 & $0,99-1,01$ & 0,756 \\
\hline
\end{tabular}

*signifikant auf dem 5\%-Niveau; **signifikant auf dem 1\%-Niveau; ***signifikant auf dem 0,1\%-Niveau 
Tabelle B.6: Cox-Regressionsanalyse mit ausgewählten Confoundern zur Berechnung der Revaskularisationsrate in 5 Jahren Nachbeobachtung bei Betrachtung der Rohwerte für NA und SI sowie deren Interaktionterm, p-Wert für das Gesamtmodell=0,387

\begin{tabular}{|c|c|c|c|}
\hline Kovariate & Hazard - Ratio & $\begin{array}{c}\text { 95\%-Konfidenz- } \\
\text { intervall }\end{array}$ & p-Wert \\
\hline Geschlecht & 0,85 & $0,50-1,44$ & 0,541 \\
\hline Alter & 0,99 & $0,96-1,01$ & 0,285 \\
\hline Body-Mass-Index & 1,00 & $0,94-1,06$ & 0,990 \\
\hline Hypertonie & 1,41 & $0,74-2,72$ & 0,297 \\
\hline Hypercholesterinämie & 0,92 & $0,56-1,50$ & 0,734 \\
\hline Diabetes mellitus & 1,44 & $0,89-2,35$ & 0,142 \\
\hline Tabakabusus & 1,07 & $0,59-1,91$ & 0,827 \\
\hline Positive Familienanamnese & 1,13 & $0,72-1,78$ & 0,592 \\
\hline Z.n. Myokardinfarkt & 0,96 & $0,57-1,60$ & 0,868 \\
\hline Z.n. Bypass-Operation & 2,45 & $1,33-4,52$ & $0,004 * *$ \\
\hline Stenttyp (DES) & 0,76 & $0,40-1,42$ & 0,389 \\
\hline Stentlänge & 1,00 & $0,97-1,02$ & 0,886 \\
\hline Stentdurchmesser & 0,54 & $0,29-1,02$ & 0,057 \\
\hline Proximale Stentlokalisation & 1,24 & $0,79-1,95$ & 0,352 \\
\hline Mehrgefäßerkrankung & 0,91 & $0,55-1,49$ & 0,706 \\
\hline DS14-NA & 1,00 & $0,92-1,07$ & 0,939 \\
\hline DS14-SI & 0,97 & $0,89-1,06$ & 0,493 \\
\hline DS14-NA*DS14-SI & 1,00 & $0,99-1,01$ & 0,664 \\
\hline
\end{tabular}

*signifikant auf dem 5\%-Niveau; **signifikant auf dem 1\%-Niveau; ***signifikant auf dem 0,1\%-Niveau 
Tabelle B.7: Gruppenvergleich der Patienten mit und ohne Typ-D-Persönlichkeit

\begin{tabular}{|c|c|c|c|c|}
\hline & $\begin{array}{c}\text { Totale Kohor- } \\
\text { te } \\
\quad(\mathrm{N}=\mathbf{4 6 5})\end{array}$ & $\begin{array}{l}\text { Keine Typ-D- } \\
\text { Persönlichkeit } \\
\qquad(\mathrm{N}=321)\end{array}$ & $\begin{array}{c}\text { Typ-D- } \\
\text { Persönlichkeit } \\
(\mathrm{N}=144)\end{array}$ & $\begin{array}{l}\text { Gruppen- } \\
\text { vergleich } \\
\text { (p-Wert) }\end{array}$ \\
\hline Geschlecht (männlich) & $356(76,6 \%)$ & $253(78,8 \%)$ & $103(71,5 \%)$ & 0,106 \\
\hline Alter (Jahre) (SD) & $63,7(9,9)$ & $64,3(9,2)$ & $61,9(10,9)$ & $0,005 * *$ \\
\hline Body-Mass-Index (SD) & $28(4)$ & $27,8(3,6)$ & $28,4(4,5)$ & 0,141 \\
\hline Hypertonie & $377(81,1 \%)$ & $261(81,3 \%)$ & $116(80,6 \%)$ & 0,413 \\
\hline Hypercholesterinämie & $315(67,7 \%)$ & $205(63,9 \%)$ & $110(76,4 \%)$ & $0,036^{*}$ \\
\hline Diabetes mellitus & $109(23,4 \%)$ & $75(23,4 \%)$ & $34(23,6 \%)$ & 0,692 \\
\hline Tabakabusus & $100(21,5 \%)$ & $61(19 \%)$ & $39(27,1 \%)$ & $0,02 *$ \\
\hline Positive Familienanamnese & $180(38,7 \%)$ & $117(36,4 \%)$ & $63(43,8 \%)$ & 0,250 \\
\hline Z.n. Myokardinfarkt & $123(26,5 \%)$ & $85(26,5 \%)$ & $38(26,4 \%)$ & 0,795 \\
\hline Z.n. Bypass-Operation & $56(12 \%)$ & $35(10,9 \%)$ & $21(14,6 \%)$ & 0,459 \\
\hline Stenttyp (DES) & $72(15,5 \%)$ & $54(16,8 \%)$ & $18(12,5 \%)$ & 0,714 \\
\hline Stentlänge (mm) (SD) & $19,9(9,9)$ & $20,1(10)$ & $19,4(9,8)$ & 0,498 \\
\hline $\begin{array}{l}\text { Stentdurchmesser (mm) } \\
\text { (SD) }\end{array}$ & $2,9(0,4)$ & $2,9(0,4)$ & $3(0,4)$ & 0,276 \\
\hline Proximale Stentlokalisation & $183(39,4 \%)$ & $120(37,4 \%)$ & $63(43,8 \%)$ & 0,287 \\
\hline Mehrgefäßerkrankung & $238(51,2 \%)$ & $164(51,1 \%)$ & $74(51,4 \%)$ & 0,439 \\
\hline Mortalität & $42(9 \%)$ & $30(9,3 \%)$ & $12(8,3 \%)$ & 0,861 \\
\hline MACE & $71(15,3 \%)$ & $51(15,9 \%)$ & $20(13,9 \%)$ & 0,676 \\
\hline Revaskularisation & $86(18,5 \%)$ & $58(18,1 \%)$ & $28(19,4 \%)$ & 0,936 \\
\hline
\end{tabular}

*signifikant auf dem 5\%-Niveau; **signifikant auf dem 1\%-Niveau; ***signifikant auf dem 0,1\%-Niveau 
Tabelle C.1: Cox-Regressionsanalyse mit ausgewählten Confoundern zur Berechnung der Gesamtmortalität in 5 Jahren Nachbeobachtung bei HADS-A $\geq 10$, p-Wert für das Gesamtmodell $<0,001$

\begin{tabular}{|c|c|c|c|}
\hline Kovariate & Hazard Ratio & $\begin{array}{c}\text { 95\%-Konfidenz- } \\
\text { intervall }\end{array}$ & p-Wert \\
\hline Geschlecht & 0,79 & $0,34-1,85$ & 0,586 \\
\hline Alter & 1,04 & $1,0-1,08$ & $0,035^{*}$ \\
\hline Body-Mass-Index & 0,90 & $0,82-0,99$ & $0,038^{*}$ \\
\hline Hypertonie & 1,75 & $0,7-4,42$ & 0,233 \\
\hline Hypercholesterinämie & 0,65 & $0,32-1,31$ & 0,232 \\
\hline Diabetes mellitus & 1,28 & $0,6-2,73$ & 0,518 \\
\hline Tabakabusus & 2,71 & $1,29-5,71$ & $0,009 * *$ \\
\hline Positive Familienanamnese & 1,2 & $0,62-2,34$ & 0,584 \\
\hline Z.n. Myokardinfarkt & 1,91 & $1,0-3,63$ & $0,048^{*}$ \\
\hline Z.n. Bypass-Operation & 2,04 & $0,8-5,18$ & 0,133 \\
\hline Stenttyp (DES) & 2,06 & $0,88-4,77$ & 0,094 \\
\hline Stentlänge & 1,0 & $0,97-1,03$ & 0,994 \\
\hline Stentdurchmesser & 1,19 & $0,53-2,66$ & 0,676 \\
\hline Proximale Stentlokalisation & 0,63 & $0,31-1,29$ & 0,206 \\
\hline Mehrgefäßerkrankung & 0,64 & $0,31-1,31$ & 0,223 \\
\hline HADS- $A \geq 10$ & 0,21 & $0,05-0,91$ & $0,037 *$ \\
\hline
\end{tabular}

*signifikant auf dem 5\%-Niveau; **signifikant auf dem 1\%-Niveau; ***signifikant auf dem 0,1\%-Niveau 
Tabelle C.2: Cox-Regressionsanalyse mit ausgewählten Confoundern zur Berechnung des kumulativen ereignisfreien Überlebens (MACE) in 5 Jahren Nachbeobachtung bei HADS$\mathrm{A} \geq 10, \mathrm{p}$-Wert für das Gesamtmodell $<0,001$

\begin{tabular}{|c|c|c|c|}
\hline Kovariate & Hazard Ratio & $\begin{array}{c}\text { 95\%-Konfidenz- } \\
\text { intervall }\end{array}$ & p-Wert \\
\hline Geschlecht & 0,97 & $0,51-1,82$ & 0,913 \\
\hline Alter & 1,02 & $1,0-1,05$ & 0,085 \\
\hline Body-Mass-Index & 0,98 & $0,91-1,05$ & 0,536 \\
\hline Hypertonie & 1,16 & $0,59-2,28$ & 0,664 \\
\hline Hypercholesterinämie & 0,61 & $0,36-1,03$ & 0,064 \\
\hline Diabetes mellitus & 0,92 & $0,5-1,68$ & 0,779 \\
\hline Tabakabusus & 2,82 & $1,58-5,03$ & $<0,001 * * *$ \\
\hline Positive Familienanamnese & 1,3 & $0,78-2,16$ & 0,318 \\
\hline Z.n. Myokardinfarkt & 1,76 & $1,06-2,92$ & $0,029 *$ \\
\hline Z.n. Bypass-Operation & 2,13 & $1,06-4,28$ & $0,034^{*}$ \\
\hline Stenttyp (DES) & 1,34 & $0,68-2,63$ & 0,401 \\
\hline Stentlänge & 1,01 & $0,99-1,03$ & 0,477 \\
\hline Stentdurchmesser & 1,25 & $0,67-2,34$ & 0,488 \\
\hline Proximale Stentlokalisation & 0,56 & $0,32-0,98$ & $0,042 *$ \\
\hline Mehrgefäßerkrankung & 0,87 & $0,5-1,51$ & 0,622 \\
\hline HADS- $A \geq 10$ & 0,34 & $0,14-0,8$ & $0,014^{*}$ \\
\hline
\end{tabular}

*signifikant auf dem 5\%-Niveau; **signifikant auf dem 1\%-Niveau; ***signifikant auf dem 0,1\%-Niveau 
Tabelle C.3: Cox-Regressionsanalyse mit ausgewähltem Confounder zur Berechnung der Revaskularisationsrate in 5 Jahren Nachbeobachtung bei HADS-A $\geq 10$, p-Wert für das Gesamtmodell $\mathrm{p}=0,003$

\begin{tabular}{|l|c|c|c|}
\hline Kovariate & Hazard Ratio & $\begin{array}{c}\mathbf{9 5 \%} \text {-Konfidenz- } \\
\text { intervall }\end{array}$ & p-Wert \\
\hline Z.n. Bypass-Operation & 2,26 & $1,25-4,1$ & $0,007 * *$ \\
\hline HADS-A $\geq 10$ & 1,62 & $1,0-2,63$ & 0,051 \\
\hline
\end{tabular}

*signifikant auf dem 5\%-Niveau; **signifikant auf dem 1\%-Niveau; ***signifikant auf dem 0,1\%-Niveau 
Tabelle C.4: Cox-Regressionsanalyse mit ausgewählten Confoundern zur Berechnung der Revaskularisationsrate in 5 Jahren Nachbeobachtung bei HADS-A $\geq 10$, p-Wert für das Gesamtmodell $\mathrm{p}=0,136$

\begin{tabular}{|c|c|c|c|}
\hline Kovariate & Hazard Ratio & $\begin{array}{c}\text { 95\%-Konfidenz- } \\
\text { intervall }\end{array}$ & p-Wert \\
\hline Geschlecht & 0,79 & $0,47-1,35$ & 0,393 \\
\hline Alter & 0,99 & $0,96-1,01$ & 0,419 \\
\hline Body-Mass-Index & 1,00 & $0,95-1,06$ & 0,943 \\
\hline Hypertonie & 1,45 & $0,75-2,78$ & 0,268 \\
\hline Hypercholesterinämie & 0,98 & $0,60-1,59$ & 0,933 \\
\hline Diabetes mellitus & 1,48 & $0,92-2,38$ & 0,108 \\
\hline Tabakabusus & 1,01 & $0,57-1,79$ & 0,968 \\
\hline Positive Familienanamnese & 1,11 & $0,71-1,75$ & 0,637 \\
\hline Z.n. Myokardinfarkt & 0,94 & $0,56-1,55$ & 0,799 \\
\hline Z.n. Bypass-Operation & 2,27 & $1,25-4,11$ & $0,007 * *$ \\
\hline Stenttyp (DES) & 0,79 & $0,42-1,48$ & 0,469 \\
\hline Stentlänge & 1,00 & $0,98-1,02$ & 0,910 \\
\hline Stentdurchmesser & 0,56 & $0,30-1,05$ & 0,071 \\
\hline Proximale Stentlokalisation & 1,16 & $0,74-1,82$ & 0,513 \\
\hline Mehrgefäßerkrankung & 0,94 & $0,58-1,53$ & 0,812 \\
\hline HADS- $A \geq 10$ & 1,62 & $1,00-2,63$ & $0,050^{*}$ \\
\hline
\end{tabular}

*signifikant auf dem 5\%-Niveau; **signifikant auf dem 1\%-Niveau; ***signifikant auf dem 0,1\%-Niveau 
Tabelle C.5: Gruppenvergleich von Patienten mit und ohne HADS-A $\geq 10$

\begin{tabular}{|c|c|c|c|c|}
\hline Kovariate & $\begin{array}{c}\text { Totale Ko- } \\
\text { horte } \\
(n=470)\end{array}$ & $\begin{array}{c}\text { HADS-A }<10 \\
(n=356)\end{array}$ & $\begin{array}{c}\text { HADS-A } \geq 10 \\
(n=106)\end{array}$ & $\begin{array}{l}\text { Gruppen- } \\
\text { vergleich } \\
\text { (p-Wert) }\end{array}$ \\
\hline Geschlecht (männlich) & $76,8 \%$ & $80,6 \%$ & $63,2 \%$ & $<0,001 * * *$ \\
\hline Alter (Jahre) (SD) & $63,7(9,9)$ & $65,1(9,7)$ & $59,2(9,1)$ & $<0,001 * * *$ \\
\hline Body-Mass-Index (SD) & $28(4,0)$ & $27,7(3,9)$ & $28,6(4,2)$ & 0,055 \\
\hline Hypertonie & $80,8 \%$ & $82,1 \%$ & $78,3 \%$ & 0,396 \\
\hline Hypercholesterinämie & $67,6 \%$ & $69,7 \%$ & $65,1 \%$ & 0,404 \\
\hline Diabetes mellitus & $23,6 \%$ & $23,2 \%$ & $27,4 \%$ & 0,438 \\
\hline Tabakabusus & $21,9 \%$ & $19,8 \%$ & $28,3 \%$ & 0,080 \\
\hline Positive Familienanamnese & $38,5 \%$ & $37,7 \%$ & $44,3 \%$ & 0,257 \\
\hline Z.n. Myokardinfarkt & $26,6 \%$ & $27,7 \%$ & $23,6 \%$ & 0,454 \\
\hline Z.n. Bypass-Operation & $12,1 \%$ & $13,5 \%$ & $8,5 \%$ & 0,238 \\
\hline Stenttyp (DES) & $15,5 \%$ & $17,4 \%$ & $10,4 \%$ & 0,319 \\
\hline Stentlänge (mm) (SD) & $19,9(9,9)$ & $20,3(10,0)$ & $18,5(8,6)$ & 0,102 \\
\hline Stentdurchmesser $(\mathrm{mm})(\mathrm{SD})$ & $2,9(0,4)$ & $2,9(0,4)$ & $2,9(0,4)$ & 0,927 \\
\hline Proximale Stentlokalisation & $39,6 \%$ & $37,5 \%$ & $46,2 \%$ & 0,114 \\
\hline Mehrgefäßerkrankung & $51,5 \%$ & $54,5 \%$ & $42,5 \%$ & $0,035^{*}$ \\
\hline HADS-A bei Einschluss (SD) & $6,8(3,7)$ & $5,3(2,4)$ & $12,1(1,9)$ & $<0,001^{* * *}$ \\
\hline Mortalität & $44(9,4 \%)$ & $42(11,8 \%)$ & $2(1,9 \%)$ & $0,002 * *$ \\
\hline MACE & $73(15,5 \%)$ & $66(18,5 \%)$ & $7(6,6 \%)$ & $0,003 * *$ \\
\hline Revaskularisation & $87(18,5 \%)$ & $59(16,6 \%)$ & $28(26,4 \%)$ & $0,028^{*}$ \\
\hline
\end{tabular}

*signifikant auf dem 5\%-Niveau; **signifikant auf dem 1\%-Niveau; ***signifikant auf dem 0,1\%-Niveau 


\section{LITERATURVERZEICHNIS}

Albert CM, Chae CU, Rexrode KM, Manson JE, Kawachi I (2005): Phobic anxiety and risk of coronary heart disease and sudden cardiac death among women. Circulation $\underline{111}, 480-487$

Al-Ruzzeh S, Athanasiou T, Mangoush O, Wray J, Modine T, George S, Amrani M (2005): Predictors of poor mid-term health related quality of life after primary isolated coronary artery bypass grafting surgery. Heart $\underline{91}, 1557-1562$

Barefoot JC, Brummett BH, Helms MJ, Mark DB, Siegler IC, Williams RB (2000): Depressive symptoms and survival of patients with coronary artery disease. Psychosom Med $\underline{62}$, 790-795

Barth J, Schumacher M, Herrmann-Lingen C (2004): Depression as a risk factor for mortality in patients with coronary heart disease: a meta-analysis. Psychosom Med $\underline{66}, 802-813$

Bergvik S, Sorlie T, Wynn R, Sexton H (2010): Psychometric properties of the type D scale (DS14) in Norwegian cardiac patients. Scand J Psychol 51 (4), 334-340

Beutel ME, Wiltink J, Till Y, Wild PS, Münzel T, Ojeda FM, Zeller T, Schnabel RB, Lackner K, Blettner M, Zwiener I, Michal M (2012): Type D personality as a cardiovascular risk marker in the general population: results from the Gutenberg health study. Psychother Psychosom $\underline{81}, 108-117$

Bjelland I, Dahl AA, Haug TT, Neckelmann D (2002): The validity of the Hospital Anxiety and Depression Scale. An updated literature review. J Psychosom Res $\underline{52}$, 69-77

Blumenthal, JA, Thompson LW, Williams RB Jr, Kong Y (1979): Anxiety-proneness and coronary heart disease. J Psychosom Res $\underline{23}, 17-21$

Broek KC van den, Nyklíček I, van der Voort PH, Alings M, Meijer A, Denollet J (2009): Risk of ventricular arrhythmia after implantable defibrillator treatment in anxious type D patients. J Am Coll Cardiol 54, 531-537

Bui E, Cailhol L, Puel J, Birmes P, Schmitt L (2010): Effects of depressive symptoms, anxiety and alexithymia on physical functioning 6 months after percutaneous transluminal coronary angioplasty. Psychother Psychosom $\underline{79}$, 59-60

Carney RM, Rich MW, Freedland KE, Saini J, teVelde A, Simeone C, Clark K (1988): Major depressive disorder predicts cardiac events in patients with coronary artery disease. Psychosom Med $\underline{50}, 627-633$ 
Carney RM, Blumenthal JA, Catellier D, Freedland KE, Berkman LF, Watkins LL, Czajkowski SM, Hayano J, Jaffe AS (2003): Depression as a risk factor for mortality after acute myocardial infarction. Am J Cardiol 92, 1277-1281

Chida Y, Steptoe A (2009): The association of anger and hostility with future coronary heart disease: a meta-analytic review of prospective evidence. J Am Coll Cardiol 53, 936-946

Conraads VM, Denollet J, DeClerck LS, Stevens WJ, Bridts C, Vrints CJ (2006): Type D personality is associated with increased levels of tumour necrosis factor (TNF)-alpha and TNF-alpha receptors in chronic heart failure. Int J Cardiol 113 $\underline{13}$ 34-38

Coyne JC, de Voogd JN (2012): Are we witnessing the decline effect in the Type D personality literature? What can be learned? J Psychosom Res $\underline{73}$, 401-407

Coyne JC, Jaarsma T, Luttik ML, van Sonderen E, van Veldhuisen DJ, Sanderman R (2011): Lack of prognostic value of Type D personality for mortality in a large sample of heart failure patients. Psychosom Med $\underline{73}$, 557-562

Damen NL, Versteeg H, Boersma E, Serruys PW, van Geuns RJ, Denollet J, van Domburg RT, Pedersen SS (2013): Depression is independently associated with 7-year mortality in patients treated with percutaneous coronary intervention: Results from the RESEARCH registry. Int J Cardiol 167 (6), 2496-2501

Denollet J (1993): Biobehavioral research on coronary heart disease: Where is the person? Journal of Behavioral Medicine Vol. 16 (2), 115-141

Denollet J (1998): Personality and coronary heart disease: The type-D scale-16 (DS16). Ann Behav Med 20, 209-215

Denollet J (2000): Type D personality: A potential risk factor refined. Journal of Psychosomatic Research $\underline{49}$, 255-266

Denollet J (2005): DS14: standard assessment of negative affectivity, social inhibition, and Type D personality. Psychosom Med $\underline{67}$, 89-97

Denollet J, Brutsaert DL (1998): Personality, disease severity, and the risk of long-term cardiac events in patients with a decreased ejection fraction after myocardial infarction. Circulation 97, $167-173$ 
Denollet J, Sys SU, Brutsaert DL (1995): Personality and mortality after myocardial infarction. Psychsom Med 57, 582-591

Denollet J, Vaes J, Brutsaert DL (2000): Inadequate response to treatment in coronary heart disease: adverse effects of type D personality and younger age on 5-year prognosis and quality of life. Circulation 102, 630-635

Denollet J, Schiffer AA, Spek V (2010): A general propensity to psychological distress affects cardiovascular outcomes: evidence from research on the type D (distressed) personality profile. Circ Cardiovasc Qual Outcomes 3, 546-557

Denollet J, Pedersen SS, Vrints CJ, Conraads VM (2006a): Usefulness of type D personality in predicting five-year cardiac events above and beyond concurrent symptoms of stress in patients with coronary heart disease. Am J Cardiol 97, 970-973

Denollet J, Strik JJ, Lousberg R, Honig A (2006b): Recognizing increased risk of depressive comorbidity after myocardial infarction: looking for 4 symptoms of anxiety-depression. Psychother Psychsom 75, 346-352

Denollet J, Sys SU, Stoobant N, Rombouts H, Gillebert TC, Brutsaert DL (1996): Personality as independent predictor of long-term mortality in patients with coronary heart disease. Lancet $\underline{347}, 417-421$

Denollet J, Pedersen SS, Ong AT, Erdman RA, Serruys PW, van Domburg RT (2005): Social inhibition modulates the effect of negative emotions on cardiac prognosis following percutaneous coronary intervention in the drug-eluting stent era. Eur Heart J 27, 171-177

Denollet J, Pedersen SS, Daemen J, de Jaegere P, Serruys PW, van Domburg RT (2007): Reduced positive affect (anhedonia) predicts major clinical events following implantation of coronary-artery stents. J Intern Med 263 (2), 203-211

Denollet J, Schiffer AA, Kwaijtaal M, Hooijkaas H, Hendriks EH, Widdershoven JW (2009a): Usefulness of Type D personality and kidney dysfunction as predictors of interpatient variability in immune activation in chronic heart failure. Am J Cardiol 103, 399-404

Denollet J, Maas K, Knottnerus A, Keyzer JJ, Pop VJ (2009b): Anxiety predicted premature all-cause and cardiovascular death in a 10-year follow-up of middle-aged women. J Clin Epidemiol $\underline{62}, 452-456$ 
Denollet J, de Jonge P, Kuyper A, Schene AH, van Melle JP, Ormel J, Honig A (2009c): Depression and Type D personality represent different forms of distress in the Myocardial INfarction and Depression-Intervention Trial (MIND-IT). Psychol Med 39, 749-756

Ebrahim S, Smith GD (1997): Systematic review of randomized controlled trials of multiple risk factor interventions for preventing coronary heart disease. BMJ $\underline{314}$, 1666-1674

Emilien G, Durlach C, Lepola U, Dinan T: Anxiety Disorders: Pathophysiology and Pharmacological Treatment. Birkhauser Verlag, Basel 2002, S. 16

Emons WH, Meijer RR, Denollet J (2007): Negative affectivity and social inhibition in cardiovascular disease: evaluating type-D personality and its assessment using item response theory. J Psychosom Res $\underline{63}, 27-39$

Franco M, Cooper RS, Bilal U, Fuster V (2011): Challenges and Opportunities for Cardiovascular Disease Prevention. The American Journal of Medicine 124 (2), 95-102

Frasure-Smith N, Lesperance F (2008): Depression and anxiety as predictors of 2-year cardiac events in patients with stable coronary artery disease. Arch Gen Psychiatry $\underline{65}, 62-71$

Frasure-Smith N, Lesperance F, Talajic M (1995a): Depression and 18-month prognosis after myocardial infarction. Circulation 91, 999-1005

Frasure-Smith N, Lesperance F, Talajic M (1995b): The impact of negative emotions on prognosis following myocardial infarction: is it more than depression? Health Psychol $\underline{14}$, 388-398

Frasure-Smith N, Lesperance F, Gravel G, Masson A, Juneau M, Talajic M, Bourassa MG (2000): Social support, depression, and mortality during the first year after myocardial infarction. Circulation 101, 1919-1924

Grace SL, Abbey SE, Kapral MK, Fang J, Nolan RP, Stewart DE (2005): Effect of depression on five-year mortality after an acute coronary syndrome. Am J Cardiol 무, 1179-1185

Grande G, Romppel M, Barth J (2012): Association between type D personality and prognosis in patients with cardiovascular diseases: a systematic review and meta-analysis. Ann Behav Med $\underline{43}, 299-310$

Grande G, Romppel M, Vesper JM, Schubmann R, Glaesmer H, Hermann-Lingen C (2011): Type D personality and all-cause mortality in cardiac patients. Data from a German cohort study. Psychosom Med $\underline{73}$, 548-556 
Grande G, Jordan J, Kümmel M, Struwe C, Schubmann R, Schulze F, Unterberg C, von Känel R, Kudielka BM, Fischer J, Herrmann-Lingen C (2004): Evaluation der deutschen TypD Skala (DS14) und Prävalenz der Typ-D-Persönlichkeit bei kardiologischen und psychosomatischen Patienten sowie Gesunden. Psychother Psychosom Med Psychol 54, 413422

Grüntzig AR, Senning A, Siegenthaler WE (1979): Nonoperative dilatation of coronary-artery stenosis: percutaneous transluminal coronary angioplasty. N Engl J Med 301 (2), 61-68

Habra ME, Linden W, Anderson JC, Weinberg J (2003): Type D personality is related to cardiovascular and neuroendocrine reactivity to acute stress. J Psychosom Res $\underline{55}$, 235-245

Haines AP, Imeson JD, Meade TW (1987): Phobic anxiety and ischaemic heart disease. $\mathrm{Br}$ Med J (Clin Res Ed) 295, 297-299

Herrmann C (1997): International experiences with the Hospital Anxiety and Depression Scale. A review of validation data and clinical results. J Psychosom Res $\underline{42}, 17-41$

Herrmann C, Buss U, Snaith RP: HADS-D - Hospital Anxiety and Depression Scale - Deutsche Version: Ein Fragebogen zur Erfassung von Angst und Depressivität in der somatischen Medizin. Verlag Hans Huber, Bern 1995

Herrmann C, Brand-Driehorst S, Buss U, Rüger U (2000): Effects of anxiety and depression on 5-year mortality in 5057 patients referred for exercise testing. J Psychosom Res $\underline{48}, 455-$ 462

Herrmann C, Brand-Driehorst S, Kaminsky B, Leibing E, Staats H, Rüger U (1998): Diagnostic groups and depressed mood as predictors of 22-month mortality in medical inpatients. Psychosom Med 0ㅜ, 570-577

Herrmann-Lingen C, Buss U: Angst und Depressivität im Verlauf der koronaren Herzkrankheit. Reihe: Statuskonferenz Psychokardiologie. Band 5 Verlag VAS, Frankfurt 2002

Herrmann-Lingen C, Binder L, Klinge M, Sander J, Schenker W, Beyermann B, von Lewinski D, Pieske B (2003): High plasma levels of N-terminal pro-atrial natriuretic peptide associated with low anxiety in severe heart failure. Psychosom Med $\underline{65}$, 517-22

Huxley R, Barzi F, Woodward M (2006): Excess risk of fatal coronary heart disease associated with diabetes in men and women: meta-analysis of 37 prospective cohort studies. BMJ $\underline{14}$ (332), 73-78 
Irvine J, Basinski A, Baker B, Jandciu S, Paquette M, Cairns J, Connolly S, Roberts R, Gent M, Dorian P (1999): Depression and risk of sudden cardiac death after acute myocardial infarction: testing for the confounding effects of fatigue. Psychosom Med $\underline{61}, 729-737$

Kaptein KI, de Jonge P, van den Brink RH, Korf J (2006): Course of depressive symptoms after myocardial infarction and cardiac prognosis: a latent class analysis. Psychosom Med $\underline{68}$, $662-668$

Kawachi I, Colditz GA, Ascherio A, Rimm EB, Giovannucci E, Stampfer MJ, Willett WC (1994a): Prospective study of phobic anxiety and risk of coronary heart disease in men. Circulation $\underline{89}, 1992-1997$

Kawachi I, Sparrow D, Vokonas PS, Weiss ST (1994b): Symptoms of anxiety and risk of coronary heart disease. The Normative Aging Study. Circulation 90, 2225-2229

Kornerup H, Zwisler AD, Prescott E (2011): No association between anxiety and depression and adverse clinical outcome among patients with cardiovascular disease: findings from the DANREHAB trial. J Psychosom Res $\underline{71}$, 207-214

Kupper N, Gidron Y, Winter J, Denollet J (2009): Association between type D personality, depression, and oxidative stress in patients with chronic heart failure. Psychosom Med $\underline{71}$, 973-980

Ladwig KH, Lederbogen F, Albus C, Angermann C, Borggrefe M, Fischer D, Fritzsche K, Haass M, Jordan J, Jünger J, Kindermann I, Köllner V, Kuhn B, Scherer M, Seyfarth M, Völler H, Waller C, Herrmann-Lingen C (2013): Positionspapier zur Bedeutung psychosozialer Faktoren in der Kardiologie. Kardiologe $\underline{7}, 7-27$

Lane D, Carroll D, Ring C, Beevers DG, Lip GY (2001): Mortality and quality of life 12 months after myocardial infarction: effects of depression and anxiety. Psychosom Med $\underline{63}$, $221-230$

Lesperance F, Frasure-Smith N, Talajic M, Bourassa MG (2002): Five-year risk of cardiac mortality in relation to initial severity and one-year changes in depression symptoms after myocardial infarction. Circulation $\underline{105}, 1049-1053$

Link N, Tanner M (2001): Coronary artery disease: Part 1. Epidemiology and diagnosis. Western Journal of Medicine 174, 257-261 
Martens EJ, Mols F, Burg MM, Denollet J (2010): Type D personality predicts clinical events after myocardial infarction, above and beyond disease severity and depression. J Clin Psychiatry $\underline{71}, 778-783$

Mayou RA, Gill D, Thompson DR, Day A, Hicks N, Volmink J, Neil A (2000): Depression and anxiety as predictors of outcome after myocardial infarction. Psychosom Med $\underline{62}, 212-$ 219

Meijer A, Conradi HJ, Bos EH, Thombs BD, van Melle JP, de Jonge P (2011): Prognostic association of depression following myocardial infarction with mortality and cardiovascular events: a meta-analysis of 25 years of research. Gen Hosp Psychiatry $\underline{33}$, 203-216

Melle JP van, de Jonge P, Spijkerman TA, Tijssen JG, Ormel J, van Veldhuisen DJ, van den Brink RH, van den Berg MP (2004): Prognostic association of depression following myocardial infarction with mortality and cardiovascular events: a meta-analysis. Psychosom Med $\underline{66}$, 814-22

Meyer T, Buss U, Herrmann-Lingen C (2010): The role of cardiac disease severity in the predictive value of anxiety for all-cause mortality. Psychosom Med $\underline{72}, 9-15$

Molloy GJ, Perkins-Porras L, Strike PC, Steptoe A (2008): Type-D personality and cortisol in survivors of acute coronary syndrome. Psychosom Med $\underline{70}, 863-868$

Mols F, Martens EJ, Denollet J (2010): Type D personality and depressive symptoms are independent predictors of impaired health status following acute myocardial infarction. Heart 96, 30-35

Moser DK, Dracup K (1996): Is anxiety early after myocardial infarction associated with subsequent ischemic and arrhythmic events? Psychosom Med $\underline{58}, 395-401$

Moser DK, Riegel B, McKinley S, Doering LV, An K, Sheahan S (2007): Impact of anxiety and perceived control on in-hospital complications after acute myocardial infarction. Psychosom Med 69, 10-16

Moser DK, McKinley S, Riegel B, Doering LV, Meischke H, Pelter M, Davidson P, Baker H, Dracup K (2011): Relationship of persistent symptoms of anxiety to morbidity and mortality outcomes in patients with coronary heart disease. Psychosom Med $\underline{73}$, 803-809

Müller-Nordhorn J, Willich SN (2008): Coronary Heart Disease. International Encyclopedia of Public Health, 16-25 
Mykletun A, Bjerkeset O, Dewey M, Prince M, Overland S, Stewart R (2007): Anxiety, depression, and cause-specific mortality: The HUNT study. Psychosom Med $\underline{69}, 323-331$

Nakamura S, Kato K, Yoshida A, Fukuma N, Okumura Y, Ito H, Mizuno K (2013): Prognostic value of depression, anxiety, and anger in hospitalized cardiovascular disease patients for predicting adverse cardiac outcomes. Am J Cardiol 2013 111, 1432-1436

Nicholson A, Kuper H, Hemingway H (2006): Depression as an aetiologic and prognostic factor in coronary heart disease: a meta-analysis of 6362 events among 146538 participants in 54 observational studies. Eur Heart J 27, 2763-2774

Ormel J, Rosmalen J, Farmer A (2004): Neuroticism: a non-informative marker of vulnerability to psychopathology. Soc Psychiatry Psychiatr Epidemiol 39, 906-912

Pajak A, Jankowski P, Kotseva K, Heidrich J, de Smedt D, De Bacquer D (2013): EUROASPIRE Study Group. Depression, anxiety, and risk factor control in patients after hospitalization for coronary heart disease: the EUROASPIRE III Study. Eur J Prev Cardiol $\underline{20}, 331-340$

Parakh K, Thombs BD, Fauerbach JA, Bush DE, Ziegelstein RC (2008): Effect of depression on late (8 years) mortality after myocardial infarction. Am J Cardiol 101, 602-606

Pedersen SS, van den Broek KC, Erdman RA, Jordaens L, Theuns DA (2010): Preimplantation implantable cardioverter defibrillator concerns and Type D personality increase the risk of mortality in patients with an implantable cardioverter defibrillator. Europace $\underline{12}$, $1446-1452$

Pedersen SS, Denollet J, de Jonge P, Simsek C, Serruys PW, van Domburg RT (2009): Brief depression screening with the PHQ-2 associated with prognosis following percutaneous coronary intervention with paclitaxel-eluting stenting. J Gen Intern Med. 24 (9), 1037-1042

Pedersen SS, Denollet J, Spindler H, Ong ATL, Serruys PW, Erdman RAM, van Domburg RT (2006): Anxiety enhances the detrimental effect of depressive symptoms on health status following percutaneous coronary intervention. J Psychosom Res $\underline{61}$, 783-789

Pedersen SS, Denollet J, Daemen J, van de Sande M, de Jaegere PT, Serruys PW, Erdman RA, van Domburg RT (2007a): Fatigue, depressive symptoms, and hopelessness as predictors of adverse clinical events following percutaneous coronary intervention with paclitaxeleluting stents. J Psychosom Res $\underline{62}, 455-461$ 
Pedersen SS, Denollet J, Ong AT, Sonnenschein K, Erdman RA, Serruys PW, van Domburg RT (2007b): Adverse clinical events in patients treated with sirolimus-eluting stents: the impact of Type D personality. Eur J Cardiovasc Prev Rehabil 14, 135-140

Pedersen SS, Lemos PA, van Vooren PR, Liu TK, Daemen J, Erdman RA, Smits PC, Serruys PW, van Domburg RT (2004): Type D personality predicts death or myocardial infarction after bare metal stent or sirolimus-eluting stent implantation: a Rapamycin-Eluting Stent Evaluated at Rotterdam Cardiology Hospital (RESEARCH) registry substudy. J Am Coll Cardiol 44, 997-1001

Pelle AJ, Pedersen SS, Schiffer AA, Szabó BM, Widdershoven JW, Denollet J (2010): Psychological distress and mortality in systolic heart failure. Circ Heart Fail $\underline{3}$, 261-267

Perk J, De Backer G, Gohlke H, Graham I, Reiner Z, Verschuren WM, Albus C, Benlian P, Boysen G, Cifkova R, Deaton C, Ebrahim S, Fisher M, Germano G, Hobbs R, Hoes A, Karadeniz S, Mezzani A, Prescott E, Ryden L, Scherer M, Syvänne M, Op Reimer WJ, Vrints C, Wood D, Zamorano JL, Zannad F; European Association for Cardiovascular Prevention \& Rehabilitation (EACPR) (2012): European guidelines on cardiovascular disease prevention in clinical practice (version 2012): the fifth joint task force of the European society of cardiology and other societies on cardiovascular disease prevention in clinical practice (constituted by representatives of nine societies and by invited experts). Int J Behav Med 19, 403-488

Poston WS, Haddock CK, Conard MW, Jones P, Spertus J (2003): Assessing depression in the cardiac patient. When is the appropriate time to assess depression in the patient undergoing coronary revascularization? Behav Modif 27, 26-36

Roest AM, Martens EJ, de Jonge P, Denollet J (2010a): Anxiety and risk of incident coronary heart disease: a meta-analysis. J Am Coll Cardiol $\underline{56}$, 38-46

Roest AM, Martens EJ, Denollet J, de Jonge P (2010b): Prognostic association of anxiety post myocardial infarction with mortality and new cardiac events: a meta-analysis. Psychosom Med 72, 563-569

Romppel M, Herrmann-Lingen C, Vesper JM, Grande G (2012): Six year stability of Type-D personality in a German cohort of cardiac patients. J Psychosom Res Feb $\underline{72}$, 136-141

Rosenman RH, Brand RJ, Jenkins CD, Friedman M, Straus R, Wurm M (1975): Coronary heart disease in the Western Collaborative Group Study: final follow-up experience of $81 / 2$ years. JAMA $\underline{233}, 872-877$ 
Rothenbacher D, Hahmann H, Wüsten B, Koenig W, Brenner H (2007): Symptoms of anxiety and depression in patients with stable coronary heart disease: prognostic value and consideration of pathogenetic links. Eur J Cardiovasc Prev Rehabil 14, 547-554

Rozanski A, Blumenthal JA, Davidson KW, Saab PG, Kubzansky L (2005): The epidemiology, pathophysiology, and management of psychosocial risk factors in cardiac practice: the emerging field of behavioral cardiology. J Am Coll Cardiol 45, 637-651

Rutledge T, Reis VA, Linke SE, Greenberg BH, Mills PJ (2006): Depression in heart failure a meta-analytic review of prevalence, intervention effects, and associations with clinical outcomes. J Am Coll Cardiol 쏘, 1527-1537

Schiffer AA, Pedersen SS, Widdershoven JW, Denollet J (2008): Type D personality and depressive symptoms are independent predictors of impaired health status in chronic heart failure. Eur J Heart Fail 10, 802-810

Schmidt MM, Quadros AS, Abelin AP, Minozzo EL, Wottrich SH, Kunert HZ, Vigueras ES, Ruschel PP, Sarmento-Leite R, Gottschall CA (2011): Psychological characteristics of patients undergoing percutaneous coronary interventions. Arq Bras Cardiol 97, 331-337

Schömig A, Mehilli J, de Waha A, Seyfarth M, Pache J, Kastrati A (2008): A meta-analysis of 17 randomized trials of a percutaneous coronary intervention-based strategy in patients with stable coronary artery disease. J Am Coll Cardiol 52, 894-904

Secretariat PSC (2008): Review: lower total cholesterol is associated with reduced risk of death from ischaemic heart disease but not stroke. Evid Based Med 13 (3), 87

Shen BJ, Avivi YE, Todaro JF, Spiro A 3rd, Laurenceau JP, Ward KD, Niaura R (2008): Anxiety characteristics independently and prospectively predict myocardial infarction in men the unique contribution of anxiety among psychologic factors. J Am Coll Cardiol 51, 113-119 Shibeshi WA, Young-Xu Y, Blatt CM (2007): Anxiety worsens prognosis in patients with coronary artery disease. J Am Coll Cardiol 22, 2021-2027

Smith TW (2011): Toward a more systematic, cumulative, and applicable science of personality and health: lessons from type D personality. Psychosom Med $\underline{73}, 528-532$

Spinhoven P, Ormel J, Sloekers PP, Kempen GI, Speckens AE, van Hemert AM (1997): A validation study of the Hospital Anxiety and Depression Scale (HADS) in different groups of Dutch subjects. Psychol Med 27, 363-370 
Stewart RA, North FM, West TM, Sharples KJ, Simes RJ, Colquhoun DM, White HD, Tonkin AM (2003): Depression and cardiovascular morbidity and mortality: cause or consequence? Eur Heart J 24, 2027-2037

Strik JJ, Denollet J, Lousberg R, Honig A (2003): Comparing symptoms of depression and anxiety as predictors of cardiac events and increased health care consumption after myocardial infarction. J Am Coll Cardiol 19, 1801-1807

Sullivan MD, LaCroix AZ, Spertus JA, Hecht J (2000): Five-year prospective study of the effects of anxiety and depression in patients with coronary artery disease. Am J Cardiol $\underline{86}$, $1135-1138$

Sullivan MD, LaCroix AZ, Spertus JA, Hecht J, Russo J (2003): Depression predicts revascularization procedures for 5 years after coronary angiography. Psychosom Med $\underline{65}$, 229-36

Trikalinos TA, Alsheikh-Ali AA, Tatsioni A, Nallamothu BK, Kent DM (2009): Percutaneous coronary interventions for non-acute coronary artery disease: a quantitative 20-year synopsis and a network meta-analysis. Lancet $\underline{373}, 911-918$

Tunstall-Pedoe H, Kuulasmaa K, Mähönen M, Tolonen H, Ruokokoski E, Amouvel P für das WHO MONICA Projekt (1999): Contribution of trends in survival and coronary-event rates to changes in coronary heart disease mortality: 10-year results from 37 WHO MONICA Project populations. The Lancet 353 (9164), 1547-1557

Volz A, Schmid JP, Zwahlen M, Kohls S, Saner H, Barth J (2011): Predictors of readmission and health related quality of life in patients with chronic heart failure: a comparison of different psychosocial aspects. J Behav Med $\underline{34}$, 13-22

Voogd JN de, Wempe JB, Postema K, von Sonderen E, Ranchor AV, Coyne JC, Sanderman R (2009): More evidence that depressive symptoms predict mortality in COPD patients: Is type D personality an alternative explanation? Ann Behav Med $\underline{38}$, 86-93

Vreeburg SA, Hoogendijk WJ, van Pelt J, Derijk RH, Verhagen JC, van Dyck R, Smit JH, Zitman FG, Penninx BW (2009): Major depressive disorder and hypothalamic-pituitaryadrenal axis activity: results from a large cohort study. Arch Gen Psychiatry $\underline{66}, 617-626$

Welin C, Lappas G, Wilhelmsen L (2000): Independent importance of psychosocial factors for prognosis after myocardial infarction. J Intern Med 247, 629-639 
Whitehead DL, Perkins-Porras L, Strike PC, Magid K, Steptoe A (2007): Cortisol awakening response is elevated in acute coronary syndrome patients with type-D personality. J Psychosom Res $\underline{62}, 419-425$

Williams L, O’Connor R, Howard S, Hughes BM, Johnston DW, Hay JL, O’Connor DB, Lewis A, Ferguson E, Sheehy N, Grealy MA, O’Carroll RE (2008): Type-D personality mechanisms of effect: The role of health-related behavior and social support. J Psychosom Res $\underline{64(1)}, 63-69$

Zigmond AS, Snaith RP (1983): The hospital anxiety and depression scale. Acta Psychiatr Scand 67, 361-370 


\section{Danksagung}

Mein besonderer Dank gilt Herrn Dr. med. Helmut Lange, der die vorliegende Studie ermöglichte und mich stets bei der Datenerhebung im Herzkatheterlabor des Klinikums Links der Weser unterstützte. In gleicher Weise möchte ich mich bei Mohammad N. Pasalary bedanken, der mit der Erfassung der Fragebögen zum wesentlichen Fortschritt der Arbeit beigetragen hat.

Ganz herzlich möchte ich mich bei Herrn Prof. Dr. med. C. Herrmann-Lingen bedanken, der mich als Doktorvater über den gesamten Zeitraum großartig betreute und mich stets bei statistischen Analysen unterstützte. Herrn Prof. Dr. mult. Thomas Meyer gebührt besonderer Dank für die Hilfe bei der Berechnung, der Interpretation und der Veröffentlichung der Daten.

Ebenfalls großer Dank geht an das gesamte Team des Herzkatheterlabors des Klinikums Links Der Weser für die Unterstützung beim Erheben der Daten.

Zuletzt möchte ich mich bei allen Mitarbeitern der Klinik für Psychosomatische Medizin und Psychotherapie für die angenehme Arbeitsatmosphäre und den sehr freundlichen Umgang bedanken. 


\section{VERÖFFENTLICHUNGEN MIT BEZUG ZUR DISSERTATIONSSCHRIFT}

Aus meinem Dissertationsprojekt sind folgende Originalpublikationen in Peer-begutachteten Zeitschriften erschienen:

Meyer T, Hussein S, Lange HW, Herrmann-Lingen C (2014): Transient impact of baseline depression on mortality in patients with stable coronary heart disease during long-term follow-up. Clin Res Cardiol 103 (5), 389-395

Meyer T, Hussein S, Lange HW, Herrmann-Lingen C (2014): Type D personality is unrelated to major adverse cardiovascular events in patients with coronary artery disease treated by intracoronary stenting. Ann Behav Med 48 (2), 156-162

Meyer T, Hussein S, Lange HW, Herrmann-Lingen C (2013): Anxiety is associated with a reduction in both mortality and major adverse cardiovascular events five years after coronary stenting. Eur J Prev Cardiol. [Epub ahead of print] 